\title{
More hedging instruments may destabilize markets
}

\author{
William Brock ${ }^{a}$ \\ Cars Hommes ${ }^{b *}$ \\ Florian Wagener ${ }^{b}$
}

September 2006

\begin{abstract}
This paper formalizes the idea that more hedging instruments may destabilize markets when traders are heterogeneous and adapt their behavior according to experience based reinforcement learning. We investigate three different economic settings, a simple mean-variance asset pricing model, a general equilibrium two-period overlapping generations model with heterogeneous expectations and a noisy rational expectations asset pricing model with heterogeneous information signals. In each setting the introduction of additional Arrow securities can destabilize the market, causing a bifurcation of the steady state to multiple steady states, periodic orbits or even chaotic fluctuations.
\end{abstract}

keywords: asset pricing, hedging, reinforcement learning, nonlinear dynamics, bifurcations

Acknowledgments. Earlier versions of this paper have been presented at the SCE-conference on Computational Economics and Finance, Amsterdam, July 8-10, 2004, the workshop "Volatility of financial markets: theoretical models, forecasting and trading", at the Lorentz Center Leiden, October 18-29, 2004 and at the workshop on "Complexity and Randomness in Economic Dynamical Systems", Bielefeld, March 17-19, 2005. Stimulating discussions and helpful comments from participants are gratefully acknowledged. This research has been supported by the Netherlands Organization for Scientific Research (NWO), the NSF, the Vilas Trust and by a EU STREP-grant "Complex Markets".

${ }^{a}$ Department of Economics, University of Wisconsin, 1180 Observatory Drive, Madison, WI, USA.Email: wbrock@ssc.wisc.edu.

${ }^{b}$ CeNDEF, Department of Quantitative Economics, University of Amsterdam, Roetersstraat 11, 1018WB Amsterdam (the Netherlands). Email: C.H.Hommes@uva.nl (Hommes),

F.O.O.Wagener@uva.nl (Wagener).

${ }^{*}$ Corresponding author. 


\section{Contents}

1 Introduction 1

2 Asset pricing model 5

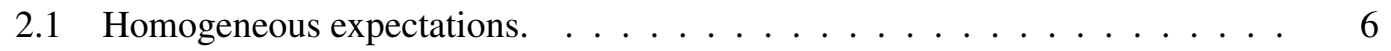

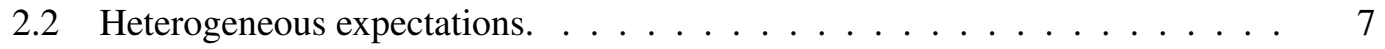

2.3 Adding Arrow securities. . . . . . . . . . . . . . . . . . . . . 11

2.4 Examples. . . . . . . . . . . . . . . . . . . . . . . . . 14

$3 \quad$ Two period overlapping generations model $\quad 20$

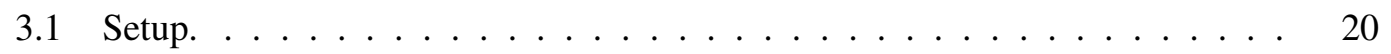

3.2 Homogeneous agents. . . . . . . . . . . . . . . . . . 21

3.3 Heterogeneous agents. . . . . . . . . . . . . . 23

3.4 Dynamics close to a steady state equilibrium. . . . . . . . . . . . . . . . 24

4 Information dynamics $\quad 33$

4.1 Equilibrium price dynamics. . . . . . . . . . . . . . . . . . . . 34

4.2 The model with a short lived asset and reinforcement learning. . . . . . . . . 39

4.3 Adding more Arrow securities. . . . . . . . . . . . . . . . . . . . . . . 41

5 Concluding Remarks 43

A Proof of the lemma

B Proof of bifurcations in 2-type example

C Proof of the existence of an equilibrium price $\quad 56$

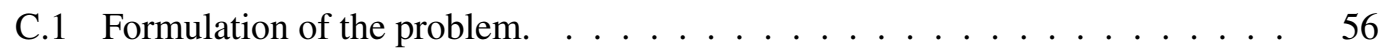

C.2 Reformulation of the problem. . . . . . . . . . . . . . . 57

C.3 Nonvanishing demands. . . . . . . . . . . . . . . . . . . . . . . . . . . . 59

C.4 The homotopy argument. . . . . . . . . . . . . . . . . . . . . 62 
"Our fundamental risks will thus be insured against, hedged, diversified, making for a safer world. By lightening the burden of risk, a new democratic finance will encourage all of us to be more venturesome, more inspired in our activities.”, Robert J. Shiller, The New Financial Order: Risk in the 21st Century, Princeton University Press, 2003.

\section{Introduction}

Robert J. Shiller (2003) advocates an expansion of the number of risk hedging instruments. We support his argument. But there are some issues of reinforcement learning, price adjustment, potential instability and excess volatility in a world that has more hedging instruments which we wish to discuss in this paper. Rajan (2005) has recently raised similar concerns arguing that due to revolutionary changes in the financial sector markets may be more exposed to financial turmoil. In particular, Rajan notes an explosive growth of investment instruments and global investment opportunities as well as a different type of investment management, moving away from traditional banks to mutual funds, insurance companies, pension funds and investment firms. Among these investment managers, incentives based on relative performance as measured by realized returns seem to play an increasingly important role. Before we begin, we emphasize that we do not dispute the potential welfare increasing effects of adding more risk hedging instruments. Our concern is with the impact of adding more risk hedging instruments upon adjustment paths towards new equilibria when a new risk hedging instrument is added.

This paper formalizes the idea that more hedging instruments or derivative securities may destabilize a market when traders are heterogeneous and learn from experience based on realized returns. Here is a sketch of the idea. Consider a heterogeneous agent intertemporal asset market where risk averse agents are learning the structure of asset prices in the economy by using, for example, different prediction strategies of future asset prices under some kind of reinforcement or evolutionary learning, for instance as in Brock and Hommes (1997). Let there be $S$ states of the world and a finite number of contingent claims or risk hedging instruments available for $n<S$ states of the world. We model the risk hedging instruments as "Arrow" securities for state $s, 1 \leq s \leq n<S$, each paying 1 if state $s$ occurs and 0 otherwise. Elementary Arrow securities are used here as a convenient analytical device, but may be viewed as proxies for more realistic financial securities such as futures or derivative securities. Now suppose a new risk hedging instrument, that is, a new Arrow security, is added for state $n+1<S$. Then, since agents are risk averse, and since they can use the new Arrow security to hedge out "extra" risk, they will now tend to place bigger positions on the market. Thus if an agent uses a predictor or purchases a "signal" and that forecasting tool turns out to be on the "right side" of the market, it will return a larger profit (because a larger position has been placed on the market), therefore it will receive a stronger reinforcement. Under reinforcement learning this implies that more 
individuals will switch to using that particular forecasting tool. This, in turn, implies that the learning system is now more likely to "overshoot", i.e. to become unstable. This intuitive idea will be formalized in three different model settings, and for each we will show that adding more hedging instruments may destabilize market dynamics. Our main tools of analysis stem from nonlinear dynamics and bifurcation theory as treated for instance in Guckenheimer and Holmes (1983), Grandmont (1988), Arrowsmith and Place (1994), Kuznetsov (1995) and Medio and Lines (2001). Early economic applications of nonlinear dynamics have been discussed extensively in e.g. Grandmont $(1985,1986)$ and Boldrin and Woodford (1990).

Our first model is a simple mean-variance two period trading framework based upon Brock and Hommes (1998), which we use to expose the potential increased instability of reinforcement learning in a minimalistic setting. In the model several prediction strategies are available to the agents, who base their choice upon measures of past performance. There is a large number of agents, and their behavior is described by a discrete choice model which gives simple analytical forms for the choice probabilities; see e.g. Anderson, de Palma and Thisse (1993) for many other economic applications. We show that the critical value of a bifurcation parameter, marking the onset of instability, is "smaller" when more Arrow securities are added. While this framework is overly simple and is partial equilibrium, it has enough structure to expose the role of a key lemma about nested positive definite matrices that enables us to show that "larger" positions will be taken when there are more Arrow securities available to "hedge out" risks. The larger position turns out to be enough to show that bifurcation towards instability occurs "earlier" when there are more Arrow securities. We provide examples where adding more Arrow securities leads to multiple steady states through saddlenode bifurcations and to an unstable steady state through a Hopf bifurcation leading to periodic and eventually even chaotic asset price fluctuations.

The second model is a two period general equilibrium overlapping generations (OG) model. The OG-model has become a benchmark model in economic dynamics and the possibility of complicated dynamical behavior has been pointed out in the pioneering work by Benhabib and Day (1982) and Grandmont (1985). A novel aspect of our model is that agents have heterogeneous expectations about next period's price of a risky asset; see Brock and DeFontnouvelle (2000) for an earlier OG-model with heterogeneous beliefs. It turns out that a very similar result can be established in this more complicated two-period OG-model. That is to say, a bifurcation in the dynamics of the reinforcement learning system occurs "earlier" if more Arrow securities are added. A quadratic approximation of the OG-model around the steady state leads in fact to the same dynamics as in the first, mean-variance asset pricing model. In particular, local bifurcations of the steady states in both frameworks coincide.

The third model is a noisy rational expectations asset pricing model in which agents receive information signals about stochastic future dividends, following the pioneering work of Grossman and Stiglitz (1980). The dividend of the asset consists of a sum of $S$ independent random variables and each "Arrow security", $i$, corresponds to 
a tradeable claim on the $i$-th random variable that makes up the sum of $S$ independent random variables. Agents have rational expectations about future asset prices in this model, but they have heterogeneous information signals about future dividends. DeFontnouvelle (2000) and Goldbaum $(2005,2006)$ have also studied this type of model with reinforcement learning. The novel aspect in our paper is how Arrow securities affect market stability. We show that, when better information is more costly, again, for much the same reason, bifurcation occurs earlier in the reinforcement learning system when more Arrow securities are added. We provide an example where adding more Arrow securities destabilizes the system through a period doubling bifurcation.

Our work exposes a tension within incomplete markets general equilibrium systems where learning takes place. On the one hand adding Arrow securities helps to remove heterogeneity in beliefs about different states of the world. For example, if there is an Arrow security for every state of the world, then equilibration in the pricing of these objects is a powerful force towards homogenization of beliefs. But on the other hand, if agents in the model are using different strategies (e.g. different predictors of future prices or different signals about future dividends) and the strategies are chosen according to past performances, then increasing the number of Arrow securities may tend to increase the potential for instability of the learning system. This is so because increasing the number of risk hedging instruments enables an agent using a particular strategy to take a larger position on the market while bearing the same amount of risk. Thus if that strategy ends up performing relatively better than others, profits for that predictor will tend to be larger and hence it will be reinforced more in a reinforcement learning system. For an extensive survey on incomplete markets see Magill and Quinzii (1994) and references therein. General surveys on learning and bounded rationality in economics include Evans and Honkapohja (2001), Grandmont (1998), Guesnerie (2002), Kurz (1994), Marimon (1997) and Sargent (1993). Our work is also related to the work on evolutionary selection and learning in complete and incomplete markets by, e.g., Blume and Easley (1992, 2006), Araújo and Sandroni (1999), Sandroni (2000, 2005), Amir et al. (2005), Evstigneev et al. (2002), and Hens and SchenkHoppé (2005). None of these papers however investigates how the addition of Arrow securities affects market stability.

Before we get into details of the paper we must say something about the potential generality of our result that more hedging instruments may destabilize markets. Obviously this kind of result can not appear in a complete markets intertemporal general equilibrium model with no learning of any kind. In this paper we show the result to be valid in three different settings, a mean-variance asset pricing framework, a twoperiod OG general equilibrium framework and an information dynamics framework. We believe it might appear in generalizations of economic learning systems, such as Arifovic (1994), Arthur (1994), Bullard (1994), Bullard and Duffy (1998,2001), Marcet and Nicolini (2003), Schönhofer (1999) and Woodford (1990). Heterogeneous expectations play an increasingly important role in economics and finance, and models with heterogeneous learning agents have been studied recently by e.g. Arthur et 
al. (1997), Branch (2004), Branch and Evans (2005, 2006), Brock and DeFontnouvelle (2000), Brock et. al. (2005), Chiarella and He (2003), DeGrauwe and Grimaldi (2005), DeLong et al. (1990ab), Gaunersdorfer (2000), Kirman (1993), LeBaron et al. (1999), Scheinkman and Xiong (2004) and Sethi and Franke (1995); see LeBaron (2006) and Hommes (2006) for two up to date reviews of learning in a heterogeneous agent framework, Kirman (2006) for a recent discussion of the role of heterogeneity and Barberis and Thaler (2003) for a survey of related work in behavioral finance. Information dynamic financial models with heterogeneous information signals have been studied recently by DeFontnouvelle (2000) and Goldbaum $(2005,2006)$.

From a methodological viewpoint we note that, in these different economic settings, instability can arise through a Hopf, a saddle-node or a period doubling bifurcation. Each of these three generic co-dimension one bifurcations toward instability can arise when adding more Arrow securities. The examples discussed here in three different economic settings thus provide all generic possibilities from a mathematical viewpoint. For a mathematical treatment of generic co-dimension one bifurcations we refer to Guckenheimer and Holmes (1983), Grandmont (1988), and Kuznetsov (1995).

This paper is organized as follows. Section 2 presents a simple two period mean variance heterogeneous agent asset pricing model. This is a minimalist model for determination of equilibrium asset prices, but it is enough to formalize the idea that adding Arrow securities causes increased reinforcement of past successful predictors and may generate instability. Section 3 develops a general equilibrium overlapping generations asset pricing model with heterogeneous beliefs. Although this model is not as analytically tractable as the first model, it is still tractable enough that we are able to introduce reinforcement learning. We obtain a very similar result as we obtain in the first model. In fact, a linear-quadratic approximation of the OG-model around any homogeneous expectations steady state equilibrium yields the same dynamics, up to higher order terms, as the mean-variance asset pricing framework. Section 4 introduces the third framework, a noisy rational expectations model, where dividends are given by a sum of $S$ independent random variables. The object that plays the role of an Arrow security is now a claim on the $i$ th part of the dividend random variable. Again we are able to show that under reinforcement learning, addition of another claim to the existing set of claims causes bifurcation to occur "earlier." It is of interest to note that a key lemma on the behavior of nested positive definite matrices plays an important role in the proofs of the result for all three models even though the models are quite different. This is one reason why we think there is hope that our kind of result can be generalized to more realistic settings such as models with longer horizons. The paper closes with a summary, conclusions, and suggestions for future research. Three Appendices provide a proof of the key matrix theoretic lemma, a proof of the bifurcation structure of a two-type example of the mean-variance asset pricing model as well as a self contained proof of existence of an equilibrium price vector in the OG-model. The latter is included not only to make the paper self contained but also to deal with some existence issues that seem difficult to find in the literature. 


\section{Asset pricing model}

In this section we extend the asset pricing model with heterogeneous beliefs of Brock and Hommes $(1997,1998)$ by adding contingent claims or Arrow securities. There are $S$ possible states of the world in period $t+1$. They occur with probabilities $\alpha_{s}$, $1 \leq s \leq S$, which are independent of time and which are common knowledge. Agents can buy risk free bonds and two types of risky assets, stocks and Arrow securities. Bonds are bought at a fixed price 1 and pay $R>1$ in the next period. Stocks are bought at a market price $p_{t}^{0}$ at time $t$, and at time $t+1$ in state of the world $s$ they pay an amount

$$
q_{t+1}^{s}=p_{t+1}^{0}+y^{s}
$$

which is the sum of the new market price $p_{t+1}^{0}$ and a dividend $y^{s}$ that depends on the state of the world. Finally Arrow securities for state $i$ are bought at a market price $p_{t}^{i}$ and pay a quantity $\delta_{i}^{s}$, which is 1 if $s=i$ and 0 otherwise. However, markets are incomplete and Arrow securities are only available for states $1, \cdots, n$, where $n<S$.

An agent's demand for stock and the $i$ 'th Arrow security is denoted by $z_{t}^{0}$ and $z_{t}^{i}$ respectively. Introduce vector notation by setting

$$
\begin{aligned}
\tilde{z}_{t} & =\left(z_{t}^{1}, \cdots, z_{t}^{n}\right) \quad \text { and } \quad z_{t}=\left(z_{t}^{0}, \tilde{z}_{t}\right) ; \\
\tilde{p}_{t} & =\left(p_{t}^{1}, \cdots, p_{t}^{n}\right) \quad \text { and } \quad p_{t}=\left(p_{t}^{0}, \tilde{p}_{t}\right) \\
\delta & =\left(\delta_{1}, \cdots, \delta_{n}\right) \quad \text { and } \\
\alpha & =\left(\alpha_{1}, \cdots, \alpha_{n}\right) .
\end{aligned}
$$

Introduce variance and covariances as

$$
\begin{aligned}
\sigma^{2} & =\mathbb{V a r} q_{t+1}, \\
\eta_{i} & =\mathbb{C o v}\left(q_{t+1}, \delta_{i}\right) \quad \text { and } \quad \eta=\left(\eta_{1}, \cdots, \eta_{n}\right), \\
\Sigma & =\mathbb{C o v}(\delta) .
\end{aligned}
$$

Finally, let $a>0$ be the coefficient of risk aversion and introduce the symmetric $(n+1, n+1)$-matrix

$$
V=a \operatorname{Cov}\left(q_{t+1}, \delta\right)=a\left(\begin{array}{cc}
\sigma^{2} & \eta^{T} \\
\eta & \Sigma
\end{array}\right) .
$$

Note that $V$ is just the covariance matrix of the uncertain payments of the stock and the Arrow securities multiplied by the coefficient of risk aversion $a$. Note also that $V$ is singular if and only if the stochastic variable $q_{t+1}$ is a linear combination of the available Arrow securities. For instance, this will be the case when the market is complete, i.e. when there is a full set of Arrow securities $(n=S)$. 
The inner product of two vectors $v$ and $w$ is denoted by $\langle v, w\rangle$. With this notation, if $W_{t}$ is the current wealth of an agent, his next period's wealth in state of the world $s$ is

$$
W_{t+1}^{s}=R\left(W_{t}-p_{t}^{0} z_{t}^{0}-\left\langle\tilde{p}_{t}, \tilde{z}_{t}\right\rangle\right)+q_{t+1}^{s} z_{t}^{0}+z_{t}^{s} .
$$

In state $s$ the excess profit $\pi_{t+1}^{s}$ due to trading the risky assets equals

$$
\pi_{t+1}^{s}=W_{t+1}^{s}-R W_{t}=\left\langle\left(\begin{array}{l}
-R p_{t}^{0}+q_{t+1}^{s} \\
-R \tilde{p}_{t}+\delta^{s}
\end{array}\right), z_{t}\right\rangle .
$$

Utility is assumed to be of mean-variance type:

$$
U_{t}=\mathbb{E}_{t} \pi_{t+1}-\frac{a}{2} \operatorname{Var}_{t} \pi_{t+1}=\left\langle\left(\begin{array}{l}
-R p_{t}^{0}+\mathbb{E}_{t} q_{t+1} \\
-R \tilde{p}_{t}+\mathbb{E}_{t} \delta
\end{array}\right), z_{t}\right\rangle-\frac{1}{2}\left\langle z_{t}, V z_{t}\right\rangle
$$

Note that this expression is of the form $k(z)=\langle b, z\rangle-\frac{1}{2}\langle z, V z\rangle$, and that $V$ is a positive symmetric matrix. From the identity

$$
\langle b, z\rangle-\frac{1}{2}\langle z, V z\rangle=\frac{1}{2}\left\langle b, V^{-1} b\right\rangle-\frac{1}{2}\left\langle z-V^{-1} b, V\left(z-V^{-1} b\right)\right\rangle,
$$

it follows that $k(z)$ is maximized for $z_{*}=V^{-1} b$, and that $k\left(z_{*}\right)=\frac{1}{2}\left\langle b, V^{-1} b\right\rangle$. Applied to $U_{t}$, this yields the optimal demands

$$
z_{t}=V^{-1}\left(\begin{array}{l}
-R p_{t}^{0}+\mathbb{E}_{t} q_{t+1} \\
-R \tilde{p}_{t}+\mathbb{E}_{t} \delta
\end{array}\right)
$$

2.1 Homogeneous expectations. To obtain a benchmark, consider the case of homogeneous rational expectations. Arrow securities are endogenous to the system and therefore their total supply is zero. The total supply of the stock is $\zeta^{0}$.

Denote expected dividends by $\bar{y}=\sum y^{s} \alpha_{s}$. If the market clears, that is, if total supply equals total demand for the stock and for all Arrow securities, then we obtain using equations (1) and (3)

$$
\begin{aligned}
-R p_{t}^{0}+\mathbb{E}_{t} p_{t+1}^{0}+\bar{y} & =\zeta^{0} a \sigma^{2}, \\
-R \tilde{p}_{t}+\alpha & =\zeta^{0} a \eta, \quad \text { for } i=1, \cdots, n .
\end{aligned}
$$

Imposing the transversality condition that prices remain bounded, these equations are solved by constant fundamental prices $p_{t}=p_{*}$, given as

$$
p_{*}^{0}=\frac{\bar{y}-\zeta^{0} a \sigma^{2}}{R-1}, \quad \tilde{p}_{*}=\frac{1}{R}\left(\alpha-\zeta^{0} a \eta\right) .
$$

The terms involving $\zeta^{0}$ can be interpreted as the risk premium required by the investors to hold the risky assets. These vanish if $\zeta^{0}$ vanishes. 
The elements of the matrix $V$ in (1) can be computed. Recalling $q_{*}^{s}=p_{*}^{0}+y^{s}$, we obtain the variance and the covariances

$$
\begin{aligned}
\sigma^{2} & =\sum\left(y^{s}-\bar{y}\right)^{2} \alpha_{s}, \\
\eta_{i} & =\left(\alpha_{i}\left(y^{i}-\bar{y}\right)-\alpha_{i} \sum_{j} \alpha_{j}\left(y^{j}-\bar{y}\right)\right)=\alpha_{i}\left(y^{i}-\bar{y}\right), \\
\Sigma_{i j} & = \begin{cases}\alpha_{i}\left(1-\alpha_{i}\right) & \text { if } i=j, \\
-\alpha_{i} \alpha_{j} & \text { if } i \neq j .\end{cases}
\end{aligned}
$$

2.2 Heterogeneous expectations. Consider now the case that agents are heterogeneous in their expectations or beliefs about future prices of the stock, but homogeneous with respect to everything else; in particular, we assume that every agent agrees about $V$ having the fundamental value of the homogeneous case.

There are $H$ agent types, indexed by $h$. Equation (3) holds for each agent type, and becomes

$$
z_{h t}=V^{-1}\left(\begin{array}{l}
-R p_{t}^{0}+\mathbb{E}_{h t} q_{t+1} \\
-R \tilde{p}_{t}+\mathbb{E}_{t} \delta
\end{array}\right)=V^{-1} B_{h t}
$$

or equivalently

$$
V z_{h t}=\left(\begin{array}{l}
-R p_{t}^{0}+\mathbb{E}_{h t} q_{t+1} \\
-R \tilde{p}_{t}+\mathbb{E}_{t} \delta
\end{array}\right)=B_{h t}
$$

Here $B_{h t}$ may be interpreted as the belief vector of type $h$ about the excess return of the stock and the Arrow securities. Note that since the probabilities of states of the world are assumed to be common knowledge, the expectation $\mathbb{E}_{t} \delta$ is the same for all types. Also note that agents differ in their assessment of $\mathbb{E}_{h t} q_{t+1}$, but that they agree on $V$. This simplifying assumption is made for analytical tractability of the heterogeneous agent case, but it is supported by the observation that there may be more agreement about the variance than about the mean. The assumptions have parallels in Brock and Hommes (1998); they imply that all agents have the same risk perception as rational fundamentalists.

Recall that $q_{t+1}=p_{t+1}^{0}+y$. We already assumed homogeneous and correct expectations about dividends, but agents have heterogeneous expectations about next period's price of the stock. It will be convenient to work with price deviations from the fundamental benchmark prices given by (4): $x_{t}^{0}=p_{t}^{0}-p_{*}^{0}, x_{t}^{i}=p_{t}^{i}-p_{*}^{i}$. We assume price expectations to be of the form

$$
\mathbb{E}_{h t} p_{t+1}^{0}=p_{*}^{0}+f_{h t}=p_{*}^{0}+f_{h}\left(x_{t-1}, \cdots, x_{t-L}\right) .
$$

Here $f_{h}$ represents a function of past deviations from the fundamental (e.g. a technical trading rule) according to which type $h$ beliefs that prices will deviate from their fundamental benchmark. 
2.2.1 Market clearing. Let the fraction of agents following belief $h$ at time $t$ be denoted as $n_{h t}$. As before, Arrow securities are endogenous to the system, and their total supply is zero. Market clearing for the stock and the Arrow securities requires:

$$
\sum_{h} n_{h t} z_{h t}^{0}=\zeta^{0}, \quad \sum_{h} n_{h t} \tilde{z}_{h t}=0 .
$$

In deviations from the fundamental, equation (6) reads as

$$
V z_{h t}=V\left(\begin{array}{l}
\zeta^{0} \\
0
\end{array}\right)+\left(\begin{array}{l}
-R x_{t}^{0}+f_{h t} \\
-R \tilde{x}_{t}
\end{array}\right)
$$

or equivalently

$$
z_{h t}=\left(\begin{array}{l}
\zeta^{0} \\
0
\end{array}\right)+V^{-1}\left(\begin{array}{l}
-R x_{t}^{0}+f_{h t} \\
-R \tilde{x}_{t}
\end{array}\right) .
$$

Adding these equations, weighted by fractions, and market clearing yields

$$
R x_{t}^{0}=\sum_{h} n_{h t} f_{h t}, \quad \tilde{x}_{t}=0
$$

A number of important observations can now be made. First, according to (9), the price deviations of the Arrow securities are zero, $\tilde{x}_{t}=0$, implying that the Arrow securities are correctly priced. This is due to the fact that Arrow securities are short lived, only one period, and all agent types $h$ have correct beliefs about dividends and their probability distribution. Secondly, when all types are fundamentalists, that is if $f_{h t}=0$ for all $h$, it follows from (9) that also the price deviations of the stock are zero, $x_{t}^{0}=0$. Consequently, by (8), the demand for each Arrow security equals zero; in this case Arrow securities are redundant. Note that this is actually always true when all types $h$ share the same belief, that is, if beliefs are in fact homogeneous, since the total supply of Arrow securities is 0 . In the case of heterogeneous beliefs the demand for Arrow securities will be non-zero, as different types attempt to hedge their risk. Finally, under heterogeneous beliefs about future prices the market price of the risky asset will in general deviate from its fundamental benchmark. In fact, the expression $R x_{t}^{0}=\sum_{h} n_{h t} f_{h t}$ in (9) is the same as in the asset pricing model without Arrow securities in Brock and Hommes (1998). However, as we will see below, the existence of Arrow securities will affect the magnitude of the fractions $n_{h t}$ through the evolutionary updating mechanism.

2.2.2 Fitness. In order to close the model, the evolution of the market shares $n_{h t}$ has to be specified. We assume that their market share is related to their fitness, measured by some $u_{h t-1}$; the subscript $t-1$ indicates that the fitness measure depends only on past prices that are known. The fraction of agents using strategy type $h$ will thus be driven by "experience" or "regret" through reinforcement learning. Given the fitness 
measure, the fraction of agents using strategy type $h$ is determined by a multinomial logit model:

$$
n_{h t}=\frac{\mathrm{e}^{\beta u_{h t-1}}}{Z_{t}}, \quad Z_{t}=\sum_{h} \mathrm{e}^{\beta u_{h t-1}} .
$$

where $Z_{t}$ is a normalization factor for the fractions $n_{h t}$ to add up to 1 . These fractions are derived from a random utility model. Manski and McFadden (1981) and Anderson, de Palma and Thisse (1993) give an extensive overview and discussion of discrete choice models, in particular the multinomial logit model, and their applications in economics. Brock and Hommes (1997) have applied this framework to selection of expectations rules.

The crucial feature of (10) is that the higher the fitness of trading strategy $h$, the more agents will select strategy $h$. The intensity of choice parameter $\beta>0$ in (10) measures how sensitive agents are to selecting the optimal prediction strategy. This intensity of choice $\beta$ is inversely related to the variance of the noise in the observation of random utility. The extreme case $\beta=0$ corresponds to noise with infinite variance, so that differences in fitness cannot be observed and all fractions (10) will be equal to $1 / H$. The other extreme case $\beta=+\infty$ corresponds to the case without noise, so that the deterministic part of the fitness is observed perfectly and in each period, all agents choose the optimal forecast. An increase in the intensity of choice $\beta$ represents an increase in the degree of rationality with respect to evolutionary selection of strategies.

We look at two fitness measures for strategies: average profits and average riskadjusted profits.

Average profits. The first fitness measure to be considered is the average profit due to trading risky assets, obtained by averaging actual profits $\pi_{h t}^{s}$ over the states of the world:

$$
\begin{aligned}
u_{h t}= & \left\langle\left(\begin{array}{l}
-R p_{t-1}^{0}+p_{t}^{0}+\bar{y} \\
-R \tilde{p}_{t-1}+\alpha
\end{array}\right), z_{h t-1}\right\rangle \\
= & \left\langle V\left(\begin{array}{c}
\zeta^{0} \\
0
\end{array}\right)+\left(\begin{array}{c}
-R x_{t-1}^{0}+x_{t}^{0} \\
0
\end{array}\right),\right. \\
& \left.\left(\begin{array}{c}
\zeta^{0} \\
0
\end{array}\right)+V^{-1}\left(\begin{array}{c}
-R x_{t-1}^{0}+f_{h t-1} \\
0
\end{array}\right)\right\rangle .
\end{aligned}
$$

Since the $n_{h t}$ depend only on fitness differences, not on the absolute level of fitness, the fitness measure $u_{h t}$ is split in a term $u_{0 t}$, which is the same for all types, and a type-dependent contribution. It reads as:

$$
u_{h t}=u_{0 t}+\left\langle\left(\begin{array}{c}
-R x_{t-1}^{0}+x_{t}^{0} \\
0
\end{array}\right)+V\left(\begin{array}{l}
\zeta^{0} \\
0
\end{array}\right), V^{-1}\left(\begin{array}{l}
f_{h t-1} \\
0
\end{array}\right)\right\rangle .
$$


Since $u_{0 t}$ is independent of $h$ and the discrete choice fractions (10) are independent of the fitness level we can drop the term $u_{0 t}$. The matrices $V$ and $V^{-1}$ are symmetric. After shifting $V^{-1}$ to the other factor of the inner product and dropping $u_{0 t}$, the product can be evaluated, yielding

$$
u_{h t}=\left(V^{-1}\right)_{00}\left(x_{t}^{0}-R x_{t-1}^{0}\right) f_{h t-1}+\zeta^{0} f_{h t-1},
$$

where the subindex 00 refers to the element in the first column and first row in the matrix (the row and column corresponding to the stock).

Average risk-adjusted profits. The second fitness measure is average risk-adjusted profit, that is, average profits corrected for the risk taken when buying risky assets. Average risk-adjusted profits is given by

$$
u_{h t}=\left\langle\left(\begin{array}{l}
-R p_{t-1}^{0}+p_{t}^{0}+\bar{y} \\
-R \tilde{p}_{t-1}+\alpha
\end{array}\right), z_{h t-1}\right\rangle-\frac{1}{2}\left\langle z_{h t-1}, V z_{h t-1}\right\rangle .
$$

Notice that this fitness measure for strategy selection is consistent with mean-variance maximization in (2). Using (5) and the realized excess return vector

$$
B_{t-1}=\left(\begin{array}{l}
-R p_{t-1}^{0}+p_{t}^{0}+\bar{y} \\
-R \tilde{p}_{t-1}+\alpha
\end{array}\right),
$$

we can rewrite risk-adjusted realized profits as

$$
\begin{aligned}
u_{h t} & =\left\langle B_{t-1}, z_{h, t-1}\right\rangle-\frac{1}{2}\left\langle z_{h, t-1}, V z_{h, t-1}\right\rangle \\
& =\left\langle B_{t-1}, V^{-1} B_{h, t-1}\right\rangle-\frac{1}{2}\left\langle V^{-1} B_{h, t-1}, V V^{-1} B_{h, t-1}\right\rangle \\
& =\left\langle B_{t-1}, V^{-1} B_{h, t-1}\right\rangle-\frac{1}{2}\left\langle B_{h, t-1}, V^{-1} B_{h, t-1}\right\rangle .
\end{aligned}
$$

In the special case where type $h$ has rational expectations or perfect foresight, i.e. $B_{h, t-1}=B_{t-1}$, this expression simplifies to $u_{t}^{R}=\frac{1}{2}\left\langle B_{t-1}, V^{-1} B_{t-1}\right\rangle$. Now look at the difference between risk-adjusted profits of type $h$ and fully rational agents, i.e.

$$
\begin{aligned}
u_{h t}-u_{t}^{R} & =\left\langle B_{t-1}, V^{-1} B_{h, t-1}\right\rangle-\frac{1}{2}\left\langle B_{h, t-1}, V^{-1} B_{h, t-1}\right\rangle-\frac{1}{2}\left\langle B_{t-1}, V^{-1} B_{t-1}\right\rangle \\
& \left.=-\frac{1}{2}\left\langle B_{t-1}-B_{h, t-1}\right), V^{-1}\left(B_{t-1}-B_{h, t-1}\right)\right\rangle \\
& =-\frac{1}{2}\left\langle\left(\begin{array}{l}
x_{t}-f_{h, t-1} \\
0
\end{array}\right), V^{-1}\left(\begin{array}{l}
x_{t}-f_{h, t-1} \\
0
\end{array}\right)\right\rangle \\
& =-\frac{1}{2}\left(V^{-1}\right)_{00}\left(x_{t}-f_{h, t-1}\right)^{2} .
\end{aligned}
$$

Since $u_{t}^{R}$ is independent of $h$ and the fractions are independent of the fitness level we conclude that risk-adjusted profits are equivalent, up to a constant factor, to (minus) squared prediction errors. In the case when there are no Arrow securities we have $\left(V^{-1}\right)_{00}=1 /\left(a \sigma^{2}\right)$ and the risk adjustment fitness measure coincides with Brock and Hommes (1998). 
2.3 Adding Arrow securities. This subsection addresses the main theme of this paper in the asset pricing setting with heterogeneous beliefs: what happens to the dynamics under reinforcement learning when adding Arrow securities?

2.3.1 General mechanism To make the dependence on the number of Arrow securities explicit, we write $V_{n}$ for the $(n+1, n+1)$-matrix (1) in the case with $n$ Arrow securities. When we add an extra Arrow security to the system, the dynamical behavior only changes through the term $\left(V_{n}^{-1}\right)_{00}$ in the fitness measure. Adding the $(n+1)$-th Arrow security the corresponding symmetric $(n+2, n+2)$-matrix takes the form

$$
V_{n+1}=\left(\begin{array}{cc}
V_{n} & r \\
r^{T} & s
\end{array}\right)
$$

where

$$
r=\left(a \operatorname{Cov}\left(q_{t+1}, \delta_{n+1}\right), a \mathbb{C o v}\left(\delta_{1}, \delta_{n+1}\right), \cdots, a \mathbb{C o v}\left(\delta_{n}, \delta_{n+1}\right)\right),
$$

and

$$
s=a \operatorname{Var}\left(\delta_{n+1}\right) .
$$

To obtain information about $\left(V_{n+1}^{-1}\right)_{00}$, the following matrix lemma is useful. The proof of the first part of this lemma can be established by a variation on the use of the formula for the inverse of a partitioned matrix which uses the notion of Schur complement of a submatrix of a matrix (Skogestad and Postlethwaite (1996, p. 499). The second part can be established using Schur's formula for the determinant of a partitioned matrix (Skogestad and Postlethwaite (1996, p. 500)). In appendix A we give a self contained proof.

LEMMA 1. Let $Q_{n}$ be a symmetric $(n, n)$-matrix and $Q_{n+1}$ a symmetric $(n+1, n+1)$ matrix of the form $\left(\begin{array}{cc}Q_{n} & r \\ r^{T} & s\end{array}\right)$, where $r$ is an n-vector and $s$ a scalar, and let $\tilde{w}=$ $\left(w, w_{0}\right)$, with $w$ an $n$-vector and $w_{0}$ a scalar. Then

$$
\left\langle\tilde{w}, Q_{n+1}^{-1} \tilde{w}\right\rangle=\left\langle w, Q_{n}^{-1} w\right\rangle+\frac{\left(w_{0}-\left\langle r, Q_{n}^{-1} w\right\rangle\right)^{2}}{s-\left\langle r, Q_{n}^{-1} r\right\rangle} .
$$

Moreover,

$$
\operatorname{det} Q_{n+1}=\operatorname{det} Q_{n}\left(s-\left\langle r, Q_{n}^{-1} r\right\rangle\right) .
$$

Note that if $w=(1,0, \cdots, 0)$, then $\left(V_{n}^{-1}\right)_{00}=\left\langle w, V_{n}^{-1} w\right\rangle$. Since both $V_{n}$ and $V_{n+1}$ are symmetric positive matrices, we have $\operatorname{det} V_{n}$, $\operatorname{det} V_{n+1}>0$. Apply the lemma to see that

$$
s-\left\langle r, Q_{n}^{-1} r\right\rangle=\frac{\operatorname{det} Q_{n+1}}{\operatorname{det} Q_{n}}>0
$$


and consequently that

$$
\left(V_{n+1}^{-1}\right)_{00}=\left\langle\tilde{w}, V_{n+1}^{-1} \tilde{w}\right\rangle \geq\left\langle w, V_{n}^{-1} w\right\rangle=\left(V_{n}^{-1}\right)_{00} .
$$

In fact, (15) is a strict inequality except for hairline cases. This can be seen by noting that since $w_{0}=0$ and $w=e_{1}$ is the first unit vector in our application of the lemma, the inequality will be strict if and only if $\left\langle r, V_{n}^{-1}\left(e_{1}\right)\right\rangle \neq 0$, which will be the case for typical choices of the dividends $y^{s}$ and the probabilities $\alpha_{s}$, except for hairline cases. It may be more intuitive to work with

$$
\sigma_{n}^{2}=\frac{1}{a\left(V_{n}^{-1}\right)_{00}}, \quad 0 \leq n \leq S-1,
$$

which may be viewed as a measure of risk when there are $n$ Arrow securities. The (strict) inequalities (15) are equivalent to

$$
\sigma_{0}^{2}>\sigma_{1}^{2}>\cdots>\sigma_{S-2}^{2}>\sigma_{S-1}^{2}=0
$$

implying that the risk measure decreases when more Arrow securities are added to the market, because more risk can be hedged.

We are now ready to formulate the main result within the mean-variance heterogeneous agent asset pricing framework. A typical feature of reinforcement or evolutionary learning systems as in Brock and Hommes $(1997,1998)$ is a bifurcation route to instability and complicated dynamics when the intensity of choice $\beta$ to switch strategies increases. We claim that adding Arrow securities leads to earlier primary bifurcations:

THEOREM 1. Consider the asset price dynamics with reinforcement learning in (910). Let the fitness measure be given either by (i) average profits in (11) with zero supply of outside shares, i.e. $\zeta^{0}=0$, or (ii) average risk-adjusted profits in (13). If $\beta_{0}^{*}$ is the primary bifurcation value in the case without Arrow securities, that is, $\beta_{0}^{*}$ is the critical value for which the steady state becomes unstable if there are no Arrow securities, then for almost all dividends $y^{s}$ and probabilities $\alpha_{s}$ the primary bifurcation value $\beta_{n}^{*}$ for the system with $n$ Arrow securities and incomplete markets (i.e. $n<S$ ) satisfies

$$
\beta_{n+1}^{*}<\beta_{n}^{*}<\beta_{0}^{*}, \quad 1 \leq n<S-2 .
$$

\section{Proof}

The proof follows immediately from the inequality (15) or equivalently, the inequalities (17). As discussed above, this inequality is strict if and only if $\left\langle r, V_{n}^{-1}\left(e_{1}\right)\right\rangle \neq 0$, where

$$
r=\left(a \operatorname{Cov}\left(q_{t+1}, \delta_{n+1}\right), a \mathbb{C o v}\left(\delta_{1}, \delta_{n+1}\right), \cdots, a \operatorname{Cov}\left(\delta_{n}, \delta_{n+1}\right)\right),
$$


which will be the case for Lebesgue almost all choices of $y^{s}$ and $\alpha_{s}$. The fitness given by average risk-adjusted profits (13) is proportional to $\left(V_{n}^{-1}\right)_{00}$ or, equivalently, inversely proportional to $\sigma_{n}^{2}$. The same holds for the fitness given by average profits (11), when $\zeta^{0}=0$. Let $\beta_{0}^{*}$ be the first bifurcation value when there are no Arrow securities. Then the system with $n$ Arrow securities will undergo its first bifurcation if

$$
\frac{\beta}{\sigma_{n}^{2}}=\frac{\beta_{0}^{*}}{\sigma_{0}^{2}}, \quad \text { that is, if } \beta=\beta_{0}^{*} \frac{\sigma_{n}^{2}}{\sigma_{0}^{2}} \stackrel{\text { def }}{=} \beta_{n}^{*}
$$

From (17) we infer that

$$
\beta_{0}^{*}>\beta_{1}^{*}>\cdots>\beta_{S-2}^{*}
$$

Consequently, with more Arrow securities the primary bifurcation comes earlier, that is $\beta_{n+1}^{*}<\beta_{n}^{*}<\beta_{0}^{*}, 1 \leq n<S-2$.

This theorem implies that, in the presence of more Arrow securities, the primary bifurcation at the onset of instability occurs earlier. In fact, if all other parameters including the intensity of choice are fixed, adding Arrow securities may destabilize the market. Within the mean-variance asset pricing framework this result holds in general for the average risk-adjusted profit fitness measure. For the average profit fitness measure the result can not be shown in full generality because of the extra, type dependent, term $\zeta^{0} f_{h, t-1}$ in the fitness measure (11). When outside supply of shares is zero, i.e. $\zeta^{0}=0$, this term drops from the fitness and the result holds. If $\zeta^{0}>0$, the result may or may not hold depending on the distribution of belief types.

There is a simple economic intuition behind the theorem. When there are more Arrow securities, agents will take bigger positions in the risky asset because they can hedge out more risk. Moreover, trading strategies that turn out to be on the right side of the market will earn higher rewards and, under reinforcement learning, will attract more followers. This may be seen from substituting the Arrow security equilibrium prices $\tilde{x}_{t} \equiv 0$ from (9) into the demand vector (8) to obtain

$$
z_{h t}=\left(\begin{array}{c}
\zeta^{0} \\
0
\end{array}\right)+V_{n}^{-1}\left(\begin{array}{c}
-R x_{t}^{0}+f_{h t} \\
0
\end{array}\right) .
$$

The demand of type $h$ for the stock is then given by

$$
z_{h t}^{0}=\left(V_{n}^{-1}\right)_{00}\left(f_{h t}-R x_{t}^{0}\right)=\frac{\left(f_{h t}-R x_{t}^{0}\right)}{a \sigma_{n}^{2}} .
$$

When the number of Arrow securities increases, the risk measure $\sigma_{n}^{2}$ decreases. Hence, it is clear from (21) that the introduction of additional Arrow securities forces optimistic (pessimistic) agents, with the same risk aversion coefficient $a$, to hold bigger (smaller) positions in the stock. For example, optimistic traders who predict next period's asset price deviation $f_{h t}$ from the fundamental price to grow faster than $R$ times 
the current positive deviation, that is, for whom $f_{h t}-R x_{t}^{0}>0$, will take larger positions when there are more Arrow securities. Agents hold bigger positions because in the presence of more Arrow securities they hedge out more risk. Moreover, strategies that more accurately forecasted the price movement will attract more followers according to the risk-adjusted fitness measure (13) and inequality (15). Similarly, strategies that more accurately predicted the excess return $x_{t}^{0}-R x_{t-1}^{0}$ will earn higher average profits when there are more Arrow securities according to (11) and thus also attract more followers. Stated differently, strategies that turned out to be on the "right" side of the market will be rewarded and attract more followers.

2.4 Examples. In this subsection we present simple examples of bifurcation routes to instability in the asset pricing model with heterogeneous beliefs and reinforcement learning. In the first example the fitness measure is average realized profit, while in the second example fitness equals average risk adjusted profits. In both examples, adding Arrow securities destabilizes the system earlier and leads by a bifurcation route to more complicated dynamical behavior.

Example 1. In the first example we take average profits (11) as the fitness measure. Consider an example with three different purely biased forecasting rules. More precisely, let $b>0$ be a constant, and let the forecast rules be given, in terms of deviations from the fundamental benchmark, by:

$$
\begin{aligned}
& f_{1 t}=0, \\
& f_{2 t}=b, \\
& f_{3 t}=-b,
\end{aligned}
$$

Agents of type 1 are fundamentalists, who believe that prices will always be at their fundamental value, or, equivalently, who expect price deviations from the fundamental to be zero. Type 2 are optimists, who expect that the price of the good will always be a constant amount $b$ above the fundamental price, whereas type 3 are pessimists, who always expect prices to be a constant amount $b$ below the fundamental price. This example is symmetric in the sense that the optimistic and the pessimistic strategy are exactly balanced around the fundamental price. A consequence of this symmetry is that the steady state price of the system coincides exactly with the fundamental price.

Brock and Hommes (1998, pp. 1258-1261) have studied this example in detail; they have showed that a Hopf bifurcation occurs when the intensity of choice $\beta$ increases. For $b=0.2$ and no Arrow securities, the Hopf bifurcation occurs for $\beta=\beta_{0}^{*} \approx 37.5$. Figure 1 plots the coefficient $\beta\left(V_{n}^{-1}\right)_{00}=\beta /\left(a \sigma_{n}^{2}\right)$, where $\beta=3$, as a function of the number $n$ of Arrow securities. According to lemma 1 this coefficient is (strictly) increasing, and the figure illustrates that it blows up when the market approaches completeness, that is, as the number of Arrow securities approaches the number of states of the world, $S=40$. Without Arrow securities the system is stable, since 


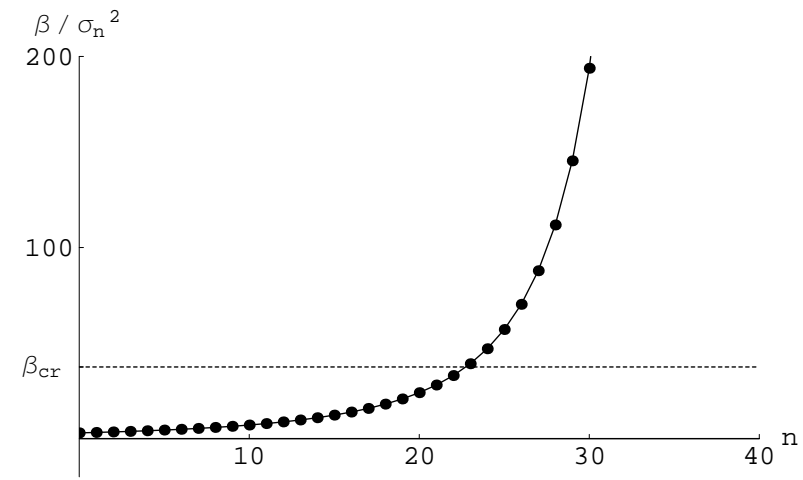

(a) Uniform distribution

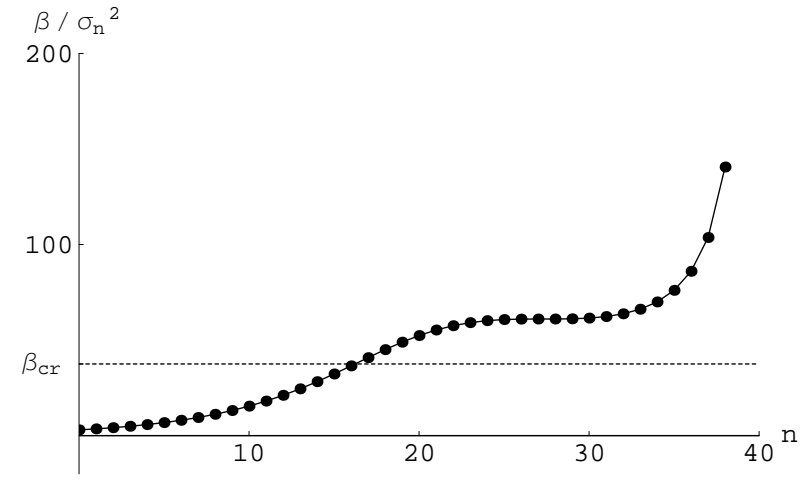

(b) Geometric distribution

Figure 1: Plot of the coefficient $\beta\left(V_{n}^{-1}\right)_{00}=\beta /\left(a \sigma_{n}^{2}\right)$ as a function of the number $n$ of Arrow securities, for two different probability distributions. There are $S=40$ states of the world, $(a)$ in the left plot all with equal probabilities $\alpha_{i}=1 / S,(b)$ in the right plot with probabilities $\alpha_{i}=p^{i}(1-p), i=1, \cdots, S-1$, and $\alpha_{S}=1-\sum_{1}^{S-1} \alpha_{i}$, with $p=6 / 7$. Dividends in state of the world $i$ are given by $y_{i}=i-1$. Other parameters are $\beta=3, b=0.2$ and $\zeta^{0}=0$. Without Arrow securities $\left(V^{-1}\right)_{00}=1 /\left(a \sigma^{2}\right)=1$, but $\left(V_{n}^{-1}\right)_{00}=1 /\left(a \sigma_{n}^{2}\right)$ increases as the number of Arrow securities increases and explodes when $n$ approaches the number of states $S=40$. When the number of Arrow securities increases from 22 to 23 for the uniform and from 16 to 17 for the geometric distribution, the critical Hopf bifurcation value (indicated by the dotted line) is crossed, and the system is destabilized. 
$\beta=3<\beta_{0}^{*}=37.5$. As the number $n$ of Arrow securities increases, the product $\beta\left(V_{n}^{-1}\right)_{00}=\beta /\left(a \sigma_{n}^{2}\right)$ crosses the critical bifurcation value $\beta_{0}^{*}=37.5$ (indicated by the dotted line), and the system becomes unstable when the number of Arrow securities increases from 22 to 23 .

Example 2 Next consider an example with average risk-adjusted profits as the fitness measure. This example is important, because it will also arise as a quadratic approximation around a steady state in the overlapping generations general equilibrium framework in Section 3.

There are two types of traders, (near-)fundamentalists versus trend extrapolators, with forecasting rules (in deviations from the fundamental benchmark):

$$
\begin{aligned}
& f_{1 t}=\varepsilon \\
& \left.f_{2 t}=x_{t-1}+g\left(x_{t-1}-x_{t-2}\right)\right) .
\end{aligned}
$$

Type 1 agents use information about economic fundamentals and predict that the price of the risky asset will be equal to its fundamental value. However, they make a (small) error, $\varepsilon$, in computing this fundamental value. Type 2 are trend followers who do not use fundamental information, but extrapolate the latest observed price change by an extrapolation factor $g$. As before, agents try to learn the best forecasting strategy, with fitness given by risk-adjusted profits, which according to (13) are proportional to minus quadratic prediction errors; without loss of generality, we can put the constant of proportionality equal to 1 , so that fitnesses of the two strategies are given by:

$$
u_{1 t}=-\left(x_{t-1}-\varepsilon\right)^{2}, \quad u_{2 t}=-\left(x_{t-1}-(1+g) x_{t-3}+x_{t-4}\right)^{2} .
$$

Market fractions of the two types are given as

$$
n_{1 t}=\frac{\mathrm{e}^{\beta u_{1}}}{\mathrm{e}^{\beta u_{1}}+\mathrm{e}^{\beta u_{2}}}=\frac{1}{1+\mathrm{e}^{-\beta\left(u_{1}-u_{2}\right)}}, \quad n_{2 t}=1-n_{1 t} .
$$

Market equilibrium (9) gives the dynamics of the system

$$
R x_{t}=n_{1 t} \varepsilon+\left(1-n_{1 t}\right)\left(x_{t-1}+g\left(x_{t-1}-x_{t-2}\right)\right) .
$$

To simplify the notation in the discussion below, it is convenient to introduce scaled coordinates and scaled parameters by setting $x_{t}=\varepsilon \tilde{x}_{t}$ and $\beta=\varepsilon^{-2} \tilde{\beta}$, so that

$$
\begin{aligned}
& \beta u_{1}=\tilde{\beta} \tilde{u}_{1}=-\tilde{\beta}\left(\tilde{x}_{t-1}-1\right)^{2}, \\
& \beta u_{2}=\tilde{\beta} \tilde{u}_{2}=-\tilde{\beta}\left(\tilde{x}_{t-1}-\tilde{x}_{t-3}-g\left(\tilde{x}_{t-3}-\tilde{x}_{t-4}\right)\right)^{2}, \\
& R \tilde{x}_{t}=\frac{1}{1+\mathrm{e}^{\tilde{\beta}\left(\tilde{u}_{1}-\tilde{u}_{2}\right)}}+\left(1-\frac{1}{1+\mathrm{e}^{\tilde{\beta}\left(\tilde{u}_{1}-\tilde{u}_{2}\right)}}\right)\left(\tilde{x}_{t-1}+g\left(\tilde{x}_{t-1}-\tilde{x}_{t-2}\right)\right) .
\end{aligned}
$$

In what follows we work in scaled variables, but to ease notation we drop all tildes. 
Steady states. The system (28) has a steady state equilibrium $x_{*} \in \mathbb{R}$ if $x_{*}$ satisfies the equation

$$
R x_{*}=\frac{1}{1+\mathrm{e}^{\beta\left(x_{*}-1\right)^{2}}}+\left(1-\frac{1}{1+\mathrm{e}^{\beta\left(x_{*}-1\right)^{2}}}\right) x_{*} .
$$

To show that our example actually has a steady state, we consider the function

$$
F_{\beta, R}(x)=n_{\beta}(x)+\left(1-n_{\beta}(x)\right) x-R x,
$$

where

$$
n_{\beta}(x)=\frac{1}{1+\mathrm{e}^{\beta(x-1)^{2}}} .
$$

If $F_{\beta, R}\left(x_{*}\right)=0$, then the price evolution $x_{t}=x_{*}$ for all $t$ is a steady state equilibrium of the dynamics in (28). We note that

$$
\begin{aligned}
F_{\beta, R}(0) & =n_{\beta}(0)>0, \\
F_{\beta, R}(1 / R) & =n_{\beta}(1 / R)+\left(1-n_{\beta}(1 / R)\right) \frac{1}{R}-1 \\
& =\left(1-n_{\beta}(1 / R)\right)\left(-1+\frac{1}{R}\right)<0 .
\end{aligned}
$$

By the intermediate value theorem, there exists at least one $x_{*} \in(0,1 / R)$ such that $F\left(x_{*}\right)=0$. For either $\beta>0$ sufficiently close to 0 or $\beta$ sufficiently large, it can be shown that this steady state equilibrium is actually unique; for intermediate values of $\beta$, there may be several steady states. In fact, we can parametrize the steady states as a function of $\beta$, by solving equation (29) for $\beta$. This yields

$$
\beta=\beta\left(x_{*}\right)=\frac{1}{\left(1-x_{*}\right)^{2}} \log \frac{1-R x_{*}}{(R-1) x_{*}} .
$$

The graph of this function is illustrated in Figure 2. In appendix B it is shown that, as the intensity of choice $\beta$ increases, the following bifurcation scenario occurs. For $\beta=0$, the steady state $x_{*}=1 /(2 R-1)=1 /(1+2 r) \approx 1$ (recall that $r=R-1$ ). Since we are working in scaled variables, this steady state is close to $\varepsilon$, the predicted steady state of type 1 . As $\beta$ increases, the steady state $x_{*}(\beta)$ moves along the upper part of the curve in Figure 2, and this steady state is stable. For $\beta=\beta_{\mathrm{SN}_{1}} \approx 5.5$ two additional steady states are created in a saddle-node bifurcation, one stable (the lower one) and one unstable (the middle one). Note that these two steady states are closer to the fundamental value $x \equiv 0$. As $\beta$ increases, the steady state closest to the fundamental values loses stability through a Hopf bifurcation at $\beta_{\mathrm{Hopf}} \approx 11.0$. At $\beta_{\mathrm{SN}_{2}} \approx 13.6 \mathrm{a}$ second saddle-node bifurcation occurs, and the two upper steady states disappear. For $\beta_{\mathrm{Hopf}}<\beta<\beta_{\mathrm{SN}_{2}}$ a stable steady states co-exists with an attractor around the fundamental steady state. Figure 3 shows a bifurcation diagram and a Lyapunov exponent 


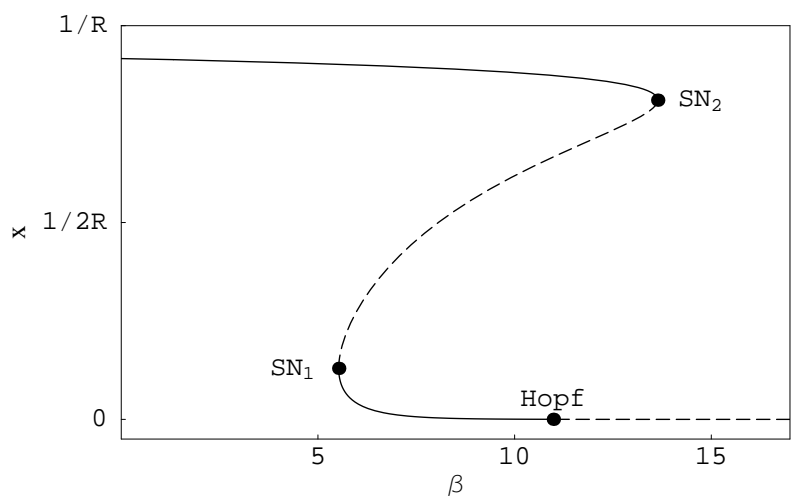

Figure 2: Steady state bifurcation diagram of the system (28) for $R=1.1$ in scaled coordinates. Values of $\beta$ are on the horizontal axis, values of $x$ on the vertical axis. The curve shown is the locus of the steady state equilibria $x_{*}$. Note that there occur two saddle node (SN) bifurcations and one Hopf bifurcation, and that $x_{*} \rightarrow 0$, the true fundamental, as $\beta \rightarrow \infty$. To obtain unscaled coordinates, multiply the values on the horizontal axis by $\varepsilon^{-2}$ and those on the vertical axis by $\varepsilon$.

plot, illustrating the dynamical behavior after the Hopf bifurcation. After the Hopf bifurcation (quasi-)periodic behavior occurs with a Lyapunov exponent close to 0 . For large values of $\beta$ the dynamics becomes chaotic, with positive Lyapunov exponent. Introduction of additional Arrow securities has the same effect as increasing the parameter $\beta$. For example, with $S=40$ states of the world, all with equal probabilities $\alpha_{i}=1 / S$, and dividends $y_{i}=i-1$, as in the previous example, fixing $\beta=1$ yields the following dynamics depending upon the number $n$ of Arrow securities (see Figure 4):

(i) unique stable steady state for $n=0$ and $n=1$;

(ii) co-existence of two stable steady states for $2 \leq n \leq 9$;

(iii) co-existence of stable steady state and (quasi-)periodic attractor for $n=10$ and $n=11$;

(iv) (quasi-)periodic attractor, for $12 \leq n \leq 32$

(v) chaotic behavior, for $33 \leq n<S=40$ 


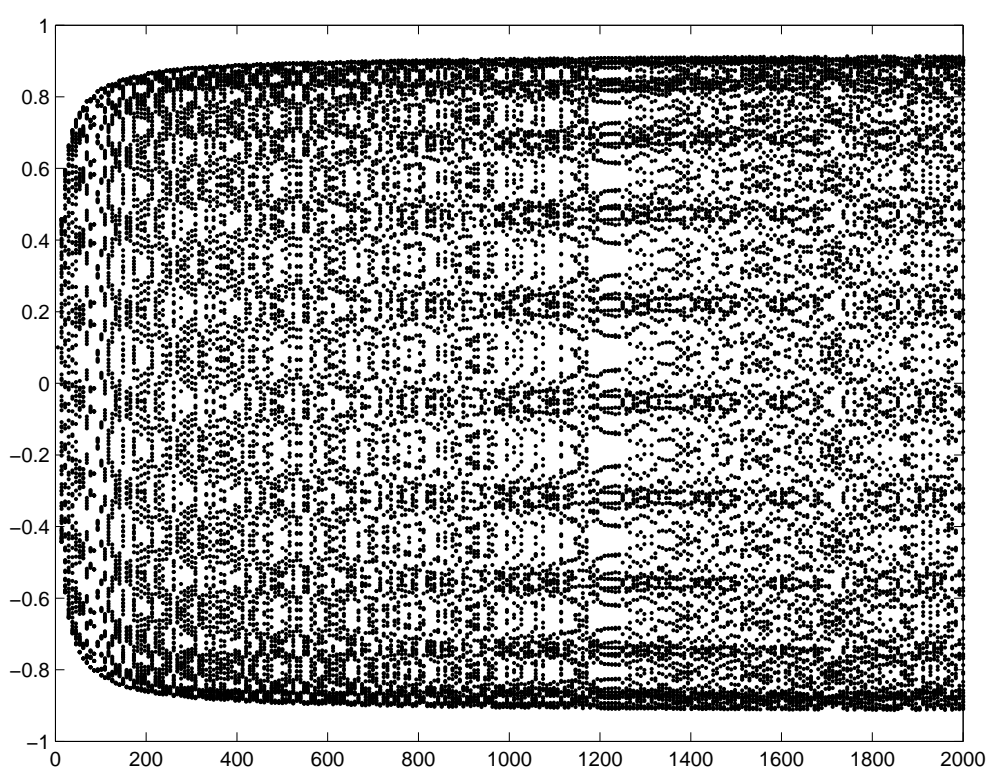

(a) Bifurcation diagram

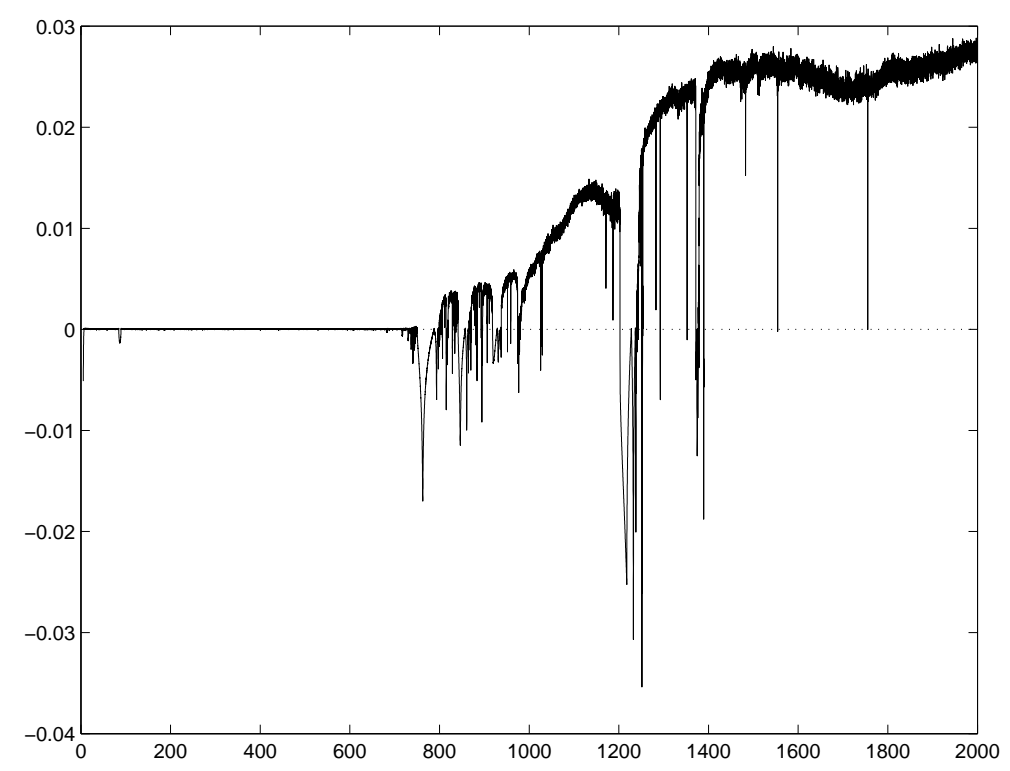

(b) Lyapunov exponents

Figure 3: Bifurcation diagram (a) and Lyapunov exponent plot (b) of system (28) for $R=$ $1.1, g=1.101$ and $\varepsilon=1$ in scaled coordinates. Values of $\beta$ are on the horizontal axis, values of $x$ and the first Lyapunov exponent are on the vertical axis in the upper and lower plot respectively. 


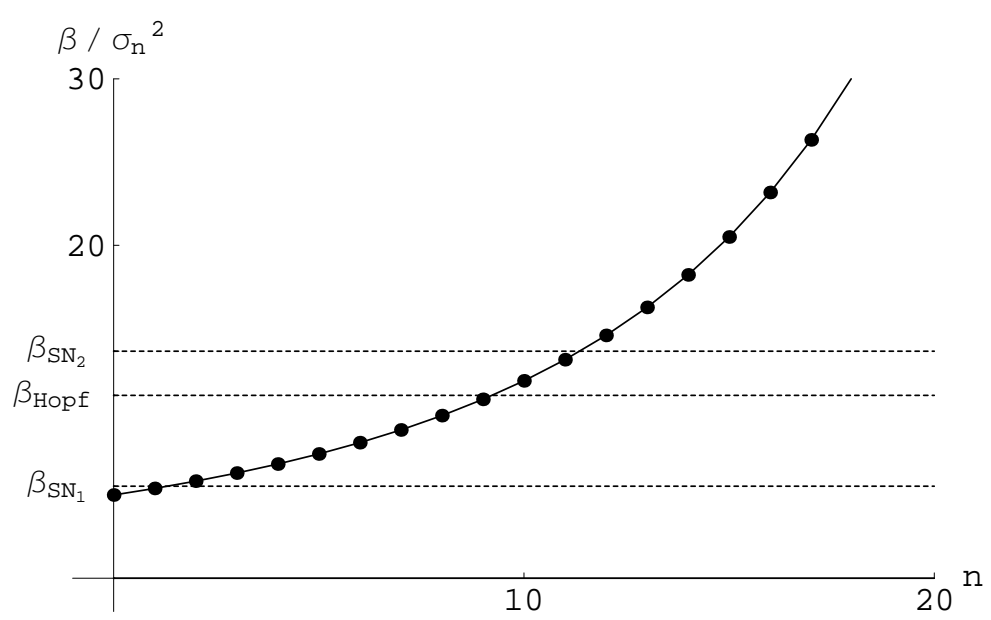

Figure 4: Plot of the coefficient $\beta\left(V_{n}^{-1}\right)_{00}=\beta /\left(a \sigma_{n}^{2}\right)$ as a function of the number $n$ of Arrow securities (scaled variables). There are $S=40$ states of the world, all with equal probabilities $\alpha_{i}=1 / S$, with dividends $y_{i}=i-1$. Other parameters are $\beta=1$ and $R=1.1$. Without Arrow securities $\left(V^{-1}\right)_{00}=1 /\left(a \sigma^{2}\right)=1$, but $\left(V_{n}^{-1}\right)_{00}=1 /\left(a \sigma_{n}^{2}\right)$ increases as the number of Arrow securities increases and explodes when $n$ approaches the number of states $S=40$. The first saddle node bifurcation value is crossed when the number of Arrow securities increases from 1 to 2, the Hopf bifurcation value, where the system is destabilized, is crossed at the increase from 9 to 10, and the second saddle node value is crossed at the increase from 11 to 12.

\section{Two period overlapping generations model}

In this section we study reinforcement learning in a general equilibrium two period overlapping generations (OG) framework. OG models with adaptive learning have e.g. been studied by Grandmont (1985), Bullard (1994), Marcet and Sargent (1989), Schönhofer (1999) and Bullard and Duffy (2001). A novel feature of this section is that we consider heterogeneous beliefs and investigate how Arrow securities affect the stability of the system. An earlier OG model with heterogeneous beliefs (but without Arrow securities) has been studied in Brock and DeFontnouvelle (2000).

3.1 Setup. Consider a world where agents live for two periods. They have endowments $w_{1}$ and $w_{2}$ in their respective 'young' and 'old' periods. Their consumptions $c_{1}$ and $c_{2}$ in these periods give them utility equal to $u_{1}\left(c_{1}\right)+u_{2}\left(c_{2}\right)$. The functions $u_{1}$ and $u_{2}$ are assumed to be continuous on $[0, \infty)$, (infinitely) differentiable on $(0, \infty)$, monotonically increasing and to have a strictly negative second derivative everywhere; in particular, they are assumed to be strictly concave. Moreover, it is assumed that $u_{i}^{\prime}(c) \rightarrow \infty$ as $c \rightarrow 0$ for $i=1,2$.

Young agents can consider to sell part of their endowment to the old agents living at the same time, obtaining either a long-lived risky asset that pays an uncertain dividend $y^{s}$ in the next period, or an Arrow security for state $i$, paying $\delta_{i}^{s}$ in state $s$. As before, 
there are $S$ states of the world and $n$ Arrow securities, $0 \leq n<S$. The price of the consumptions good is normalized to 1 . Young agents demand $z_{t}^{0}$ units of the risky asset at the market price $p_{t}^{0}$, and $z_{t}^{i}$ units of the $i$ 'th Arrow security at price $p_{t}^{i}$, subject to their budget constraints

$$
c_{1 t}+p_{t}^{0} z_{t}^{0}+\left\langle\tilde{p}_{t}, \tilde{z}_{t}\right\rangle=w_{1}, \quad c_{2 t}^{s}=w_{2}+\left(p_{t+1}^{0}+y^{s}\right) z_{t}^{0}+\left\langle\delta^{s}, \tilde{z}_{t}\right\rangle,
$$

where we use the same vector notation $\tilde{z}_{t}=\left(z_{t}^{1}, \cdots, z_{t}^{n}\right)$ and $\tilde{p}_{t}=\left(p_{t}^{1}, \cdots, p_{t}^{n}\right)$ as before. The demand $z_{t}^{0}$ for the risky asset and $\tilde{z}_{t}$ for the Arrow security maximizes their expected utility

$$
\begin{aligned}
U_{t} & =u_{1}\left(c_{1 t}\right)+\mathbb{E}_{t} u_{2}\left(c_{2 t}\right) \\
& =u_{1}\left(w_{1}-p_{t}^{0} z_{t}^{0}-\left\langle\tilde{p}_{t}, \tilde{z}_{t}\right\rangle\right)+\mathbb{E}_{t} u_{2}\left(w_{2}+\left(p_{t+1}^{0}+y^{s}\right) z_{t}^{0}+\left\langle\delta^{s}, \tilde{z}_{t}\right\rangle\right) .
\end{aligned}
$$

Assuming $u_{1}$ and $u_{2}$ to be strictly increasing smooth concave functions, utility is maximized whenever the derivatives of $U_{t}$ with respect to the demands vanish:

$$
\frac{\partial U_{t}}{\partial z_{t}}=\left(\frac{\partial U_{t}}{\partial z_{t}^{0}}, \frac{\partial U_{t}}{\partial \tilde{z}_{t}}\right)=(0,0)
$$

where

$$
\begin{aligned}
\frac{\partial U_{t}}{\partial z_{t}^{0}}=-u_{1}^{\prime}\left(w_{1}\right. & \left.-p_{t}^{0} z_{t}^{0}-\left\langle\tilde{p}_{t}, \tilde{z}_{t}\right\rangle\right) p_{t}^{0} \\
& +\mathbb{E}_{t} u_{2}^{\prime}\left(w_{2}+\left(p_{t+1}^{0}+y^{s}\right) z_{t}^{0}+\left\langle\delta^{s}, \tilde{z}_{t}\right\rangle\right)\left(p_{t+1}^{0}+y^{s}\right)
\end{aligned}
$$

and

$$
\begin{aligned}
\frac{\partial U_{t}}{\partial \tilde{z}_{t}}=-u_{1}^{\prime}\left(w_{1}\right. & \left.-p_{t}^{0} z_{t}^{0}-\left\langle\tilde{p}_{t}, \tilde{z}_{t}\right\rangle\right) \tilde{p}_{t} \\
& +\mathbb{E}_{t} u_{2}^{\prime}\left(w_{2}+\left(p_{t+1}^{0}+y^{s}\right) z_{t}^{0}+\left\langle\delta^{s}, \tilde{z}_{t}\right\rangle\right) \delta^{s}
\end{aligned}
$$

3.2 Homogeneous agents. Total outside supply of the risky asset is assumed to be constant through time; it is denoted by $\zeta^{0}$. Total supply of each Arrow securities is zero, thus equilibrium prices are found by substituting

$$
p_{t}^{0}=p_{*}^{0}, \quad \tilde{p}_{t}=\tilde{p}_{*}, \quad z_{t}^{0}=\zeta^{0}, \quad \tilde{z}_{t}=0,
$$

into equation (30), yielding

$$
\begin{aligned}
u_{1}^{\prime}\left(w_{1}-p_{*}^{0} \zeta^{0}\right) p_{*}^{0} & =\mathbb{E}_{t} u_{2}^{\prime}\left(w_{2}+\left(p_{*}^{0}+y^{s}\right) \zeta^{0}\right)\left(p_{*}^{0}+y^{s}\right), \\
p_{*}^{j} & =\frac{u_{2}^{\prime}\left(w_{2}+\left(p_{*}^{0}+y^{j}\right) \zeta^{0}\right)}{u_{1}^{\prime}\left(w_{1}-p_{*}^{0} \zeta^{0}\right)} \alpha_{j}, \quad j=1, \cdots, n .
\end{aligned}
$$


If there is a positive outside supply of risky assets $\zeta^{0}>0$, we can locate sufficient conditions for the existence of an equilibrium price. For, if $p_{*}^{0}=0$, the left hand side of (33) equals 0 while the right hand side of the same equation is positive. Likewise, as $p_{*}^{0} \rightarrow w_{1} / \zeta^{0}$, the left hand side increases beyond all positive bounds, while the right hand side remains finite. By the intermediate value theorem, there is at least one $p_{*}^{0}>0$ satisfying (33); we chose one of these, and we refer to it as the fundamental price of the risky asset. Equation (34) then yields the corresponding fundamental prices $\tilde{p}_{*}$ of the Arrow securities.

In the case of zero outside supply of risky assets, equations ((33)) and ((34)) can be solved for the fundamental prices

$$
p_{*}^{0}=\frac{\mathbb{E} y}{\frac{u_{1}^{\prime}\left(w_{1}\right)}{u_{2}^{\prime}\left(w_{2}\right)}-1} \quad \text { and } \quad p_{*}^{j}=\frac{u_{2}^{\prime}\left(w_{2}\right)}{u_{1}^{\prime}\left(w_{1}\right)} \alpha_{j}, \quad j=1, \cdots, n
$$

To have a positive equilibrium price, it is sufficient to have $\mathbb{E} y>0$ and $u_{1}^{\prime}\left(w_{1}\right)>$ $u_{2}^{\prime}\left(w_{2}\right)$. These conditions are not directly obvious. However, if $u_{1}^{\prime}\left(w_{1}\right)<u_{2}^{\prime}\left(w_{2}\right)$, no agent will want to sell the risky asset, for his increase in utility in the first period will never be compensated by the decrease of utility in the second period, no matter how good the price. If the outside supply is non-zero, this argument does not hold since then all agents can hold a positive amount of the asset.

In general there will be multiple equilibria. Indeed there can even be an equilibrium price that is negative if limited liability does not hold. See Brock (1990) for discussion of the role of limited liability in eliminating negative price equilibria and how to construct examples of multiple equilibria.

In the special case of additively separable utility as in (33), differentiation with respect to $p_{*}^{0}$ shows that the term $u_{1}^{\prime}\left(w_{1}-p_{*}^{0} \zeta^{0}\right) p_{*}^{0}$ is increasing in $p_{*}^{0}$ if $u_{1}^{\prime}>0, u_{1}^{\prime \prime}<0$. One can also show that

$$
\begin{aligned}
& \frac{\partial}{\partial p_{*}^{0}}\left(\mathbb{E}_{t} u_{2}^{\prime}\left(w_{2}+\left(p_{*}^{0}+y^{s}\right) \zeta^{0}\right)\left(p_{*}^{0}+y^{s}\right)\right) \\
& =\mathbb{E}_{t} u_{2}^{\prime \prime}\left(w_{2}+\left(p_{*}^{0}+y^{s}\right) \zeta^{0}\right) \zeta^{0}\left(p_{*}^{0}+y^{s}\right)+\mathbb{E}_{t} u_{2}^{\prime}\left(w_{2}+\left(p_{*}^{0}+y^{s}\right) \zeta^{0}\right) \\
& =\mathbb{E}_{t} u_{2}^{\prime}\left(c_{2}^{s}\right)\left(1+\frac{c_{2}^{s} u_{2}^{\prime \prime}\left(c_{2}^{s}\right)}{u_{2}^{\prime}\left(c_{2}^{s}\right)} \frac{\zeta^{0}\left(p_{*}^{0}+y^{s}\right)}{w_{2}+\zeta^{0}\left(p_{*}^{0}+y^{s}\right)}\right),
\end{aligned}
$$

where $c_{2}^{s}=w_{2}+\left(p_{*}^{0}+y^{s}\right) \zeta^{0}$. Notice that the sign of the last expression is the same as the sign of the term in brackets since $u_{2}^{\prime}>0$. Look at the case $w_{2}=0$. The quantity $\left(1+c_{2}^{s} u_{2}^{\prime \prime}\left(c_{2}^{s}\right)\right) / u_{2}^{\prime}\left(c_{2}^{s}\right)$ is just one minus the Arrow-Pratt relative risk aversion measure. Thus, if the Arrow-Pratt relative risk aversion is always greater than one, for $w_{2}=0$, the right hand side of (33) is decreasing so we have a unique positive equilibrium price for this case. 
3.3 Heterogeneous agents. We are primarily interested in conditions for which the equilibrium found above is stable under reinforcement learning of heterogeneous agents, and how this stability depends on the number of Arrow securities. If prices are close to their equilibrium values, demands will be close to their respective equilibrium values. Demands are of the form $z_{h t}^{0}$ and $\tilde{z}_{h t}$; they are combined in the demand vector $z_{h t}=\left(z_{h t}^{0}, \tilde{z}_{h t}\right)$. Also prices are collected in the price vector $p_{t}=\left(p_{t}^{0}, \tilde{p}_{t}\right)$.

Heterogeneity is restricted to the agent's expectation of the future price. As in section 2 we assume that an agent of type $h$ expects this to be

$$
\mathbb{E}_{h t} p_{t+1}^{0}=p_{*}^{0}+f_{h t}=p_{h t+1}^{0} .
$$

As before, $f_{h}$ represents a function of past deviations from the fundamental according to which type $h$ beliefs that prices will deviate from their fundamental benchmark.

3.3.1 Demands. We shall determine the demands of an agent of type $h$. Given a price vector $p$, this agent determines his demands for the risky asset and the Arrow securities by maximizing the utility function

$$
U_{h t}\left(z_{h t}\right)=u_{1}\left(w_{1}-\left\langle p_{t}, z_{h t}\right\rangle\right)+\mathbb{E}_{h t} u_{2}\left(w_{2}+\left(p_{h t+1}^{0}+y^{s}\right) z_{h t}^{0}+\left\langle\delta^{s}, \tilde{z}_{h t}\right\rangle\right)
$$

Denoting consumption of the agent in period $j$ with $c_{h j}$, the second order derivatives of the function $U$ with respect to $z$ read as

$$
\begin{aligned}
\frac{\partial^{2} U_{h t}}{\partial\left(z_{h t}^{0}\right)^{2}} & =u_{1}^{\prime \prime}\left(c_{h 1}\right)\left(p_{t}^{0}\right)^{2}+\mathbb{E}_{h t} u_{2}^{\prime \prime}\left(c_{h 2}^{s}\right)\left(p_{h t+1}^{0}+y^{s}\right)^{2}, \\
\frac{\partial^{2} U_{h t}}{\partial z_{h t}^{0} \partial z_{h t}^{j}} & =u_{1}^{\prime \prime}\left(c_{h 1}\right) p_{t}^{0} p_{t}^{j}+u_{2}^{\prime \prime}\left(c_{h 2}^{j}\right)\left(p_{h t+1}^{0}+y^{j}\right) \alpha_{j}, \\
\frac{\partial^{2} U_{h t}}{\partial\left(z_{h t}^{j}\right)^{2}} & =u_{1}^{\prime \prime}\left(c_{h 1}\right)\left(p_{t}^{j}\right)^{2}+u_{2}^{\prime \prime}\left(c_{h 2}^{j}\right) \alpha_{j}, \\
\frac{\partial^{2} U_{h t}}{\partial z_{h t}^{j} \partial z_{h t}^{k}} & =u_{1}^{\prime \prime}\left(c_{h 1}\right) p_{t}^{j} p_{t}^{k}, \quad \text { if } \quad k \neq j .
\end{aligned}
$$

In particular, the Hessian $H_{z_{h t}} U_{h t}$ is negative definite; hence there is a unique demand vector $z_{h t}=z\left(p_{t}, p_{h t+1}^{0}\right)$ solving the maximization problem, which is differentiable as a function of $p_{t}$.

3.3.2 Fitness. The average realized utilities $u_{h t+1}$ are obtained by replacing in the expression (35) for $U_{h t}$ idiosyncratic expectations $p_{h t+1}^{0}$ of the price of the risky asset by its realization $p_{t+1}^{0}$, taking for the demands $z_{t}$ the realized demands $z_{h t}$ and averaging over all states $s$ :

$$
u_{h t+1}=u_{1}\left(w_{1}-\left\langle p_{t}, z_{h t}\right\rangle\right)+\mathbb{E} u_{2}\left(w_{2}+\left(p_{t+1}^{0}+y\right) z_{h t}^{0}+\left\langle\delta, \tilde{z}_{h t}\right\rangle\right) .
$$


Note that at the beginning of trading period $t$, the most recent price known is $p_{t-1}$, and the most recent realized utility known is $u_{h t-1}$. This utility determines the fraction $n_{h t}$ of traders following type strategy $h$ at the beginning of trading period $t$, through reinforcement learning as discussed in Section 2:

$$
n_{h t}=\mathrm{e}^{\beta u_{h t-1}} / Z_{t}, \quad Z_{t}=\sum_{h=1}^{H} \mathrm{e}^{\beta u_{h t-1}} .
$$

3.3.3 Prices. So far, we have determined demand and fitness of a trader of type $h$. Assume now that there are $H$ trader types in the market. Market clearing requires

$$
\sum_{h=1}^{H} n_{h t} z_{h t}^{0}=\zeta_{0}, \quad \text { and } \quad \sum_{h=1}^{H} n_{h t} \tilde{z}_{h t}=0, \quad j=1, \cdots, n .
$$

There is a large literature on existence of equilibrium prices in incomplete markets; see e.g. Magill and Quinzii (1996) for an overview and the recent discussion in Cass (2006). However, most existence proofs are concerned with homogeneous expectations. Heterogeneous expectations imply that the demands for Arrow securities may move differently for different trader types, which is an additional source of technical difficulties. For an existence proof taking heterogeneous characteristics into account see, e.g., Grandmont and Younes (1972). In order to be self-contained, in the appendix we demonstrate the existence of an equilibrium price vector for these markets with heterogeneous expectations; here we only give an outline of the argument.

Let $P$ denote the set $\left\{p \in \mathbb{R}^{n+1} \mid p_{j}>0\right.$ for $\left.j=0,1, \cdots, n\right\}$ of positive price vectors. We construct a homotopy of the problem from the homogeneous situation where all agents have the same expectations about future prices to the heterogeneous situation we are interested in. It is straightforward to show uniqueness and non-degenerateness of the price equilibrium in the homogeneous case.

We will construct a compact and convex subset $K \subset P$ with piecewise smooth boundary $\partial K$, which is such that along the homotopy, for every $p_{t} \in \partial K$, the aggregate excess demand vector $\bar{z}_{t}\left(p_{t}\right)=\sum_{h=1}^{H} n_{h t} z_{h t}\left(p_{t}\right)$ does not vanish. Then it follows from topological arguments (index theory) that there is a price vector $\hat{p}_{t} \in K$ such that $\bar{z}_{t}\left(\hat{p}_{t}\right)=0$. The details of the proof can be found in section $\mathrm{C}$ of the appendix.

3.4 Dynamics close to a steady state equilibrium. We are mainly interested in the reinforcement learning dynamics within this general equilibrium OG framework. It turns out that a linear approximation of the learning dynamics around a homogeneous steady state equilibrium has essentially the same structure as the evolutionary selection dynamics in the mean-variance asset pricing model with heterogeneous beliefs in Section 2. This result can be derived under the general assumption that utility of agents of type $h$ at time $t$ is of the form

$$
U_{h t}\left(z_{h t}\right)=U\left(z_{h t}, p_{t}, p_{h t+1}^{0}\right),
$$


where $U\left(z_{h t}, p_{t}, p_{h t+1}^{0}\right)$ is strictly concave in $z_{h t}$. In fact, we make the stronger assumption that the Hessian matrix $H_{z} U\left(z, p, p_{+}^{0}\right)$ is negative definite for all arguments. To derive a linear approximation of the price dynamics under reinforcement learning, we will need a quadratic approximation of the utility function. In order to keep notation to a minimum, we shall give the general results about $U$ without time indices, replacing $t+1$ by a mere ' + ', while results for our specification (35) are given with time indices.

Let $p_{t}=p_{*}$ be an homogeneous equilibrium price vector, whose existence we have shown in subsection 3.2 , let moreover $p_{*}^{0}$ be the equilibrium price of the risky asset and let $\tilde{z}_{*}=0$ and $\tilde{z}_{*}^{0}=\zeta^{0}$ be the corresponding steady state demand of type $h$. Demands $z_{h}$ are solutions of the equation

$$
D_{z} U\left(z_{h}, p, p_{h+}^{0}\right)=0 .
$$

As the Hessian matrix $H_{z} U$ is negative definite everywhere, the implicit function theorem yields that

$$
D z_{h}\left(p_{*}, p_{*}^{0}\right)=-\left[H_{z} U\right]^{-1}\left(\begin{array}{ll}
D_{p} D_{z} U & \left.D_{p_{h+}^{0}} D_{z} U\right)
\end{array}\right)
$$

all expressions on the right hand side are evaluated at $\left(z_{*}, p_{*}, p_{*}^{0}\right)$. We expand $U_{h}=$ $U\left(z_{h}, p, p_{h+}^{0}\right)$ around the point $\left(z_{h *}, p_{*}, p_{*}^{0}\right)$, to obtain

$$
\begin{aligned}
U\left(z_{h}, p, p_{h+}^{0}\right)=U & \left(z_{*}, p, p_{h+}^{0}\right)+\left\langle B_{h}, z_{h}-z_{*}\right\rangle \\
& -\frac{1}{2}\left\langle z_{h}-z_{*}, V\left(z_{h}-z_{*}\right)\right\rangle+\mathrm{O}(3) .
\end{aligned}
$$

Here the first term $U\left(z_{*}, p, p_{h+}^{0}\right)$ contains all terms up to second order in $\left(p-p_{*}\right)$ and $\left(p_{h+}^{0}-p_{*}^{0}\right)$, and $\mathrm{O}(3)$ denotes terms $\mathrm{O}\left(\left\|z_{h}-z_{*}\right\|^{\alpha}\left\|p-p_{*}\right\|^{\beta}\left|p_{h+}^{0}-p_{*}^{0}\right|^{\gamma}\right)$ with $\alpha+\beta+\gamma=$ 3 . For the quadratic and linear terms in $z_{h}-z_{*}$ in (39) we introduced

$$
V=-H_{z} U\left(z_{*}, p_{*}, p_{*}^{0}\right)
$$

and

$$
\begin{aligned}
B_{h}= & B\left(p, p_{h+}^{0}\right) \\
= & D_{z} U\left(z_{*}, p_{*}, p_{*}^{0}\right) \\
& \quad+D_{p} D_{z} U\left(z_{*}, p_{*}, p_{*}^{0}\right)\left(p-p_{*}\right)+D_{p_{+}^{0}} D_{z} U\left(z_{*}, p_{*}, p_{*}^{0}\right)\left(p_{h+}^{0}-p_{*}^{0}\right) \\
= & -A\left(p-p_{*}\right)+\left(p_{h+}^{0}-p_{*}^{0}\right) b
\end{aligned}
$$

with $A=-D_{p} D_{z} U\left(z_{*}, p_{*}, p_{*}^{0}\right)$ and $b=D_{p_{+}^{0}} D_{z} U\left(z_{*}, p_{*}, p_{*}^{0}\right)$, and where we used that $D_{z} U\left(z_{*}, p_{*}, p_{*}^{0}\right)=0$. We find consequently that the first order Taylor expansion of $z_{h}$ around $\left(p_{*}, p_{*}^{0}\right)$ reads as

$$
\begin{aligned}
z_{h}\left(p, p_{h+}^{0}\right) & =z_{*}+D z_{h}\left(p_{*}, p_{*}^{0}\right)\left(\begin{array}{c}
p-p_{*} \\
p_{h+}^{0}-p_{*}^{0}
\end{array}\right)+\mathrm{O}\left(\left\|p-p_{*}\right\|^{2}+\left|p_{h+}^{0}-p_{*}^{0}\right|^{2}\right) \\
& =z_{*}+V^{-1} B_{h}+\mathrm{O}\left(\left\|p-p_{*}\right\|^{2}+\left|p_{h+}^{0}-p_{*}^{0}\right|^{2}\right)
\end{aligned}
$$


Notice that the linear part of the demand is of the same form as in the mean-variance asset pricing model in equation (8).

To simplify notation for our utility specification (35), quantities $q_{*}^{s}=p_{*}^{0}+y^{s}, \kappa_{1}=$ $w_{1}-p_{*}^{0} \zeta^{0}$ and $\kappa_{2}^{s}=w_{2}+q_{*}^{s} \zeta^{0}$ are introduced. Instead of prices, we take as basic quantities price deviations $x_{t}=p_{t}-p_{*}$ from the equilibrium values. The vector $B_{h t}$ reads as

$$
\begin{aligned}
B_{h t}= & -u_{1}^{\prime}\left(\kappa_{1}\right)\left(\begin{array}{c}
x_{t}^{0} \\
\tilde{x}_{t}
\end{array}\right)+\mathbb{E}_{t} u_{2}^{\prime}\left(\kappa_{2}\right)\left(\begin{array}{c}
f_{h t} \\
0
\end{array}\right) \\
& +\zeta^{0}\left[u_{1}^{\prime \prime}\left(\kappa_{1}\right) x_{t}^{0}\left(\begin{array}{c}
p_{*}^{0} \\
\tilde{p}_{*}
\end{array}\right)+\mathbb{E}_{t} u_{2}^{\prime \prime}\left(\kappa_{2}\right) f_{h t}\left(\begin{array}{c}
q_{*} \\
\delta
\end{array}\right)\right] .
\end{aligned}
$$

The matrix $V$ reads

$$
V=u_{1}^{\prime \prime}\left(\kappa_{1}\right)\left(\begin{array}{cc}
\left(p_{*}^{0}\right)^{2} & p_{*}^{0} \tilde{p}_{*}^{T} \\
p_{*}^{0} \tilde{p}_{*} & \tilde{p}_{*} \tilde{p}_{*}^{T}
\end{array}\right)+\mathbb{E}_{t} u_{2}^{\prime \prime}\left(\kappa_{2}\right)\left(\begin{array}{cc}
\left(q_{*}\right)^{2} & q_{*} \delta^{T} \\
q_{*} \delta & \delta \delta^{T}
\end{array}\right) .
$$

Note that $\delta \delta^{T}$ is the matrix with elements $\delta_{i} \delta_{j}$ etc.; also note that $V$ is positive definite, due to the differential strict concavity of $u_{1}$ and $u_{2}$.

3.4.1 Market clearing. Market clearing reads as

$$
\sum_{h} n_{h} z_{h}\left(p, p_{h+}^{0}\right)=\left(\begin{array}{c}
\zeta^{0} \\
0
\end{array}\right)
$$

As $z_{h}\left(p_{*}, p_{*}^{0}\right)=z_{*}=\left(\zeta^{0}, 0\right)$ for all types $h,(43)$ is satisfied for the equilibrium price vector $\left(p, p_{h+}^{0}\right)=\left(p_{*}, p_{*}^{0}\right)$. The implicit function theorem implies that $p$ can be solved from (43) for prices close to the fundamental price if the matrix

$$
D_{p} \sum_{h} n_{h} z_{h}\left(p_{*}, p_{*}^{0}\right)=\sum_{h} n_{h} D_{p} z_{h}\left(p_{*}, p_{*}^{0}\right)=-V^{-1} D_{p} D_{z} U\left(z_{*}, p_{*}, p_{*}^{0}\right)
$$

is invertible. Since $V^{-1}$ is known to be invertible, this is equivalent to the condition that $A=-D_{p} D_{z} U\left(z_{*}, p_{*}, p_{*}^{0}\right)$ is invertible. This is an assumption we have to make in the general treatment.

For the specification (35), we have

$$
A=u_{1}^{\prime}\left(\kappa_{1}\right) I-u_{1}^{\prime \prime}\left(\kappa_{1}\right) \zeta^{0}\left(\begin{array}{cccc}
p_{*}^{0} & 0 & \cdots & 0 \\
\vdots & \vdots & & \vdots \\
p_{*}^{n} & 0 & \ldots & 0
\end{array}\right)
$$

and since this matrix is lower triangular with positive diagonal elements, it is certainly invertible.

Consequently, there is a constant $\eta>0$ and a smooth function

$$
\varphi:\left\{p_{h+}^{0}:\left|p_{h+}^{0}-p_{*}^{0}\right|<\eta \text { for all } h\right\} \rightarrow \mathbb{R},
$$


such that the price

$$
p_{t}=\varphi\left(p_{1+}^{0}, \cdots, p_{H+}^{0}\right)
$$

solves equation (43); that is, the price $p$ clears the market.

Making use of the fact that the matrix $V$ is independent of $h$ and of equation (41), market clearing (43) implies

$$
\sum_{h} n_{h} B_{h}=\mathrm{O}\left(\|x\|^{2}+\sum_{h}\left|f_{h}\right|^{2}\right)
$$

Substituting from (40) $B_{h}=-A\left(p-p_{*}\right)+\left(p_{h+}^{0}-p_{*}^{0}\right) b=-A x+f_{h} b$, using the implicit function theorem to solve for $x$ and the invertibility of $A$, this can be rewritten to

$$
x=A^{-1} b \sum_{h} n_{h} f_{h}+\mathrm{O}\left(\sum_{h}\left|f_{h}\right|^{2}\right) .
$$

For our specification it follows, using (42), that

$$
x_{t}^{0}=\frac{\mathbb{E}_{t} u_{2}^{\prime}\left(\kappa_{2}\right)+\zeta^{0} \mathbb{E}_{t} u_{2}^{\prime \prime}\left(\kappa_{2}\right)\left(p_{*}^{0}+y\right)}{u_{1}^{\prime}\left(\kappa_{1}\right)-\zeta^{0} p_{*}^{0} u_{1}^{\prime \prime}\left(\kappa_{1}\right)} \sum_{h} n_{h t} f_{h t}+\mathrm{O}\left(\sum_{h}\left|f_{h t}\right|^{2}\right) .
$$

The coefficient in front of the sum is independent of $t$; by analogy to the mean-variance asset pricing model in section 2 , it will be denoted by $1 / R$. Note that in the case when the outside supply of the risky asset vanishes, i.e. if $\zeta^{0}=0$, then $R$ simplifies to $u_{1}^{\prime}\left(w_{1}\right) / u_{2}^{\prime}\left(w_{2}\right)$ and (46) simplifies to

$$
x_{t}^{0}=\frac{1}{R} \sum_{h} n_{h t} f_{h t}+\mathrm{O}\left(\sum_{h}\left|f_{h t}\right|^{2}\right), \quad \tilde{x}_{t}^{j}=0+\mathrm{O}\left(\sum_{h}\left|f_{h t}\right|^{2}\right) .
$$

In this special case, a linear approximation of the equilibrium price dynamics in the OG setup in (47) yields the same price dynamics as in the asset pricing model (9) in Section 2. In the general case, using (46) and (42), the prices of the Arrow securities are given as

$$
\tilde{x}_{t}=\zeta^{0}\left(\frac{1}{R} \frac{u_{1}^{\prime \prime}\left(\kappa_{1}\right)}{u_{1}^{\prime}\left(\kappa_{1}\right)} \tilde{p}_{*}+\frac{\mathbb{E}_{t} u_{2}^{\prime}\left(\kappa_{2}\right)}{u_{1}^{\prime}\left(\kappa_{1}\right)} \frac{\mathbb{E}_{t} u_{2}^{\prime \prime}\left(\kappa_{2}\right) \delta}{\mathbb{E}_{t} u_{2}^{\prime}\left(\kappa_{2}\right)}\right) \sum_{h} n_{h t} f_{h t}+\mathrm{O}\left(\sum_{h}\left|f_{h t}\right|^{2}\right) .
$$

Note that the evolution of the prices of the Arrow securities is driven by the evolution of the prices of the risky asset. 
3.4.2 Fitness. The fitness $u_{h+}$ of each strategy, which is taken to be average realized utility, can be computed as soon as the realized price $p_{t+1}^{0}$ is known. Introduce the vector

$$
B=B\left(p, p_{+}^{0}\right)
$$

which is the realization of the vector $B_{h}=B\left(p, p_{h+}^{0}\right)$ in (40). Note that $B$ is the same for all types $h$. Using equation (39) for $U$ and equation (41) for $z_{h}$, we can write average realized utility as

$$
\begin{aligned}
u_{h+} & =U\left(z_{h}, p, p_{+}^{0}\right) \\
& =U\left(z_{*}, p, p_{+}^{0}\right)+\left\langle B, z_{h}-z_{*}\right\rangle-\frac{1}{2}\left\langle z_{h}-z_{*}, V\left(z_{h}-z_{*}\right)\right\rangle+\mathrm{O}(3) \\
& =U\left(z_{*}, p, p_{+}^{0}\right)+\left\langle B, V^{-1} B_{h}\right\rangle-\frac{1}{2}\left\langle B_{h}, V^{-1} B_{h}\right\rangle+\mathrm{O}(3) \\
& =U\left(z_{*}, p, p_{+}^{0}\right)+\frac{1}{2}\left\langle B, V^{-1} B\right\rangle-\frac{1}{2}\left\langle B-B_{h}, V^{-1}\left(B-B_{h}\right)\right\rangle+\mathrm{O}(3) .
\end{aligned}
$$

Note that (50) is similar to the fitness expression (12) in the mean-variance asset pricing model in Section 2. Realized utility has a direct interpretation as a measure of the mismatch $B-B_{h}$ between realization and expectation. This is seen by introducing the non-Euclidean vector norm $\|x\|_{V^{-1}}=\sqrt{\left\langle x, V^{-1} x\right\rangle}$ and writing

$$
\begin{aligned}
u_{h+} & =U\left(z_{*}, p, p_{+}^{0}\right)+\frac{1}{2}\left\langle V^{-1} B, B\right\rangle-\frac{1}{2}\left\langle B-B_{h}, V^{-1}\left(B-B_{h}\right)\right\rangle+\mathrm{O}(3) \\
& =C-\frac{1}{2}\left\|B-B_{h}\right\|_{V^{-1}}^{2}+\mathrm{O}(3)
\end{aligned}
$$

here $C$ is the same for all types. We compute, using (40) and (49)

$$
B-B_{h}=\left(x_{+}^{0}-f_{h}\right) b .
$$

and

$$
u_{h+}=C-\frac{1}{2}\left\langle b, V^{-1} b\right\rangle\left(x_{+}^{0}-f_{h}\right)^{2}+\mathrm{O}\left(\sum_{h}\left|f_{h}\right|^{3}\right) .
$$

The fractions are then given as

$$
n_{h t}=\mathrm{e}^{-\beta\left(\frac{1}{2}\left\langle b, V^{-1} b\right\rangle\left(x_{t-1}^{0}-f_{h t-2}\right)^{2}+\mathrm{O}\left(\sum_{k}\left|f_{h t-2}\right|^{3}\right)\right)} / Z_{t},
$$

where $Z_{t}$ is such that $\sum_{h} n_{h t}=1$.

We have for our specification, using (42)

$$
\begin{aligned}
B_{t}= & -u_{1}^{\prime}\left(\kappa_{1}\right)\left(\begin{array}{c}
x_{t}^{0} \\
\tilde{x}_{t}
\end{array}\right)+\mathbb{E}_{t} u_{2}^{\prime}\left(\kappa_{2}^{s}\right)\left(\begin{array}{c}
x_{t+1}^{0} \\
0
\end{array}\right) \\
& +\zeta^{0}\left[u_{1}^{\prime \prime}\left(\kappa_{1}\right) x_{t}^{0}\left(\begin{array}{c}
p_{*}^{0} \\
\tilde{p}_{*}
\end{array}\right)+\mathbb{E}_{t} u_{2}^{\prime \prime}\left(\kappa_{2}^{s}\right) x_{t+1}^{0}\left(\begin{array}{c}
p_{*}^{0}+y^{s} \\
\delta^{s}
\end{array}\right)\right]
\end{aligned}
$$


hence

$$
b=\mathbb{E}_{t} u_{2}^{\prime}\left(\kappa_{2}^{s}\right)\left(\begin{array}{l}
1 \\
0
\end{array}\right)+\zeta^{0} \mathbb{E}_{t} u_{2}^{\prime \prime}\left(\kappa_{2}^{s}\right)\left(\begin{array}{c}
p_{*}^{0}+y^{s} \\
\delta^{s}
\end{array}\right) .
$$

From the matrix lemma 1 and equation (15) it follows as in section 2 that $\left\langle b, V^{-1} b\right\rangle$ increases whenever an Arrow security is added.

3.4.3 Dynamics. We have now derived the price dynamics under reinforcement learning close to the homogeneous expectations equilibrium $p_{*}$, in the OG setup with general utility function of the form (38). The dynamics is given by

$$
\begin{aligned}
x_{t} & =A^{-1} b \sum_{h} n_{h t} f_{h t}+\mathrm{O}\left(\sum_{h}\left|f_{h t}\right|^{2}\right), \\
n_{h t} & =\mathrm{e}^{-\beta\left(\frac{1}{\sigma_{n}^{2}}\left(x_{t-1}^{0}-f_{h t-2}\right)^{2}+\mathrm{O}\left(\sum_{k}\left|f_{h t-2}\right|^{3}\right)\right)} / Z_{t},
\end{aligned}
$$

where, as before, $A=-D_{p} D_{z} U\left(z_{*}, p_{*}, p_{*}^{0}\right), b=D_{p_{+}^{0}} D_{z} U\left(z_{*}, p_{*}, p_{*}^{0}\right), Z_{t}$ is such that $\sum_{h} n_{h t}=1$, and where $\sigma_{n}^{2}=1 /\left\langle b, V^{-1} b\right\rangle$, with $n$ the number of Arrow securities available. Recall from lemma 1 and (17) that

$$
\sigma_{0}^{2}>\sigma_{1}^{2}>\cdots>\sigma_{S-2}^{2}>\sigma_{S-1}^{2}=0
$$

We shall make the assumption that at equilibrium, the belief's biases are small. This means that when prices have been at equilibrium $p_{*}$ in the past, all types forecast a price close to equilibrium $p_{*}$. Precisely, we assume that

$$
f_{h t}\left(x_{t-1}, x_{t-2}, \cdots, x_{t-L}\right)=\varepsilon f_{h}^{0}+\mathrm{O}\left(\left\|\left(x_{t-1}, x_{t-2}, \cdots, x_{t-L}\right)\right\|\right) .
$$

We are now ready to state the main theorem for the OG setup:

THEOREM 2. Consider the OG price dynamics with Arrow securities and reinforcement learning close to a homogeneous expectations equilibrium $p^{*}$, given by (52-53) and beliefs $f_{h}$ as in (55). If $\varepsilon>0$ is sufficiently small, then the following holds.

If $\beta_{0}^{*}$ is the primary bifurcation value in the case without Arrow securities $(n=0)$, that is, if $\beta_{0}^{*}$ is the critical value for which the steady state becomes unstable if there are no Arrow securities, then for almost all dividends $y^{s}$ and probabilities $\alpha_{s}$ the primary bifurcation value $\beta_{n}^{*}$ for the system with $n$ Arrow securities and incomplete markets (i.e. $n<S-1$ ) satisfies

$$
\beta_{n+1}^{*}<\beta_{n}^{*}<\beta_{0}^{*}, \quad 1 \leq n<S-2 .
$$




\section{Proof}

It turns out to be convenient to introduce scaled price deviations

$$
\tilde{x}_{t}=\frac{1}{\varepsilon} x_{t}=\frac{1}{\varepsilon}\left(p_{t}-p_{*}\right),
$$

a scaled intensity of choice parameter $\tilde{\beta}=\varepsilon^{2} \beta$, and scaled beliefs

$$
\tilde{f}_{h t}\left(\tilde{x}_{t-1}, \tilde{x}_{t-2}, \cdots\right)=\frac{1}{\varepsilon} f\left(\varepsilon \tilde{x}_{t-1}, \varepsilon \tilde{x}_{t-2}, \cdots\right)=f_{h}^{0}+\mathrm{O}\left(\left\|\left(\tilde{x}_{t-1}, \tilde{x}_{t-2}, \cdots\right)\right\|\right) .
$$

However, we shall drop all the tildes immediately, taking note of the fact that we are working in local variables close to the steady state equilibrium.

In these local variables, the dynamics take the form

$$
x_{t}=A^{-1} b \sum_{h} f_{h t} \mathrm{e}^{-\left(\beta / \sigma_{n}^{2}\right)\left(x_{t-1}^{0}-f_{h t-2}\right)^{2}} / Z_{t}+\varepsilon \psi\left(x_{t-1}, \cdots ; \beta, \varepsilon\right) .
$$

Here $\psi$ is a smooth function of its arguments, and $Z_{t}=\sum_{h} \mathrm{e}^{-\left(\beta / \sigma_{n}^{2}\right)\left(x_{t-1}^{0}-f_{h t-2}\right)^{2}}$. Let $L$ be the maximum lag length of the forecasting rules $f_{h}$. Introduce the corresponding $(n+1)(L+2)$-dimensional dynamical system

$$
\begin{gathered}
\Phi_{n}\left(x_{t-1}, \cdots, x_{t-L} ; \beta, \varepsilon\right)= \\
\left(\begin{array}{c}
A^{-1} b \sum_{h} f_{h t} \mathrm{e}^{-\left(\beta / \sigma_{n}^{2}\right)\left(x_{t-1}^{0}-f_{h t-2}\right)^{2}} / Z_{t}+\varepsilon \psi\left(x_{t-1}, \cdots ; \beta, \varepsilon\right) \\
x_{t-1} \\
\vdots \\
x_{t-L+1},
\end{array}\right)
\end{gathered}
$$

where $n$ is the number of Arrow securities. Consider first the dominant term of the dynamics in the case of no Arrow securities, that is, equation (57) with $\varepsilon=0$ and $n=$ 0 . Assume that the steady state equilibrium $\left(x_{t-1}, \cdots, x_{t-L+2}\right)=(0, \cdots, 0)$, which corresponds to $p_{t}=p_{*}$ for all $t$, loses stability in a generic bifurcation at $\beta=\beta_{c}^{0}$.

Denoting by $[v]_{k}$ the $k$ 'th component of the vector $v$, we note that

$$
\begin{aligned}
{\left[\Phi_{n}(x ; \beta, 0)\right]_{1} } & =A^{-1} b \sum_{h} f_{h t} \mathrm{e}^{-\frac{\beta}{\sigma_{n}^{2}}\left(x_{t-1}^{0}-f_{h t-2}\right)^{2}} / Z_{t} \\
& =A^{-1} b \sum_{h} f_{h t} \mathrm{e}^{-\frac{\beta\left(\sigma_{0}^{2} / \sigma_{n}^{2}\right)}{\sigma_{0}^{2}}\left(x_{t-1}^{0}-f_{h t-2}\right)^{2}} / Z_{t} \\
& =\left[\Phi_{0}\left(x ; \beta \frac{\sigma_{0}^{2}}{\sigma_{n}^{2}}, 0\right)\right]_{1} .
\end{aligned}
$$

It is clear that if $\beta_{0}^{*}$ is the first bifurcation value for $\Phi_{0}$ as $\beta$ increases towards infinity, then $\Phi_{n}$ will undergo its first bifurcation if

$$
\beta \frac{\sigma_{0}^{2}}{\sigma_{n}^{2}}=\beta_{0}^{*}, \quad \text { that is, if } \beta=\beta_{0}^{*} \frac{\sigma_{n}^{2}}{\sigma_{0}^{2}} \stackrel{\text { def }}{=} \beta_{n}^{*} .
$$


From (54) we infer that

$$
\beta_{0}^{*}>\beta_{1}^{*}>\cdots>\beta_{S-2}^{*}
$$

Now, since the first bifurcation has been assumed to be generic, it will persist if the dynamics are changed by a sufficiently small perturbation. (For these concepts, see Kuznetsov (1995), in particular the discussion following definition 2.14).

In particular, there is $\varepsilon_{0}>0$ and continuous functions $\beta_{*}^{n}(\varepsilon)$, define on $\left[0, \varepsilon_{0}\right]$, such that $\Phi_{n}(x ; \beta, \varepsilon)$ undergoes its first bifurcation at $\beta_{*}^{n}(\varepsilon)$ as $\beta$ increases from 0 to infinity. Though the relation (58) fails to hold in general for $\varepsilon>0$, continuity of the $\beta_{*}^{n}(\varepsilon)$ implies, if necessary after taking $\varepsilon_{0}$ smaller, that

$$
\beta_{*}^{0}(\varepsilon)>\beta_{*}^{1}(\varepsilon)>\cdots>\beta_{*}^{S-2}(\varepsilon) .
$$

for all $0 \leq \varepsilon \leq \varepsilon_{0}$. Note that this equation holds equally well for the original unscaled parameter, as was claimed in the theorem.

3.4.4 Relation between OG-setup and asset pricing model. We have seen that the dynamics of the OG setup close to equilibrium, is similar to the dynamics in the asset pricing model in Section 2. In fact, by choosing an appropriate utility function, the dynamics of the OG-model coincides exactly with the asset pricing dynamics. Consider the utility function

$$
U\left(z_{h}, p, p_{h+}^{0}\right)=-R\left\langle p, z_{h}\right\rangle+p_{h+}^{0} z_{h}^{0}+\left\langle\left(\begin{array}{c}
y \\
\alpha
\end{array}\right), z_{h}\right\rangle-\frac{1}{2}\langle z, V z\rangle,
$$

with $p=\left(p^{0}, \tilde{p}\right)$ and $V=a\left(\begin{array}{cc}\sigma^{2} & \eta^{T} \\ \eta & \Sigma\end{array}\right)$. This is in fact a mean-variance utility function with the Arrow securities, spanning the part of the risk that can be hedged, playing the role of a risk free bond yielding a gross risk free return $R$. We now follow the OG-setup, with utility as in (59), to derive the dynamics around a homogeneous equilibrium.

A homogeneous (fundamental) price equilibrium can be found by solving

$$
0=D_{z} U\left(z_{*}, p_{*}, p_{*}^{0}\right)=-R p_{*}+p_{*}^{0} e_{0}+\left(\begin{array}{c}
y \\
\alpha
\end{array}\right)-\zeta^{0} V e_{0},
$$

where $e_{0}=(1,0, \cdots, 0) \in \mathbb{R}^{n+1}$. Noting that $V e_{0}=\left(\begin{array}{c}a \sigma^{2} \\ a \eta\end{array}\right)$, this is solved by

$$
\begin{aligned}
& p_{*}^{0}=\frac{1}{R-1}\left(y-\zeta^{0} a \sigma^{2}\right) \\
& \tilde{p}_{*}=\frac{1}{R}\left(\alpha-\zeta^{0} a \eta\right)
\end{aligned}
$$


We compute

$$
A=-D_{p} D_{z} U\left(z_{*}, p_{*}, p_{*}^{0}\right)=R \cdot I \quad \text { and } \quad b=-D_{p_{+}^{0}} D_{z} U\left(z_{*}, p_{*}, p_{*}^{0}\right)=e_{0},
$$

where $I$ denotes the $(n+1) \times(n+1)$ identity matrix. We finally find, as in $(52)$, that the dynamics around the homogeneous price equilibrium are given approximately by

$$
x_{t}=A^{-1} b \sum n_{h t} f_{h t}+\mathrm{O}\left(\sum_{h}\left|f_{h t}\right|^{2}\right),
$$

that is

$$
\begin{aligned}
& x_{t}^{0}=\frac{1}{R} \sum n_{h t} f_{h t}+\mathrm{O}\left(\sum_{h}\left|f_{h t}\right|^{2}\right), \\
& \tilde{x}_{t}=0+\mathrm{O}\left(\sum_{h}\left|f_{h t}\right|^{2}\right) .
\end{aligned}
$$

Note that the linear part of these equations coincides exactly with (9). In fact, when utility is quadratic in $z$, as in (59), all higher order terms $O\left(\sum_{h}\left|f_{h t}\right|^{2}\right)$ drop out, and the system exactly coincides with (9).

3.4.5 Example. By way of example, we consider the OG model with agents choosing between the two belief types, near fundamentalists versus trend followers as in $(25-26)$, that is

$$
\begin{aligned}
& f_{1 t}=\varepsilon, \\
& f_{2 t}=x_{t-1}^{0}+g\left(x_{t-1}^{0}-x_{t-2}^{0}\right) .
\end{aligned}
$$

It follows from equations (46) and (48) that close to the homogeneous equilibrium, the dynamics of the system are of the form

$$
\begin{aligned}
R^{j} x_{t}^{j}=n_{1 t} \varepsilon+n_{2 t} & \left(x_{t-1}^{0}+g\left(x_{t-1}^{0}-x_{t-2}^{0}\right)\right) \\
& +\mathrm{O}\left(\varepsilon^{2}+\left\|\left(x_{t-1}^{0}, x_{t-2}^{0}, \cdots\right)\right\|^{2}\right), \quad j=0, \cdots, n
\end{aligned}
$$

where $1 / R^{0}$ and $1 / R^{j}$ are the factors in front of the terms $\sum_{h} n_{h t} f_{h t}$ in equations (46) and (48) respectively.

After introducing scaled variables $x_{t}^{0}=\varepsilon \tilde{x}_{t}^{0}, f_{h t}\left(x_{t-1}^{0}, \cdots\right)=\varepsilon \tilde{f}_{h t}\left(\tilde{x}_{t-1}^{0}, \cdots\right)$ and dropping tildes, these equations take the form

$$
R^{j} x_{t}^{j}=n_{1 t}+n_{2 t}\left(x_{t-1}^{0}+g\left(x_{t-1}^{0}-x_{t-2}^{0}\right)\right)+\mathrm{O}(\varepsilon), \quad j=0, \cdots, n .
$$


The (scaled) version of the fractions, derived from equation (53), taking $\tilde{\beta}=\beta / \varepsilon^{2}$ and dropping the tilde again, is

$$
n_{h t}=\mathrm{e}^{-\beta\left(\frac{1}{\sigma_{n}^{2}}\left(x_{t-1}^{0}-f_{h t-2}\right)^{2}+\mathrm{O}(\varepsilon)\right)} / Z_{t} \quad h=1,2
$$

Note that for the singular scaling $\varepsilon=0$, the system consisting of equation (60) for $j=$ 0 and equation (61) is exactly equal to the (scaled) version of system (28) and (28); therefore, its bifurcation diagram is given by Figure 2 . If $\varepsilon>0$ is sufficiently small, then, by a genericity argument as in the proof of theorem 2 , the bifurcation diagram of the system is but a small perturbation of the diagram given in Figure 2. In particular, the bifurcation values $\beta_{\mathrm{SN}_{1}}, \beta_{\mathrm{Hopf}}$ and $\beta_{\mathrm{SN}_{2}}$ will only shift slightly.

We conclude that, in this example of the OG-setup adding Arrow securities leads to the same bifurcations as illustrated in Figure 4. This example shows that a bifurcation route to multiple steady states and complicated dynamics arises in the OG price dynamics with reinforcement learning.

\section{Information dynamics}

In this section a third model setup is considered, namely a rational expectations asset pricing models with heterogeneous information signals about future dividends, in the spirit of Grossman and Stiglitz (1980). DeFontnouvelle (2000) and Goldbaum (2005, 2006) have studied this type of model with heterogeneous traders and reinforcement learning. The main novel feature here is to investigate how the introduction of additional Arrow securities affects the stability of an asset pricing system with heterogeneous information and reinforcement learning.

A risky asset is traded at market price $p_{t}^{0}$. It pays a dividend $y_{t}^{0}$, which is assumed to be the sum of $N$ independent Gaussian random variables:

$$
y_{t}^{0}=y_{t}^{1}+\cdots,+y_{t}^{N}
$$

with $y_{t}^{k} \sim \mathcal{N}\left(\bar{y}^{k}, \sigma_{k}^{2}\right), 1 \leq k \leq N$. The dividend $y_{t}^{0}$ is hence normally $\mathcal{N}\left(\bar{y}^{0}, \sigma_{0}^{2}\right)$ distributed, with $\bar{y}^{0}=\sum_{k} \bar{y}_{k}$ and $\sigma_{0}^{2}=\sum_{k} \sigma_{k}^{2}$.

We assume that there are tradeable hedging instruments living for one period, which are traded at a price $p_{t}^{k}$ and pay a dividend $y_{t}^{k}$ before perishing. These instruments are only available for $1 \leq k \leq n<N$; they will be termed information assets in the rest of the paper. Moreover, traders can buy a bond at price 1 whose supply is infinitely elastic, paying $R>1$ each period.

Individual traders are indexed by $i$. Before the market opens, each trader $i$ obtains a private signal $s_{i t}^{k}$ about the dividends of the $n$ hedging instruments, as well as a signal 
$\hat{s}_{i t}$ about the non-hedgeable part of the risky asset. These signals are given by

$$
\begin{aligned}
& s_{i t}^{k}=y_{t}^{k}+\varepsilon_{i t}^{k}, \quad k=1, \cdots, n, \\
& \hat{s}_{i t}=\sum_{j=n+1}^{N} y_{t}^{j}+\hat{\varepsilon}_{i t} \equiv \hat{y}_{t}+\hat{\varepsilon}_{i t},
\end{aligned}
$$

where $\hat{y}_{t}=\sum_{j=n+1}^{N} y_{t}^{j}$ is the non-hedgeable part of the dividends, the noise terms $\varepsilon_{i t}^{k}$ and $\hat{\varepsilon}_{i t}$ are normal random variables with mean 0 and variances $\sigma_{i k}^{2}$, $\hat{\sigma}_{i}^{2}$; the $\varepsilon_{i t}^{k}$ are uncorrelated with each other and with the $y_{t}^{k}, 1 \leq k \leq n$.

Trader $i$ buys $z_{i t}^{0}$ units of the risky asset and $z_{i t}^{k}$ units of the $k$ 'th information asset. Introduce price, demand, dividend and signal vectors by

$$
\begin{aligned}
p_{t} & =\left(p_{t}^{0}, p_{t}^{1}, \cdots, p_{t}^{n}\right), \\
z_{i t} & =\left(z_{i t}^{0}, z_{i t}^{1}, \cdots, z_{i t}^{n}\right), \\
y_{t} & =\left(y_{t}^{0}, y_{t}^{1}, \cdots, y_{t}^{n}\right), \quad \text { and } \\
s_{i t} & =\left(s_{i t}^{1}, \cdots, s_{i t}^{n}, \hat{s}_{i t}\right) .
\end{aligned}
$$

Assuming agents to have correct expectations about dividends, that is $\mathbb{E}_{i t} y_{t}^{k}=\bar{y}^{k}$, expected profit of trader $i$ due to trading equals

$$
\begin{aligned}
\pi_{i t} & =\mathbb{E}_{i t}\left(p_{t+1}^{0}\right) z_{i t}^{0}+\sum_{k=0}^{n}\left(\bar{y}^{k}-R p_{t}^{k}\right) z_{i t}^{k} \\
& =\mathbb{E}_{i t}\left(p_{t+1}^{0}\right) z_{i t}^{0}+\left\langle\bar{y}-R p_{t}, z_{i t}\right\rangle .
\end{aligned}
$$

In the model, agents are myopic expected utility maximizers. The expected utility of trader $i$ is taken to be risk-adjusted profits, conditioned on the signals $s_{i t}$ :

$$
U_{i t}=\mathbb{E}\left(\pi_{i t} \mid s_{i t}\right)-\frac{a}{2} \mathbb{V a r}\left(\pi_{i t} \mid s_{i t}\right) .
$$

4.1 Equilibrium price dynamics. We first derive the equilibrium price dynamics.

4.1.1 Conditioning on signals. To evaluate the utility of the agents, the distribution of $\pi_{i t}$ given $s_{i t}$ has to be determined. Recall that all random variables are normally distributed. We need the joint distribution of $\left(\pi_{i t}, s_{i t}\right)$, which, being normal, is characterized by its expected value and its variance-covariance matrix. The expectations of belief of agent $i$ about $\pi_{i t}$ and $s_{i t}$ read as

$$
\begin{aligned}
& \mathbb{E} \pi_{i t}=\mathbb{E}_{i t} p_{t+1}^{0} z_{i t}^{0}+\left\langle\bar{y}-R p_{t}, z_{i t}\right\rangle, \\
& \mathbb{E} s_{i t}^{k}=\bar{y}^{k}, \quad k=1, \cdots, n, \\
& \mathbb{E} \hat{s}_{i t}=\sum_{j=n+1}^{N} \bar{y}^{j} .
\end{aligned}
$$


Recalling that $\hat{y}_{t}=\sum_{j=n+1}^{N} y_{t}^{j}$ and introducing $\overline{\hat{y}}=\mathbb{E} \hat{y}_{t}$ and $\hat{\sigma}^{2}=\mathbb{V a r} \hat{y}_{t}$, the variance-covariance matrix of $\left(\pi_{i t}, s_{i t}\right)$ can be written as

$$
\Sigma_{\pi s}=\left(\begin{array}{ccccc}
\sum_{j=1}^{n}\left(z_{i t}^{0}+z_{i t}^{j}\right)^{2} \sigma_{j}^{2}+\left(z_{i t}^{0}\right)^{2} \hat{\sigma}^{2} & \left(z_{i t}^{0}+z_{i t}^{1}\right) \sigma_{1}^{2} & \cdots & \left(z_{i t}^{0}+z_{i t}^{n}\right) \sigma_{n}^{2} & z_{i t}^{0} \hat{\sigma}^{2} \\
\left(z_{i t}^{0}+z_{i t}^{1}\right) \sigma_{1}^{2} & \sigma_{1}^{2}+\sigma_{i 1}^{2} & \cdots & 0 & 0 \\
\vdots & \vdots & \ddots & \vdots & \vdots \\
\left(z_{i t}^{0}+z_{i t}^{n}\right) \sigma_{n}^{2} & 0 & \cdots & \sigma_{n}^{2}+\sigma_{i n}^{2} & 0 \\
z_{i t}^{0} \hat{\sigma}_{j}^{2} & 0 & \cdots & 0 & \hat{\sigma}^{2}+\hat{\sigma}_{i}^{2}
\end{array}\right)
$$

The following standard lemma can be found for instance in Anderson and Moore (1979), p. 25.

LEMMA 2. Let $X, Y$ be multivariate normal variables, with joint expectation $\left(\mu_{X}, \mu_{Y}\right)$ and variance-covariance matrix $\left(\begin{array}{cc}\Sigma_{X X} & \Sigma_{X Y} \\ \Sigma_{X Y}^{T} & \Sigma_{Y Y}\end{array}\right)$. Then the conditional expectation and variance of $X$ given $Y=y$ is given by

$$
\mathbb{E}(X \mid Y=y)=\mu_{X}+\Sigma_{X Y} \Sigma_{Y Y}^{-1}\left(y-\mu_{Y}\right), \quad \operatorname{Var}(X \mid Y=y)=\Sigma_{X X}-\Sigma_{X Y} \Sigma_{Y Y}^{-1} \Sigma_{X Y}^{T} .
$$

Applying the lemma yields for the conditional expectation

$$
\begin{aligned}
\mathbb{E}\left(\pi_{i t} \mid s_{i t}\right)= & \mathbb{E}_{i t} p_{t+1}^{0} z_{i t}^{0}+\left\langle\bar{y}-R p_{t}, z_{i t}\right\rangle \\
& +\sum_{j=1}^{n}\left(z_{i t}^{0}+z_{i t}^{j}\right) \frac{\sigma_{j}^{2}}{\sigma_{j}^{2}+\sigma_{i j}^{2}}\left(s_{i t}^{j}-\bar{y}^{j}\right)+z_{i t}^{0} \frac{\hat{\sigma}^{2}}{\hat{\sigma}^{2}+\hat{\sigma}_{i}^{2}}\left(\hat{s}_{i t}-\overline{\hat{y}}\right) .
\end{aligned}
$$

The conditional variance of $\pi_{i t}$ reads as

$$
\operatorname{Var}\left(\pi_{i t} \mid s_{i t}\right)=\sum_{j=1}^{n}\left(z_{i t}^{0}+z_{i t}^{j}\right)^{2} \sigma_{j}^{2}\left(1-\frac{\sigma_{j}^{2}}{\sigma_{j}^{2}+\sigma_{i j}^{2}}\right)+\left(z_{i t}^{0}\right)^{2} \hat{\sigma}^{2}\left(1-\frac{\hat{\sigma}^{2}}{\hat{\sigma}^{2}+\hat{\sigma}_{i}^{2}}\right) .
$$

Note that the expressions

$$
\tau_{i}^{j}=\frac{\sigma_{j}^{2}}{\sigma_{j}^{2}+\sigma_{i j}^{2}} \quad \text { and } \quad \hat{\tau}_{i}=\frac{\hat{\sigma}^{2}}{\hat{\sigma}^{2}+\hat{\sigma}_{i}^{2}}
$$

can be viewed as a measure of the precision of the signals: if the variance of the signal $s_{i t}^{j}$ or $\hat{s}_{i t}$ is infinite, the corresponding precision $\tau_{i}^{j}$ or $\hat{\tau}_{i}$ equals zero, while if the variance of the signal vanishes, the signal is perfect and its precision equals unity. We introduce the vector of precisions of agent $i, \tau_{i}=\left(\tau_{i}^{1}, \cdots, \tau_{i}^{n}\right)$. 
4.1.2 Demands. The expected utility $U_{i t}$ of agent $i$, given by (64), is strictly concave in the demands $z_{i t}$. Its maximum is a solution of the first order necessary conditions. Vanishing of the first order derivatives of the utilities with respect to the demands leads to

$$
\begin{aligned}
0=\frac{\partial U_{i t}}{\partial z_{i t}^{0}}=\mathbb{E}_{i t} p_{t+1}^{0}+\bar{y}^{0} & -R p_{t}^{0}+\sum_{j=1}^{n} \tau_{i}^{j}\left(s_{i t}^{j}-\bar{y}^{j}\right)+\hat{\tau}_{i}\left(\hat{s}_{i t}-\overline{\hat{y}}\right) \\
& -a\left(\sum_{j=1}^{n}\left(z_{i t}^{0}+z_{i t}^{j}\right) \sigma_{j}^{2}\left(1-\tau_{i}^{j}\right)+z_{i t}^{0} \hat{\sigma}^{2}\left(1-\hat{\tau}_{i}\right)\right)
\end{aligned}
$$

and

$$
0=\frac{\partial U_{i t}}{\partial z_{i t}^{k}}=\bar{y}^{k}-R p_{t}^{k}+\tau_{i}^{k}\left(s_{i t}^{k}-\bar{y}_{t}^{k}\right)-a\left(z_{i t}^{0}+z_{i t}^{k}\right) \sigma_{k}^{2}\left(1-\tau_{i}^{k}\right) .
$$

Solving the last equation for $z_{i t}^{k}$ yields the demand for the $k$ 'th information asset by agent $i$ :

$$
z_{i t}^{k}=-z_{i t}^{0}+\frac{\bar{y}^{k}-R p_{t}^{k}+\tau_{i}^{k}\left(s_{i t}^{k}-\bar{y}^{k}\right)}{a \sigma_{k}^{2}\left(1-\tau_{i}^{k}\right)} .
$$

Substituting this expression in the first equation and solving for $z_{i t}^{0}$ yields the demand for the risky asset by trader $i$

$$
\begin{aligned}
z_{i t}^{0} & =\frac{1}{a \hat{\sigma}^{2}\left(1-\hat{\tau}_{i}\right)}\left(\mathbb{E}_{i t} p_{t+1}^{0}+\bar{y}^{0}-R p_{t}^{0}-\sum_{j=1}^{n}\left(\bar{y}^{j}-R p_{t}^{j}\right)+\hat{\tau}_{i}\left(\hat{s}_{i t}-\overline{\hat{y}}\right)\right) \\
& =\frac{1}{a \hat{\sigma}^{2}\left(1-\hat{\tau}_{i}\right)}\left(\mathbb{E}_{i t} p_{t+1}^{0}+\overline{\hat{y}}+\hat{\tau}_{i}\left(\hat{s}_{i t}-\overline{\hat{y}}\right)-R\left(p_{t}^{0}-\sum_{j=1}^{n} p_{t}^{j}\right)\right) .
\end{aligned}
$$

These expressions show that demand increases when the agent receives positive signals and signal precision increases. More precisely, suppose that agent $i$ obtains a positive signal about the $\mathrm{k}$-th information asset, i.e. suppose $s_{i t}^{k}-\bar{y}^{k}>0$. Then the demand $z_{i t}^{k}$ for the $\mathrm{k}$-th information asset increases when the signal precision $\tau_{i}^{k}$ increases. Similarly, suppose that agent $i$ obtains a positive signal about the nonhedgeable part of the dividends, i.e. suppose $\hat{s}_{i t}-\overline{\hat{y}}>0$. Then the demand for the risky asset $z_{i t}^{0}$ increases when the precision $\hat{\tau}_{i}$ about the non-hedgeable part of the dividends increases. Obviously, the demand for the risky asset also increases when the price of one of the Arrow securities increases. Finally, notice that when the precision $\tau_{i}^{k}$ of the signal increases and approaches 1 , the demand for the $k$-the information asset tends to (minus) infinity, while when the precision $\hat{\tau}_{i}$ increases and approaches 1 , the demand $z_{t}^{0}$ for the risky asset tends to (minus) infinity. 
4.1.3 Equilibrium. Assume that the individual traders, indexed by $i$, can be divided into $H$ types, indexed by $h=h(i)$. A trader type is characterized by its belief $\mathbb{E}_{h t} p_{t+1}^{0}$ about the future asset price, as well as by the precisions $\tau_{h}^{k}$ and $\hat{\tau}_{h}$ of the signals it gets. If the total number of traders is equal to $M$, we denote by $n_{h t}$ the fraction of traders of type $h$ at time $t$; that means, at time $t$ there will be $M n_{h t}$ traders of type $h$ in the market. If the outside supply of risky assets per trader is given by $\zeta^{0}$, using (68) and (63) equilibrium of supply and aggregate demand for the risky asset, $M \zeta^{0}=\sum_{i} z_{i t}^{0}$, is equivalent to

$$
\begin{array}{r}
M \zeta^{0} a \hat{\sigma}^{2}=M \sum_{h} \frac{n_{h t}}{\left(1-\hat{\tau}_{h}\right)}\left(\mathbb{E}_{h t} p_{t+1}^{0}+\overline{\hat{y}}-R\left(p_{t}^{0}-\sum_{k=1}^{n} p_{t}^{k}\right)\right) \\
+M \sum_{h} n_{h t} \frac{\hat{\tau}_{h}}{1-\hat{\tau}_{h}}\left(\hat{y}_{t}-\overline{\hat{y}}\right)+\sum_{i} \frac{\hat{\tau}_{h(i)} \hat{\varepsilon}_{i t}}{1-\hat{\tau}_{h(i)}} .
\end{array}
$$

In (69), the fractions appear to be weighted by the factor $\left(1-\hat{\tau}_{h}\right)^{-1}$. Introduce weighted fractions $\hat{n}_{h t}$ by introducing $\hat{w}_{h t}=n_{h t} /\left(1-\hat{\tau}_{h}\right), \hat{W}_{t}=\sum_{h} \hat{w}_{h t}$, and setting $\hat{n}_{h t}=\hat{w}_{h t} / \hat{W}_{t}$. This means that types with high precision get relatively higher weights $\hat{n}_{h t}$. Also introduce market precision

$$
\langle\hat{\tau}\rangle=\sum_{h} \hat{n}_{h t} \hat{\tau}_{h}=\frac{\sum_{h} \frac{n_{h t}}{1-\hat{\tau}_{h}} \hat{\tau}_{h}}{\sum_{h} \frac{n_{h t}}{1-\hat{\tau}_{h}}}
$$

Notice that market precision $\langle\hat{\tau}\rangle$ is time dependent, since the fractions $n_{h t}$ are time dependent, but we will not use a time index to keep notation as simple as possible. Using this notation, and dividing by $M \hat{W}_{t}$ the equilibrium equation (69) can be rewritten as

$$
\begin{aligned}
\frac{\zeta^{0} a \hat{\sigma}^{2}}{\hat{W}_{t}}=\sum_{h} \hat{n}_{h t}\left(\mathbb{E}_{h t} p_{t+1}^{0}+\overline{\hat{y}}-R\left(p_{t}^{0}-\sum_{k=1}^{n} p_{t}^{k}\right)\right) \\
\quad+\sum_{h} \hat{n}_{h t} \hat{\tau}_{h}\left(\hat{y}_{t}-\overline{\hat{y}}\right)+\frac{1}{M \hat{W}_{t}} \sum_{i} \frac{\hat{\tau}_{h(i)} \hat{\varepsilon}_{i t}}{1-\hat{\tau}_{h(i)}} .
\end{aligned}
$$

We let the number of agents $M$ go to infinity, while keeping the type fractions $n_{h t}$ fixed. Applying the law of large numbers yields (under reasonable conditions, see Hellwig (1980)) that the last term tends to zero, i.e.

$$
\frac{\sum_{i} \hat{\tau}_{h(i)} \hat{\varepsilon}_{i} /\left(1-\hat{\tau}_{h(i)}\right)}{M \sum_{h} n_{h t} /\left(1-\hat{\tau}_{h(i)}\right)} \rightarrow 0 .
$$

We therefore obtain

$$
p_{t}^{0}-\sum_{j=1}^{n} p_{t}^{j}=\frac{\overline{\hat{y}}}{R}+\langle\hat{\tau}\rangle \frac{\hat{y}_{t}-\overline{\hat{y}}}{R}+\frac{1}{R} \sum_{h=1}^{H} \hat{n}_{h t} \mathbb{E}_{h t} p_{t+1}^{0}-\frac{a \hat{\sigma}^{2} \zeta^{0}}{R \hat{W}_{t}} .
$$


Similarly, using (67) and (63) market clearing for the information assets, $\sum_{i} z_{i t}^{k}=0$, yields

$$
\begin{aligned}
0 & =-M \sum_{h} n_{h t} z_{h t}^{0}+M \sum_{h} n_{h t} \frac{\bar{y}^{k}-R p_{t}^{k}+\tau_{h}^{k}\left(y_{t}^{k}-\bar{y}^{k}\right)}{a \sigma_{k}^{2}\left(1-\tau_{h}^{k}\right)}+\sum_{i} \frac{\tau_{h(i)}^{k} \varepsilon_{i t}}{a \sigma_{k}^{2}\left(1-\tau_{h(i)}\right)} \\
& =-M \zeta^{0}+M \sum_{h} \frac{n_{h t}}{a \sigma_{k}^{2} 1-\tau_{h}^{k}}\left(\bar{y}^{k}-R p_{t}^{k}+\tau_{h}^{k}\left(y_{t}^{k}-\bar{y}^{k}\right)\right)+\sum_{i} \frac{\tau_{h(i)}^{k} \varepsilon_{i t}}{a \sigma_{k}^{2}\left(1-\tau_{h(i)}\right)} .
\end{aligned}
$$

Setting $w_{h t}^{k}=n_{h t} /\left(1-\tau_{h}^{k}\right), W_{t}^{k}=\sum_{h} w_{h t}^{k}, \hat{n}_{h t}^{k}=w_{h t}^{k} / W_{t}^{k}$ and dividing by $M W_{t}^{k}$ we get

$$
\frac{a \sigma_{k}^{2} \zeta^{0}}{W_{t}^{k}}=\sum_{h} \hat{n}_{h t}\left(\bar{y}^{k}-R p_{t}^{k}\right)+\sum_{h} \hat{n}_{h t} \tau_{h}^{k}\left(y_{t}^{k}-\bar{y}^{k}\right)+\sum_{i} \frac{\tau_{h(i)}^{k} \varepsilon_{i t} /\left(1-\tau_{h(i)}\right)}{M W_{t}^{k}}
$$

Introduce the market precision for the $k$-th information asset as

$$
\left\langle\tau^{k}\right\rangle=\sum_{h} \hat{n}_{h t} \tau_{h}^{k}=\frac{\sum_{h} \frac{n_{h t}}{1-\tau_{h}^{k}} \tau_{h}^{k}}{\sum_{h} \frac{n_{h t}}{1-\tau_{h}^{k}}}
$$

As above, letting the number of agents $M$ go to infinity, while keeping the type fractions $n_{h t}$ fixed and applying the law of large numbers yields under reasonable conditions that the last term tends to zero, that is

$$
\frac{\sum_{i} \tau_{h(i)}^{k} \hat{\varepsilon}_{i} /\left(1-\tau_{h(i)}^{k}\right)}{M \sum_{h} n_{h t} /\left(1-\tau_{h(i)}^{k}\right)} \rightarrow 0
$$

We obtain the price of the $k$-th information asset:

$$
p_{t}^{k}=\frac{\bar{y}^{k}}{R}+\left\langle\tau^{k}\right\rangle \frac{y_{t}^{k}-\bar{y}^{k}}{R}-\frac{a \sigma_{k}^{2} \zeta^{0}}{R W_{t}^{k}} .
$$

Combining these equations (75) and (71) yields the equilibrium price for the risky asset

$$
\begin{aligned}
R p_{t}^{0}=\bar{y}^{0}+\sum_{k=1}^{n} & \left\langle\tau^{k}\right\rangle\left(y_{t}^{k}-\bar{y}^{k}\right)+\langle\hat{\tau}\rangle\left(\hat{y}_{t}-\overline{\hat{y}}\right) \\
& +\sum_{h=1}^{H} \hat{n}_{h t} \mathbb{E}_{h t} p_{t+1}^{0}-\zeta^{0}\left(\frac{1}{\hat{W}_{t}}+\sum_{j=1}^{n} \frac{1}{W_{t}^{j}}\right) .
\end{aligned}
$$


4.2 The model with a short lived asset and reinforcement learning. The pricing equation (76) contains a term $\hat{n}_{h t} \mathbb{E}_{h t} p_{t+1}^{0}$ representing heterogeneous beliefs about the future price of the risky asset. One could study this model with heterogeneous beliefs in a way similar to the mean-variance asset pricing model in Section 2 or the OG-model in Section 3. Here, we are mainly interested in the information dynamics under reinforcement learning and how the introduction of Arrow securities affects the information dynamics. Therefore we focus on the simplest case where the risky asset is short lived and perishes after one period so that $p_{t+1}^{0}=0$. As an example, one may think of a future on an agricultural commodity, such as corn or soybeans. In the case of a short lived asset heterogeneous beliefs about the future price of the asset does not play any role, and agents are only heterogeneous in their information signals about future dividends. Without loss of generality, we also assume zero outside supply of the risky asset, i.e. $\zeta^{0}=0 .{ }^{1}$ In this simple setup, the equilibrium pricing equation (76) simplifies to

$$
R p_{t}^{0}=\bar{y}^{0}+\sum_{k=1}^{n}\left\langle\tau^{k}\right\rangle\left(y_{t}^{k}-\bar{y}^{k}\right)+\langle\hat{\tau}\rangle\left(\hat{y}_{t}-\overline{\hat{y}}\right) .
$$

4.2.1 General setup. In this subsection we derive general expressions for expected utility and average realized utility, for each type of agents. Each type $h$ is characterized by a precision vector $\left(\tau_{h}^{1}, \cdots, \tau_{h}^{n} ; \hat{\tau}_{h}\right)$. To simplify notation, we drop the time index below. Introduce

$$
\delta^{k}=y^{k}-\bar{y}^{k}, \quad \hat{\delta}=\hat{y}-\overline{\hat{y}} .
$$

Recall from (64), (65) and (66) that expected utility of agent type $h$ is given by

$$
\begin{aligned}
U_{h}= & \sum_{k=0}^{n}\left(\bar{y}^{k}-R p^{k}\right) z_{h}^{k}+\sum_{k=1}^{n} \tau_{h}^{k}\left(\delta^{k}+\varepsilon_{h}^{k}\right)\left(z_{h}^{0}+z_{h}^{k}\right)+\hat{\tau}_{h}\left(\hat{\delta}+\hat{\varepsilon}_{h}\right) z_{h}^{0} \\
& -\frac{a}{2}\left(\sum_{k=1}^{n} \sigma_{k}^{2}\left(1-\tau_{h}^{k}\right)\left(z_{h}^{0}+z_{h}^{k}\right)^{2}+\hat{\sigma}^{2}\left(1-\hat{\tau}_{h}\right)\left(z_{h}^{0}\right)^{2}\right)
\end{aligned}
$$

As before, we can write this as $U_{h}=\left\langle B_{h}, z\right\rangle-\frac{1}{2}\left\langle z, V_{h} z\right\rangle$, where

$$
B_{h}=\left(\begin{array}{c}
\bar{y}^{0}-R p^{0}+\sum_{k=1}^{n}\left(\delta^{k}+\varepsilon_{h}^{k}\right) \tau_{h}^{k}+\left(\hat{\delta}+\hat{\varepsilon}_{h}\right) \hat{\tau}_{h} \\
\bar{y}^{1}-R p^{1}+\left(\delta^{1}+\varepsilon_{h}^{1}\right) \tau_{h}^{1} \\
\vdots \\
\bar{y}^{n}-R p^{n}+\left(\delta^{n}+\varepsilon_{h}^{n}\right) \tau_{h}^{n}
\end{array}\right)
$$

\footnotetext{
${ }^{1}$ For a positive outside supply the last term in eq. (76) could simply be included in the dividend term $\bar{y}^{0}$, which would then be termed a risk adjusted dividend.
} 
and

$$
V_{h}=a\left(\begin{array}{cccc}
\sum_{k=1}^{n} \sigma_{k}^{2}\left(1-\tau_{h}^{k}\right)+\hat{\sigma}^{2}\left(1-\hat{\tau}_{h}\right) & \sigma_{1}^{2}\left(1-\tau_{h}^{1}\right) & \cdots & \sigma_{n}^{2}\left(1-\tau_{h}^{n}\right) \\
\sigma_{1}^{2}\left(1-\tau_{h}^{1}\right) & \sigma_{1}^{2}\left(1-\tau_{h}^{1}\right) & \cdots & 0 \\
\vdots & \vdots & \ddots & \vdots \\
\sigma_{n}^{2}\left(1-\tau_{h}^{n}\right) & 0 & \ldots & \sigma_{n}^{2}\left(1-\tau_{h}^{n}\right)
\end{array}\right)
$$

The inverse of $V_{h}$ is given by

$$
V_{h}^{-1}=\frac{1}{a \hat{\sigma}^{2}\left(1-\hat{\tau}_{h}\right)}\left(\begin{array}{ccccc}
1 & -1 & -1 & \ldots & -1 \\
-1 & 1+\frac{\hat{\sigma}^{2}\left(1-\hat{\tau}_{h}\right)}{\sigma_{1}^{2}\left(1-\tau_{h}^{1}\right)} & 1 & \ldots & 1 \\
-1 & 1 & \ddots & \ddots & \vdots \\
\vdots & \vdots & \ddots & \ddots & 1 \\
-1 & 1 & \ldots & 1 & 1+\frac{\hat{\sigma}^{2}\left(1-\hat{\tau}_{h}\right)}{\sigma_{n}^{2}\left(1-\tau_{h}^{n}\right)}
\end{array}\right)
$$

Optimal demands are, as before, given by

$$
z_{h}=V_{h}^{-1} B_{h}=\left(\begin{array}{c}
\frac{\overline{\hat{y}}-R\left(p^{0}-\sum_{k} p^{k}\right)+\hat{\tau}_{h}\left(\hat{\delta}+\hat{\varepsilon}_{h}\right)}{a \hat{\sigma}^{2}\left(1-\hat{\tau}_{h}\right)} \\
-\frac{\overline{\hat{y}}-R\left(p^{0}-\sum_{k} p^{k}\right)+\hat{\tau}_{h}\left(\hat{\delta}+\hat{\varepsilon}_{h}\right)}{a \hat{\sigma}^{2}\left(1-\hat{\tau}_{h}\right)}+\frac{\bar{y}^{1}-R p^{1}+\tau_{h}^{1}\left(\delta^{1}+\varepsilon_{h}^{1}\right)}{a \sigma_{1}^{2}\left(1-\tau_{h}^{1}\right)} \\
\vdots \\
-\frac{\overline{\hat{y}}-R\left(p^{0}-\sum_{k} p^{k}\right)+\hat{\tau}_{h}\left(\hat{\delta}+\hat{\varepsilon}_{h}\right)}{a \hat{\sigma}^{2}\left(1-\hat{\tau}_{h}\right)}+\frac{\bar{y}^{n}-R p^{n}+\tau_{h}^{n}\left(\delta^{n}+\varepsilon_{h}^{n}\right)}{a \sigma_{n}^{2}\left(1-\tau_{h}^{n}\right)}
\end{array}\right) .
$$

Using the pricing equations (71) and (75), realized demands read as

$$
z_{h}=\left(\begin{array}{c}
\frac{\hat{\tau}_{h}\left(\hat{\delta}+\hat{\varepsilon}_{h}\right)-\langle\hat{\tau}\rangle \hat{\delta}}{a \hat{\sigma}^{2}\left(1-\hat{\tau}_{h}\right)} \\
-\frac{\hat{\tau}_{h}\left(\hat{\delta}+\hat{\varepsilon}_{h}\right)-\langle\hat{\tau}\rangle \hat{\delta}}{a \hat{\sigma}^{2}\left(1-\hat{\tau}_{h}\right)}+\frac{\tau_{h}^{1}\left(\delta^{1}+\varepsilon_{h}^{1}\right)-\left\langle\tau^{1}\right\rangle \delta^{1}}{a \sigma_{1}^{2}\left(1-\tau_{h}^{1}\right)} \\
\vdots \\
-\frac{\hat{\tau}_{h}\left(\hat{\delta}+\hat{\varepsilon}_{h}\right)-\langle\hat{\tau}\rangle \hat{\delta}}{a \hat{\sigma}^{2}\left(1-\hat{\tau}_{h}\right)}+\frac{\tau_{h}^{n}\left(\delta^{n}+\varepsilon_{h}^{n}\right)-\left\langle\tau^{n}\right\rangle \delta^{n}}{a \sigma_{n}^{2}\left(1-\tau_{h}^{n}\right)}
\end{array}\right) .
$$

From this, we obtain the realized utility

$$
u_{h}=\frac{1}{2}\left\langle B_{h}, V_{h}^{-1} B_{h}\right\rangle=\frac{\left[\left(\hat{\tau}_{h}-\langle\hat{\tau}\rangle\right) \hat{\delta}+\hat{\tau}_{h} \hat{\varepsilon}_{h}\right]^{2}}{a \hat{\sigma}^{2}\left(1-\hat{\tau}_{h}\right)}+\sum_{k=1}^{n} \frac{\left[\left(\tau_{h}^{k}-\left\langle\tau^{k}\right\rangle\right) \delta^{k}+\tau_{h}^{k} \varepsilon_{h}^{k}\right]^{2}}{a \sigma_{k}^{2}\left(1-\tau_{h}^{k}\right)}
$$


We note that

$$
\mathbb{E} \frac{\left(\tau_{h}^{k} \varepsilon_{h}^{k}\right)^{2}}{a \sigma_{k}^{2}\left(1-\tau_{h}^{k}\right)}=\frac{\left(\tau_{h}^{k}\right)^{2} \sigma_{h k}^{2}}{a \sigma_{k}^{2}\left(1-\tau_{h}^{k}\right)}=\frac{\sigma_{k}^{4}}{\left(\sigma_{k}^{2}+\sigma_{h k}^{2}\right)^{2}} \frac{\sigma_{h k}^{2}}{\sigma_{k}^{2}} \frac{1}{1-\frac{\sigma_{k}^{2}}{\sigma_{k}^{2}+\sigma_{h k}^{2}}}=\tau_{h}^{k},
$$

and a similar equality holds for the hatted variables. The average realized utility is now easily seen to be equal to

$$
\mathbb{E} u_{h}=\frac{\left(\hat{\tau}_{h}-\langle\hat{\tau}\rangle\right)^{2}}{a\left(1-\hat{\tau}_{h}\right)}+\sum_{k=1}^{n} \frac{\left(\tau_{h}^{k}-\left\langle\tau^{k}\right\rangle\right)^{2}}{a\left(1-\tau_{h}^{k}\right)}+\frac{1}{a}\left(\hat{\tau}_{h}+\sum_{k} \tau_{h}^{k}\right),
$$

with market precisions $\langle\hat{\tau}\rangle$ and $\left\langle\tau^{k}\right\rangle$ as defined as in (70) and in (74). The last term reflects that average realized utility will be higher when the precision of the signals is higher. Note however that the first two terms reflect that average realized utility is high if the difference between type precision and market precision is large, and this effect becomes stronger when the precision is higher and approaches 1 . This means that it pays to deviate from average market precision, especially for better informed types. Moreover, the distribution of the information assets themselves do not enter into this utility other than by the precisions: to the traders they are sources of randomness on which they have a certain amount of information, measured by the precisions $\tau_{h}^{k}$ and $\hat{\tau}_{h}$.

Finally, similar to sections 2 and 3 the new fractions are determined by reinforcement learning based on past average realized utilities according to the familiar discrete choice probabilities:

$$
n_{h t}=\frac{\mathrm{e}^{\beta \mathbb{E} u_{h, t-1}}}{Z_{t}}, \quad Z_{t}=\sum_{h} \mathrm{e}^{\beta \mathbb{E} u_{h, t-1}} .
$$

4.3 Adding more Arrow securities. We now return to the main theme of this paper within the current setup with heterogeneous information signals: what happens to the information dynamics under reinforcement learning, when more Arrow securities are added to the market?

4.3.1 A general result with $H$ information types. Traders are heterogeneous in the precision of their signals across markets, but the current setup still allows for a wide class of heterogeneous precision vectors specifying the precisions in the risky asset market and each of the $n$ Arrow security markets. Consider a simple structure of informational heterogeneity, where each type $h$ has the same precision in each market, that is,

$$
\tau_{h}^{1}=\ldots=\tau_{h}^{n}=\hat{\tau}_{h}=\tau_{h}, \quad h=1, \cdots, H .
$$


With this information structure, better informed traders have better information for all markets. We also assume that better information is more costly. Let the information precisions of the $H$ types be ranked according to

$$
1>\tau_{1}>\tau_{2}>\cdots>\tau_{H} \geq 0 .
$$

Higher costs for better informed types means

$$
C_{1}>C_{2}>\cdots>C_{H} \geq 0 .
$$

We assume that the costs of information precision $\tau^{h}$ is the same for each market, so that the total costs for type $h$ is proportional to the number of markets. Using (79), net utilities are given by

$$
\mathbb{E} u_{h}=\frac{n+1}{a}\left(\tau_{h}+\frac{\left(\tau_{h}-<\tau>\right)^{2}}{1-\tau_{h}}-C_{h}\right) .
$$

Because of the multiplicative factor $n+1$, it is immediately clear that adding another information asset to the system will magnify differences in averaged realized utility and therefore may destabilize the system. Hence, we have shown the following:

THEOREM 3. Consider the price dynamics in (77) with reinforcement learning in (80) based on average realized utility in (79), information heterogeneity structure as in (81) and (82), and costs of information as in (83). If $\beta_{0}^{*}$ is the primary bifurcation value in the case without Arrow securities, that is, if $\beta_{0}^{*}$ is the critical value for which the steady state becomes unstable if there are no Arrow securities, then the primary bifurcation value $\beta_{n}^{*}$ for the system with $n$ Arrow securities and incomplete markets (i.e. $n<N$ ) satisfies

$$
\beta_{n+1}^{*}<\beta_{n}^{*}<\beta_{0}^{*}, \quad 1 \leq n<N .
$$

This implies that for the information dynamics with heterogeneous information signals, we have a similar result that in the presence of more Arrow securities the primary bifurcation to instability occurs earlier. Adding an Arrow security to the system causes the primary bifurcation in the information dynamics to occur earlier.

4.3.2 Two type example. In this subsection, we present an explicit example in which a bifurcation occurs when more Arrow securities are added to the market. Consider the situation where there are only two types of traders, for which $\tau_{h}^{j}=\hat{\tau}_{h}=\tau_{h}$, $h=1,2$. We assume that type 1 traders are better informed, i.e., $0 \leq \tau_{2}<\tau_{1}<1$, and that better information is more costly, i.e. $C=C_{1}-C_{2} \geq 0$.

Market precisions $\left\langle\tau^{j}\right\rangle$ and $\langle\hat{\tau}\rangle$ at time $t$ are equal to

$$
\langle\tau\rangle=\frac{\frac{n_{1 t} \tau_{1}}{1-\tau_{1}}+\frac{\left(1-n_{1 t}\right) \tau_{2}}{1-\tau_{2}}}{\frac{n_{1 t}}{1-\tau_{1}}+\frac{1-n_{1 t}}{1-\tau_{2}}}=\frac{n_{1 t}\left(\tau_{1}-\tau_{2}\right)+\tau_{2}\left(1-\tau_{1}\right)}{n_{1 t}\left(\tau_{1}-\tau_{2}\right)+\left(1-\tau_{1}\right)}
$$


Note that, as the fraction $n_{1}$ of better informed increases from 0 to 1 , market precision $\langle\tau\rangle$ increases from $\tau_{2}$ to $\tau_{1}$. Using average realized net utility in (84), the difference in utility is equal to

$$
\mathbb{E} u_{1}-\mathbb{E} u_{2}=\frac{(n+1)}{a} \frac{\tau_{1}-\tau_{2}}{\left(1-\tau_{1}\right)\left(1-\tau_{2}\right)}\left((\langle\tau\rangle-1)^{2}-C\right),
$$

where $n$ is the number of Arrow securities as before and $C=C_{1}-C_{2} \geq 0$. Notice that when better information comes at no costs, i.e, if $C=0$, then the better informed type 1 will always outperform the lesser informed type 2 . Using (80) the fraction of type 1 is given by

$$
n_{1, t}=\frac{\mathrm{e}^{\beta \mathbb{E} u_{1, t-1}}}{\mathrm{e}^{\beta \mathbb{E} u_{1, t-1}}+\mathrm{e}^{\beta \mathbb{E} u_{2, t-1}}},
$$

Re-writing this expression, we obtain

$$
n_{1, t}=\frac{1}{1+\mathrm{e}^{-\beta\left(\mathbb{E} u_{1, t-1}-\mathbb{E} u_{2, t-1}\right)}}=f_{\tau_{1}, \tau_{2}, \beta, C, n}\left(n_{1 t}\right) .
$$

Equations (86), (87) and (88) together fully specify the dynamics of the fraction of better informed traders. The graph of the one-dimensional (1-D) map $f_{\tau_{1}, \tau_{2}, \beta, C, n}$ is illustrated in Figure 5. The reader may easily check that $f_{\tau_{1}, \tau_{2}, \beta, C, n}$ is always decreasing. The Figure illustrates an example with $\tau_{1}=0.35, \tau_{2}=0, C_{1}=0.5, C_{2}=0$. The information dynamics converge to a steady state when there are no Arrow securities; this steady state will be destabilized as more Arrow securities are introduced in the market. When the system is unstable, it converges to a stable 2-cycle, with up and down oscillations. Adding Arrow securities thus leads to a period doubling bifurcation in the information dynamics. Along the 2-cycle, agents switch between buying better but costly information and a free riding on cheap information strategy.

\section{Concluding Remarks}

Developments in the financial sector have created many more possibilities for hedging risk. It has been argued recently, e.g., by Rajan (2005), that under some conditions markets may be exposed to more financial-sector turmoil than in the past. We have formalized this idea in three different model settings, a simple mean-variance asset pricing framework, a general equilibrium OG-model and a noisy rational expectations model with heterogeneous information signals. Hedging instruments are represented by Arrow securities, which may be viewed as a proxies for more complicated financial contingent claims. Within each of the three model settings we have shown that, when agents adapt their behavior based upon reinforcement learning, a general mechanism for potential instability applies. In particular, the presence of more Arrow securities causes the primary bifurcation at the onset of instability to occur earlier. Moreover, 


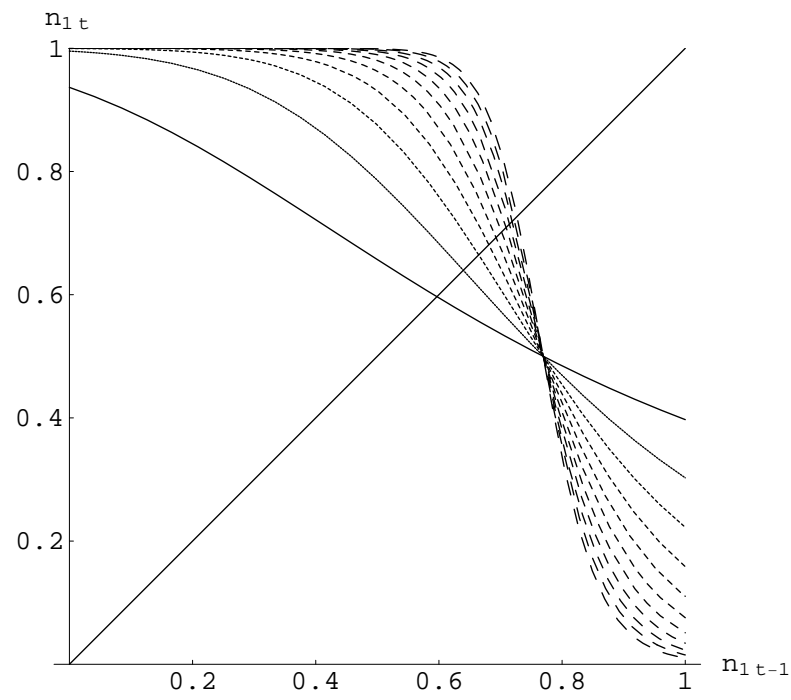

Figure 5: Information dynamics for two types, $\tau_{1}=0.35, \tau_{2}=0$. Parameter values are $\beta=10, a=1, C_{1}=0.5, C_{2}=0$. In the picture the number of information assets is increasing from 0 to 10 . The fixed point is losing stability in a period-doubling bifurcation as the number $n$ of information assets increases.

adding Arrow securities to the market may destabilize experience based market dynamics. We have provided explicit examples where adding Arrow securities triggers generic bifurcation routes (Hopf, saddle-node or period-doubling) to complex dynamics.

How general are these results? Clearly, they will not always hold. There may be other, stabilizing forces that offset the potential instability. For example, for analytic tractability we have have focussed on a two-period setting. An interesting question is what happens if we increase the time horizons of agents in the model and decrease the rates at which agents discount the future. This kind of force increases intertemporal smoothing which should lead to the result that risk sharing is not impeded by the incompleteness of markets. That in turn, we would conjecture, should mitigate any forces towards instability. See Levine and Zame (1996), (2001) for this kind of argument.

Furthermore one might think that increasing the horizon towards infinity and decreasing the discount rate towards zero might unleash forces that drive out any agent beliefs that are not fully structural rational expectations. Indeed the papers of Blume and Easley (2006) and Sandroni (2000) show exactly this type of result in an infinite horizon intertemporal general equilibrium complete markets setting. However, Beker and Chattopadhyay (2005) have recently shown that this result does not hold in certain incomplete markets settings. Blume and Easley (2006) also show that the market selection hypothesis may fail when markets are incomplete. 
Parenthetically we remark that the papers of Blume and Easley and Sandroni study belief heterogeneity and learning when there is agent disagreement about the probabilities of the states of the world that "drive" the formation of prices whereas we study agent disagreement and learning about the future prices themselves. It might be argued that the probabilities of the states of the world are "objective" in the sense that how well the markets function in pricing securities does not impact these state probabilities, so they might be "easier" to learn. Whereas asset prices themselves are impacted by interactions of traders within the markets. It seems plausible that in this "extra layer" of complexity, market agents could form beliefs about other market agents beliefs, and so on, much like Keynes's famous beauty contest metaphor. Such an expectational "hall of mirrors" should not play a role in the learning of the "objective" state probabilities themselves. We believe the study of differences in learning of "objective" state probabilities such as the probability of earnings at some future date being some specific amount versus learning the prices of assets that are claims on such future earnings is an important topic for future research. See Magill and Quinzii (1994) for pioneering work in this area, see Santos and Woodford (1997) for general techniques for dealing with bubbles and other issues that arise in infinite horizon general equilibrium models, and see Beker and Chattopadhyay (2005) and Sandroni (2005) for recent works on the impact of incomplete markets on forcing homogeneity of beliefs.

But this work on infinite horizon general equilibrium models with incomplete and complete markets does not treat learning systems. What would happen if this work were generalized to include reinforcement learning like that discussed in our current paper? We do not know and this is an important topic for future research. However, we would like to make an educated guess here. We think that a good guess would be this. Bifurcations towards instability would still tend to occur "earlier" when more risk hedging markets are added because this still would tend to allow (and encourage) agents to take larger positions whatever their predictors about the future may be. Hence under reinforcement learning this force will still lead to stronger reinforcement for relatively successful predictors. Thus we believe the potential for increased instability of reinforcement learning will still be present in more general markets. However, we also believe that increased horizons, lower discount rates, and more Arrow securities will cause increased homogenization of beliefs (i.e. increased concentration towards predictors that more closely approximate fully structural rational expectations predictors, even if these predictors are more costly, provided costs are sufficiently modest). Which force will "win"? The search for an answer in more elaborate models will be an exciting area for future research. 


\section{References}

[1] Amir, R., Evstigneev, I.V., Hens, T. and Schenk-Hoppé, K.R. (2005), Market selection and survival of investment strategies, Journal of Mathematical Economics 41, 105-122.

[2] Anderson, B. and Moore, J. (1979), Optimal filtering, Prentice-Hall: Englewood Cliffs, New Jersey.

[3] Anderson, S., de Palma, A. and Thisse, J., (1993), Discrete choice theory of product differentiation, MIT Press, Cambridge.

[4] Araújo, A. and Sandroni, A. (1999), On the convergence to homogeneous expectations when markets are complete, Econometrica 67, 663-672.

[5] Arifovic, J. (1994), Genetic algorithm learning and the cobweb model, Journal of Economic Dynamics and Control 18, 3-28.

[6] Arrowsmith, D.K. and Place, C.M. (1995), An introduction to dynamical systems, Cambridge University Press, Cambridge.

[7] Arthur, W.B. (1994), Complexity in economic theory. Inductive reasoning and bounded rationality, American Economic Review, AEA Papers and Proceedings, 84, 406-411.

[8] Arthur, W.B., Holland, J.H., LeBaron, B., Palmer, R. and Taylor, P. (1997) Asset pricing under endogenous expectations in an artificial stock market, in Arthur, W., Lane, D. and Durlauf, S., (eds) The economy as an evolving complex system II, Addison-Wesley.

[9] Barberis, N. and Thaler, R. (2003), A survey of behavioral finance, Handbook of the Economics of Finance, Edited by G.M. Constantinidis, M. Harris and R. Stulz, Elsevier.

[10] Beker, P. and Chattopadhyay, S. (2006), Economic survival when markets are incomplete, Working paper, University of Alicante.

[11] Benhabib, J. and Day, R.H. (1982), A characterization of erratic dynamics in the overlapping generations model, Journal of Economic Dynamics and Control 4, 37-55.

[12] Blume, L. and Easley, D. (1992), Evolution and market behavior, Journal of Economic Theory 58, 9-40.

[13] Blume, L. and Easley, D. (2006), If you're so smart, why aren't you rich? Belief selection in complete and incomplete markets, Econometrica 74, 929-1012.

[14] Boldrin, M. and Woodford, M. (1990), Equilibrium models displaying endogenous fluctuations and chaos: a survey, Journal of Monetary Economics 25, 189222.

[15] Branch, W.A. (2004), The theory of rationally heterogeneous expectations: evidence from survey data on inflation expectations, Economic Journal 114, 592621. 
[16] Branch, W.A. and Evans, G.W. (2006), Intrinsic heterogeneity in expectation formation, Journal of Economic Theory 127, 264-295.

[17] Brock, W.A. (1990), Overlapping generations models of money and transactions costs, In: Friedman, B., Hahn, F., (eds), Handbook of Monetary Economics, Volume 1, North Holland: Amsterdam, 263-295.

[18] Brock, W.A. and de Fontnouvelle, P. (2000), Expectational diversity in monetary economics, Journal of Economic Dynamics and Control 24, 725-759.

[19] Brock, W.A. and Hommes, C.H. (1997), A rational route to randomness, Econometrica 65, 1059-1095.

[20] Brock, W.A., and Hommes, C.H. (1998), Heterogeneous beliefs and routes to chaos in a simple asset pricing model, Journal of Economic Dynamics and Control 22, 1235-1274.

[21] Brock, W.A., Hommes, C.H. and Wagener, F.O.O. (2005), Evolutionary dynamics in markets with many trader types, Journal of Mathematical Economics 41, $7-42$.

[22] Bullard, J. (1994), Learning equilibria, Journal of Economic Theory 64, 468-485.

[23] Bullard, J., and Duffy, J. (1998), Learning and the stability of cycles, Macroeconomic Dynamics 2, 22-48.

[24] Bullard, J. and Duffy, J. (2001), Learning and excess volatility, Macroeconomic Dynamics 5, 272-302.

[25] Cass, D. (2006), Musings on the Cass trick, Journal of Mathematical Economics, forthcoming.

[26] Chiarella, C. and He, X. (2003), Heterogeneous beliefs, risk and learning in a simple asset pricing model with a market maker, Macroeconomic Dynamics 7 , 503-536.

[27] DeGrauwe, P. and Grimaldi, M. (2006), Exchange rate puzzles: A tale of switching attractors, European Economic Review 50, 1-33.

[28] DeLong, J.B., Shleifer, A., Summers, L.H. and Waldmann, R.J. (1990a), Noise trader risk in financial markets, Journal of Political Economy 98, 703-738.

[29] DeLong, J.B., Shleifer, A., Summers, L.H. and Waldmann, R.J. (1990b), Positive feedback investment strategies and destabilizing rational speculation, Journal of Finance 45, 379-395.

[30] Evans, G.W. and Honkapohja, S, (2001), Learning and expectations in macroeconomics, Princeton University Press, Princeton.

[31] Evstigneev, I.V., Hens, T. and Schenk-Hoppé, K.R. (2002), Market selection of financial trading strategies: global stability, Mathematical Finance 12, 329-339.

[32] de Fontnouvelle, P. (2000), Information dynamics in financial markets, Macroeconomic Dynamics 4, 139-169. 
[33] Gaunersdorfer, A. (2000), Endogenous fluctuations in a simple asset pricing model with heterogeneous beliefs, Journal of Economic Dynamics and Control 24, 799-831.

[34] Goldbaum, D. (2005), Market efficiency and learning in an endogenously unstable environment, Journal of Economic Dynamics and Control 29, 953-978.

[35] Goldbaum, D. (2006), Self-organization and the persistence of noise in financial markets, Journal of Economic Dynamics and Control 30, 1837-1855.

[36] Grandmont, J.-M. (1985), On endogenous competitive business cycles, Econometrica 53, 995-1046.

[37] Grandmont, J.-M. (1986), Nonlinear economic dynamics, Special issue of the Journal of Economic Theory 40, 1-195.

[38] Grandmont, J.-M. (1988), Nonlinear difference equations bifurcations and chaos: an introduction, CEPREMAP, June 1988.

[39] Grandmont, J.-M. (1998), Expectation formation and stability in large socioeconomic systems, Econometrica 66, 741-781.

[40] Grandmont, J.-M. and Younes, Y. (1972), On the role of money and the existence of a monetary equilibrium, Review of Economic Studies 39, 355-372.

[41] Grossman, S.J. and Stiglitz, J.E. (1980), On the impossibility of informationally efficient markets, American Economic Review 70, 393-408.

[42] Guckenheimer, J. and Holmes, P. (1983), Nonlinear oscillations, dynamical systems, and bifurcations of vector fields, Springer Verlag, New York.

[43] Guesnerie, R. (2002), Anchoring economic predictions in common knowledge, Econometrica 70, 439-480.

[44] Hellwig, M. (1980), On the aggregation of information in competitive markets, Journal of Economic Theory 22, 477-489.

[45] Hens, T. and Schenk-Hoppé, K.R. (2005), Evolutionary stability of portfolio rules in incomplete markets, Journal of Mathematical Economics 41, 43-66.

[46] Hommes, C.H. (2006), Heterogeneous agent models in economics and finance, In: Tesfatsion, L. and Judd, K.L., (eds), Handbook of Computational Economics, Volume 2: Agent-Based Computational Economics, chapter 23, North-Holland, Amsterdam, 1109-1186.

[47] Kirman, A. (1993), Ants, rationality and recruitment, Quarterly Journal of Economics $108,137-156$.

[48] Kirman, A. (2006), Heterogeneity in economics, Journal of Economic Interaction and Coordination 1, 89-117.

[49] Kurz, M. (1994), Endogenous economic fluctuations: studies in the theory of rational beliefs, Springer Verlag, Berlin. 
[50] Kuznetsov, Y. (1995), Elements of applied bifurcation theory, Springer Verlag, New York.

[51] LeBaron, B. (2006), Agent-based Computational Finance, Handbook of Computational Economics, Volume 2: Agent-Based Computational Economics, chapter 24, Edited by L. Tesfatsion and K.L. Judd, North-Holland, Amsterdam, 11871233.

[52] LeBaron, B., Arthur, W.B. and Palmer, R. (1999), Time series properties of an artificial stock market, Journal of Economic Dynamics and Control 23, 14871516.

[53] Levine, D. and Zame, W. (1996), Debt Constraints and Equilibrium in Infinite Horizon Economies with Incomplete Markets, Journal of Mathematical Economics 26, 103-131.

[54] Levine, D. and Zame, W. (2001), Does market incompleteness matter?, Econometrica 70, 1805-1840.

[55] Medio, A. and Lines, M. (2001) Non-linear dynamics. A primer, Cambridge University Press.

[56] Magill, M. and Quinzii, M. (1994), Infinite horizon incomplete markets, Econometrica 62, 853-880.

[57] Marcet, A., and Nicolini, J.P. (2003), Recurrent hyperinflations and learning, American Economic Review 93, 1476-1498.

[58] Marcet, A., and Sargent, T.J. (1989), Convergence of least squares learning in mechanisms in self referential linear stochastic models, Journal of Economic Theory 48, 337-368.

[59] Marimon, R. (1997), Learning from learning in economics, In: Kreps, D. and Wallis, K. (eds), Advances in Economics and Econometrics: Theory and Applications, Volume I, Cambridge University Press, 278-315

[60] Rajan, G.R. (2005), Has financial development made the world riskier? NBER Working Paper Series, No. 11728, November 2005.

[61] Sandroni, A. (2000), Do markets favor agents able to make accurate predictions? Econometrica 68, 1303-1341.

[62] Sandroni, A. (2005), Market selection when markets are incomplete, Journal of Mathematical Economics 41, 91-104.

[63] Santos, M. and Woodford, M. (1997), Rational asset pricing bubbles, Econometrica $65,19-57$.

[64] Sargent, T.J. (1993): Bounded rationality in macroeconomics, Clarendon Press, Oxford.

[65] Scheinkman, J.A. and Xiong, W. (2004), Heterogeneous beliefs, speculation and trading in financial markets, In: Carmona, R.A. et al. (eds), Paris-Princeton 
Lectures in Mathematical Finance 2003, Lecture Notes in Mathematics 1847, Springer Verlag, Berlin, 217-250.

[66] Schönhofer, M. (1999), Chaotic learning equilibria, Journal of Economic Theory 89, 1-20.

[67] Sethi, R. and Franke, R. (1995), Behavioural heterogeneity under evolutionary pressure: macroeconomic implications of costly optimization, Economic Journal $105,583-600$.

[68] Shiller, R. J. (2003), The new financial order: risk in the 21st century, Princeton University Press.

[69] Skogestad, S. and Postlethwaite, I. (1996), Multivariable feedback control, John Wiley and Sons, New York.

[70] Woodford, M. (1990): Learning to believe in sunspots, Econometrica 58, 277307. 


\section{A Proof of the lemma}

Here the proof of the matrix lemma 1 is given.

LEMMA 3. Let $Q_{n+1}$ be a symmetric matrix of the form $\left(\begin{array}{cc}Q_{n} & r \\ r^{T} & s\end{array}\right)$, and let $\tilde{w}=$ $\left(w, w_{0}\right)$. Then

$$
\left\langle\tilde{w}, Q_{n+1}^{-1} \tilde{w}\right\rangle=\left\langle w, Q_{n}^{-1} w\right\rangle+\frac{\left(w_{0}-\left\langle r, Q_{n}^{-1} w\right\rangle\right)^{2}}{s-\left\langle r, Q_{n}^{-1} r\right\rangle} .
$$

Moreover,

$$
\operatorname{det} Q_{n+1}=\operatorname{det} Q_{n}\left(s-\left\langle r, Q_{n}^{-1} r\right\rangle\right) .
$$

\section{Proof}

Set $\tilde{\xi}=\left(\xi, \xi_{0}\right)$, and let $\tilde{w}=Q_{n+1} \tilde{\xi}$. First $\tilde{\xi}$ will be determined, and then the quadratic form $\left\langle\tilde{w}, Q_{n+1}^{-1} \tilde{w}\right\rangle$ is evaluated by computing $\langle\tilde{w}, \tilde{\xi}\rangle$.

In components, $\tilde{w}=Q_{n+1} \tilde{\xi}$ reads as

$$
\begin{gathered}
w=Q_{n} \xi+\xi_{0} r, \\
w_{0}=\langle r, \xi\rangle+\xi_{0} s .
\end{gathered}
$$

Solving $\xi$ in terms of $w$ and $\xi_{0}$ yields

$$
\xi=Q_{n}^{-1} w-\xi_{0} Q_{n}^{-1} r
$$

Substitution into the second equation yields a relation for $\xi_{0}$ :

$$
\xi_{0}=\frac{w_{0}-\left\langle r, Q_{n}^{-1} w\right\rangle}{s-\left\langle r, Q_{n}^{-1} r\right\rangle} .
$$

From this, the vector $\xi$ is obtained as

$$
\xi=Q_{n}^{-1} w+\frac{\left\langle r, Q_{n}^{-1} w\right\rangle-w_{0}}{s-\left\langle r, Q_{n}^{-1} r\right\rangle} Q_{n}^{-1} r .
$$

Finally,

$$
\begin{aligned}
\left\langle\tilde{w}, Q_{n+1}^{-1} \tilde{w}\right\rangle & =\langle\tilde{w}, \tilde{\xi}\rangle=\langle w, \xi\rangle+w_{0} \xi_{0} \\
& =\left\langle w, Q_{n}^{-1} w\right\rangle+\frac{\left\langle r, Q_{n}^{-1} w\right\rangle-w_{0}}{s-\left\langle r, Q_{n}^{-1} r\right\rangle}\left\langle w, Q_{n}^{-1} r\right\rangle+\frac{w_{0}^{2}-\left\langle r, Q_{n}^{-1} w\right\rangle w_{0}}{s-\left\langle r, Q_{n}^{-1} r\right\rangle} \\
& =\left\langle w, Q_{n}^{-1} w\right\rangle+\frac{\left(w_{0}-\left\langle r, Q_{n}^{-1} w\right\rangle\right)^{2}}{s-\left\langle r, Q_{n}^{-1} r\right\rangle} .
\end{aligned}
$$


This proves the first statement of the lemma. For the second statement, let $C$ be the orthogonal matrix such that $Q_{n}=C^{T} \Lambda C$, with $\Lambda$ a diagonal matrix, let $\tilde{C}=$ $\left(\begin{array}{cc}C & 0 \\ 0 & 1\end{array}\right)$ and, finally, let $v=C^{T} r$. Then

$$
\begin{aligned}
\operatorname{det} Q_{n+1} & =\operatorname{det} \tilde{C}^{T} Q_{n+1} \tilde{C}=\operatorname{det}\left(\begin{array}{cc}
C^{T} & 0 \\
0 & 1
\end{array}\right)\left(\begin{array}{cc}
Q_{n} & r \\
r^{T} & s
\end{array}\right)\left(\begin{array}{cc}
C & 0 \\
0 & 1
\end{array}\right) \\
& =\operatorname{det}\left(\begin{array}{cc}
\Lambda & v \\
v^{T} & s
\end{array}\right)=\operatorname{det} \Lambda\left(s-\sum_{i} \frac{v_{i}^{2}}{\lambda_{i}}\right)=\operatorname{det} \Lambda\left(s-\left\langle v, \Lambda^{-1} v\right\rangle\right) \\
& =\operatorname{det} Q_{n}\left(s-\left\langle r, Q_{n}^{-1} r\right\rangle\right) .
\end{aligned}
$$

\section{B Proof of bifurcations in 2-type example}

In this appendix we investigate local stability and bifurcation of steady states in the 2type example with (near-)fundamentalists versus trend followers, with fitness given by risk-adjusted profits, as discussed in section 2 . To investigate the stability of the steady states, we linearize the dynamics at a steady state equilibrium $x_{*}$ by writing $x_{t}=$ $x_{*}+y_{t}$, leading to

$$
\begin{aligned}
R x_{*}+R y_{t}=n_{1 *} & +n_{2 *} x_{*} \\
& +\left(n_{1 t}-n_{1 *}\right)+\left(n_{2 t}-n_{2 *}\right) x_{*}+n_{2 t}\left(y_{t-1}+g\left(y_{t-1}-y_{t-2}\right)\right) .
\end{aligned}
$$

This simplifies to

$$
R y_{t}=\left(n_{1 t}-n_{1 *}\right)\left(1-x_{*}\right)+\left(1-n_{1 t}\right)\left(y_{t-1}+g\left(y_{t-1}-y_{t-2}\right)\right) .
$$

Note that $u_{2 *}=0$ and $u_{2 t}=-\left(y_{t-1}-y_{t-3}-g\left(y_{t-3}-y_{t-4}\right)\right)^{2}$. We compute

$$
\begin{aligned}
n_{1 t}-n_{1 *} & =\frac{1}{1+\mathrm{e}^{-\beta\left(u_{1 t}-u_{2 t}\right)}}-\frac{1}{1+\mathrm{e}^{-\beta\left(u_{1 *}-u_{2 *}\right)}} \\
& =-\frac{2 \beta\left(x_{*}-1\right) \mathrm{e}^{\beta\left(x_{*}-1\right)^{2}}}{\left(1+\mathrm{e}^{\beta\left(x_{*}-1\right)^{2}}\right)^{2}} y_{t-1}+\mathrm{O}\left(\left\|\left(y_{t-1}, \cdots, y_{t-4}\right)\right\|^{2}\right) .
\end{aligned}
$$

This yields the linearized dynamics

$$
\begin{aligned}
R y_{t}=\frac{2 \beta\left(x_{*}-1\right)^{2} \mathrm{e}^{\beta\left(x_{*}-1\right)^{2}}}{\left(1+\mathrm{e}^{\beta\left(x_{*}-1\right)^{2}}\right)^{2}} y_{t-1}+\left(1-n_{1 *}\right)\left(y_{t-1}+g\left(y_{t-1}-y_{t-2}\right)\right) \\
\quad+\mathrm{O}\left(\left\|\left(y_{t-1}, \cdots, y_{t-4}\right)\right\|^{2}\right) .
\end{aligned}
$$


Bifurcations. Introduce

$$
c_{1}=c_{1}(\beta, \varepsilon)=\frac{1}{R} \frac{2 \beta\left(x_{*}-1\right)^{2} \mathrm{e}^{\beta\left(x_{*}-1\right)^{2}}}{\left(1+\mathrm{e}^{\beta\left(x_{*}-1\right)^{2}}\right)^{2}}, \quad c_{2}=\frac{1}{R}\left(1-n_{1 *}\right) .
$$

Then the characteristic equation of the linearized dynamics is

$$
\sigma^{2}=c_{1} \sigma+c_{2}((1+g) \sigma-g)
$$

or

$$
\sigma^{2}-\left(c_{1}+(1+g) c_{2}\right) \sigma+g c_{2}=0 \text {. }
$$

Its roots $\sigma_{1,2}$ satisfy

$$
\sigma_{1}+\sigma_{2}=c_{1}+(1+g) c_{2}, \quad \sigma_{1} \sigma_{2}=g c_{2} .
$$

Necessary for a saddle-node bifurcation is that $\sigma_{1}=1$. This implies

$$
g c_{2}=\sigma_{2}=\left(\sigma_{1}+\sigma_{2}\right)-\sigma_{1}=c_{1}+(1+g) c_{2}-1,
$$

yielding the condition

$$
c_{1}+c_{2}=1
$$

Likewise, a period-doubling bifurcation may be obtained only if $\sigma_{1}=-1$, yielding

$$
g c_{2}=-\sigma_{2}=-\left(\sigma_{1}+\sigma_{2}\right)+\sigma_{1}=-c_{1}-(1+g) c_{2}-1 \text {, }
$$

yielding

$$
c_{1}+(1+2 g) c_{2}=-1
$$

Since $c_{1}, c_{2}>0$, this possibility is excluded.

Finally, the necessary condition for a Hopf bifurcation is that $\sigma_{1}=\overline{\sigma_{2}}$ and $\left|\sigma_{1}\right|=1$, but $\sigma_{1} \neq \pm 1$, leading to

$$
g c_{2}=1 \quad \text { and } \quad\left|c_{1}+(1+g) c_{2}\right|<2 .
$$

This can be simplified to

$$
g c_{2}=1 \quad \text { and } \quad-3<c_{1}+c_{2}<1
$$


Asymptotics. We return to the steady state equation (29). If we solve this equation for the exponent $u=\beta\left(x_{*}-1\right)^{2}$ of the exponential, we obtain

$$
u=\log \left(\frac{1}{R-1} \frac{1}{x_{*}}-\frac{R}{R-1}\right) .
$$

We see that $u$ is strictly decreasing in $x_{*}$. Note for instance that the locus of equilibria given in figure 2 can be described as a regular curve parametrized by $u$.

Moreover, in terms of $u$, the expressions for $c_{1}$ and $c_{2}$ read as

$$
c_{1}=\frac{2}{R} \frac{u \mathrm{e}^{u}}{\left(1+\mathrm{e}^{u}\right)^{2}}, \quad c_{2}=\frac{1}{R} \frac{\mathrm{e}^{u}}{1+\mathrm{e}^{u}} .
$$

If $\beta=0$, then $u=0$ and $c_{1}=0$ and $c_{2}=1 /(2 R)$. Since $0<x_{*}<1 / R$, we have that $\left(x_{*}-1\right)^{2}>(1-1 / R)^{2}$ uniformly in $\beta$. Therefore, if $\beta \rightarrow \infty$, then $u \rightarrow \infty$ and

$$
n_{1} \rightarrow 0, \quad c_{1} \rightarrow 0, \quad c_{2} \rightarrow \frac{1}{R}
$$

Saddle-node bifurcation. The saddle-node condition $c_{1}+c_{2}=1$ reads

$$
\frac{2}{R} \frac{u \mathrm{e}^{u}}{\left(1+\mathrm{e}^{u}\right)^{2}}+\frac{1}{R} \frac{\mathrm{e}^{u}}{1+\mathrm{e}^{u}}=1
$$

and after some re-writing

$$
u=\frac{1}{2}\left(1+\mathrm{e}^{u}\right)\left(R \mathrm{e}^{-u}+R-1\right) .
$$

Denoting the right hand side by $\rho(u)$, we compute the derivative

$$
\rho^{\prime \prime}(u)=\frac{1}{2}(R-1) \mathrm{e}^{u}+\frac{1}{2} R \mathrm{e}^{-u}
$$

and we see that $\rho^{\prime \prime}>0$ for all $u$, and hence that $\rho$ is convex. Since the number of intersections of a line with the graph of a convex function is at most two, we conclude from equation (90) that we have at most two saddle-node bifurcation points. Moreover, we have a cusp bifurcation point if the two saddle-node points coincide, which is equivalent to the condition $\rho^{\prime}(u)=1$. This condition reads as

$$
(R-1) \mathrm{e}^{u}-R \mathrm{e}^{-u}-2=0 .
$$

Rearranging and completing the square yields

$$
\begin{array}{r}
\mathrm{e}^{2 u}-\frac{2}{R-1} \mathrm{e}^{u}-\frac{R}{R-1}=0, \\
\left(\mathrm{e}^{u}-\frac{1}{R-1}\right)^{2}=\frac{1+R(R-1)}{(R-1)^{2}}, \\
\mathrm{e}^{u}=\frac{1+\sqrt{1+R(R-1)}}{R-1},
\end{array}
$$

since the other root is always negative. We see that we have a unique cusp bifurcation in the system. 
Hopf bifurcation. The Hopf bifurcation condition $g c_{2}=1$ reads

$$
1=g c_{2}=\frac{g}{R}\left(1-\frac{1}{1+\mathrm{e}^{u}}\right)
$$

yielding

$$
u=\log \frac{R}{g-R} .
$$

Substitution in equation (89) yields that

$$
\frac{R}{g-R}=\frac{1}{R-1} \frac{1}{x_{*}}-\frac{R}{R-1}
$$

resulting in

$$
x_{*}=\frac{g-R}{R(g-1)} .
$$

Substituting this back into $u=\beta\left(x_{*}-1\right)^{2}$ and using relation (91) yields

$$
\begin{aligned}
\beta_{\text {Hopf }} & =\left(\frac{g-R}{R(g-1)}-1\right)^{-2} \log \frac{R}{g-R} \\
& =\frac{R^{2}}{g^{2}} \frac{(g-1)^{2}}{(R-1)^{2}} \log \frac{R}{g-R} .
\end{aligned}
$$

Note that we still have to verify the Hopf inequality condition that the eigenvalues are complex. For this we compute $c_{1}+c_{2}$, making use of (91) and of $c_{2}=1 / g$. This yields

$$
c_{1}+c_{2}=\frac{1}{g}+2 \frac{g-R}{g^{2}} \log \frac{R}{g-R}=\frac{1}{g}\left(1+2 \frac{g-R}{g} \log \frac{R}{g-R}\right) .
$$

Recalling that $c_{1}, c_{2}>0$, this yields the inequality condition

$$
\frac{g-R}{g} \log \frac{R}{g-R}<\frac{g-1}{2}
$$

or

$$
-\frac{g-R}{R} \log \frac{g-R}{R}<\frac{g(g-1)}{2 R}
$$

Note that since $\lim _{x \downarrow 0} x \log x=0$, this condition is satisfied if $0<g-R \ll 1$, that is, when $g>R$ but $g$ sufficiently close to $R$. 


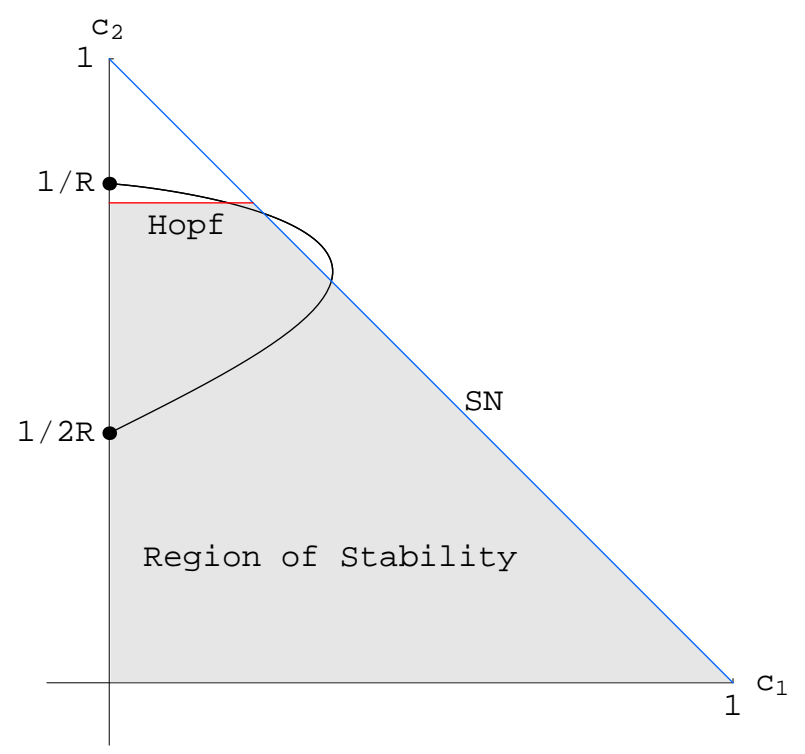

Figure 6: Bifurcation diagram in terms of the coefficients $c_{1}$ and $c_{2}$. The curve is plotted for values of $u=\beta\left(x_{*}-1\right)^{2}$ between $u=0$, corresponding to the point $\left(c_{1}, c_{2}\right)=$ $(0,1 /(2 R))$, and $u=\infty$, corresponding to $\left(c_{1}, c_{2}\right)=(0,1 / R)$. Note that $x_{*}$ decreases monotonically along the curve.

\section{Proof of the existence of an equilibrium price}

In this section, the existence of an equilibrium price in the two periods overlapping generations model will be shown.

C.1 Formulation of the problem. The model is populated by many agents. Each agent lives for two periods. In period $i$, he obtains an endowment $w_{i}$ of some consumption good that is non-storable. We assume that these endowments are the same for all agents.

In the first period it is common knowledge that in the second period, the world can be in $S$ different states; state $j$ occurs with probability $\alpha_{j}$. The agents can trade a risky asset and $n$ Arrow securities. The $j$ 'th Arrow security is bought at a price $p^{j}$; if in the next period, the state of the world is equal to $j$, this Arrow security pays a dividend 1 , otherwise 0 , before perishing. The risky asset is bought at a price $p^{0}$ and yields a dividend $y^{s}$ in state of the world $s$.

There are $H$ different types of agents; an agent of type $h$ is characterized by the fact that he expects to sell the risky asset in the second period at a price $p_{h t+1}^{0}$. For a given price vector $\left(p^{0}, p^{1}, \cdots, p^{n}\right)$, trader type $h$ determines his demand $z_{h}(p)=$ $\left(z_{h}^{-1}(p), z_{h}^{0}(p), \cdots, z_{h}^{n}(p)\right)$; here $z_{h}^{-1}$ is his demand of the consumption good in the first period; $\zeta^{0}+z_{h}^{0}$ his demand of the risky asset (so in what follows $z_{h}^{0}$ represents 
excess demand for the risky asset) and $z_{h}^{j}$, for $j=1, \cdots, n$, his demand of the $j$ 'th Arrow security. The trader determines these demands by maximizing his expected utility

$$
U_{h}\left(z_{h}^{-1}, z_{h}^{0}, z_{h} ; p\right)=u_{1}\left(c_{1 h}\right)+\sum_{s=1}^{S} u_{2}\left(c_{2 h}^{s}\right)
$$

subject to the restrictions

$$
\begin{aligned}
0 & \geq z_{h}^{-1}+\sum_{j=0}^{n+1} p^{j} z_{h}^{j}, \\
c_{1 h} & =w_{1}+z_{h}^{0} \geq 0, \\
c_{2 h}^{s} & =w_{2}+\left(p_{h t+1}^{0}+y^{s}\right)\left(\zeta^{0}+z_{h}^{0}\right)+z_{h}^{s} \geq 0, \quad s=1, \cdots, n, \\
c_{2 h}^{s} & =w_{2}+\left(p_{h t+1}^{0}+y^{s}\right)\left(\zeta^{0}+z_{h}^{0}\right) \geq 0, \quad s=n+1, \cdots, S .
\end{aligned}
$$

In this setup, equilibrium is defined as a price vector $p$ for which the aggregate excess demand of all assets vanishes:

$$
\sum_{h=1}^{H} n_{h t} z_{h}^{j}(p)=0, \quad j=-1,0, \cdots, n .
$$

It is assumed that $u_{1}$ and $u_{2}$ are strictly concave, and that they moreover satisfy the following Inada conditions:

$$
\lim _{c \downarrow 0} u_{j}^{\prime}(c)=\infty, \quad \text { and } \quad \lim _{c \rightarrow \infty} u_{j}^{\prime}(c)=0, \quad j=1,2 .
$$

\section{C.2 Reformulation of the problem.}

Reducing the number of states of the world. To treat the individual optimization problem, it is convenient to combine the utilities in the states $s=n+1, \cdots, S$, for which there are no Arrow securities available, into a new utility function. Define for this $y_{*}=\min _{n+1 \leq s \leq S} y^{s}$, set $\bar{\alpha}_{j}=\alpha_{j}$ for $j=1, \cdots, n$ and $\bar{\alpha}_{n+1}=\sum_{s=n+1}^{S} \alpha_{s}$ and introduce

$$
\bar{u}_{2}\left(w_{2}^{n+1}+\left(p_{h t+1}^{0}+y_{*}\right) z_{h}^{0}\right) \bar{\alpha}_{n+1}=\sum_{s=n+1}^{S} u_{2}\left(w_{2}+\left(p_{h t+1}^{0}+y^{s}\right)\left(\zeta^{0}+z_{h}^{0}\right)\right) \alpha_{s} .
$$

Note that $\bar{u}_{2}$ is strictly concave, and that $\lim _{c \downarrow 0} \bar{u}_{2}^{\prime}(c)=\infty$ and $\lim _{c \rightarrow \infty} \bar{u}_{2}^{\prime}(c)=0$. In this way, we reduce the possible states of the world to $n+1$. Introduce also

$$
q_{h}^{j}=p_{h t+1}^{n+1}+y^{j}, \quad j=1, \cdots, n, \quad q_{h}^{n+1}=p_{h t+1}^{n+1}+y_{*} .
$$


Normalizing prices. As a second transformation, introduce new prices $\hat{p}$ by setting

$$
\hat{p}^{0}=\frac{1}{\sum_{k=1}^{n+1} p^{k}}, \quad p^{j}=\frac{\hat{p}^{j}}{\hat{p}^{0}}, \quad \text { for } \quad j=1, \cdots, n+1 .
$$

Note that $\sum_{j=1}^{n+1} \hat{p}^{j}=1$.

Each agent faces now the transformed problem of maximizing

$$
\begin{aligned}
U_{h} & =u_{1}\left(w_{1}+z^{-1}\right) \\
& +\sum_{j=1}^{n} u_{2}\left(w_{2}+q_{h}^{j}\left(\zeta^{0}+z^{0}\right)+z^{j}\right) \bar{\alpha}_{j}+\bar{u}_{2}\left(w_{2}+q_{h}^{0}\left(\zeta^{0}+z^{0}\right)\right) \bar{\alpha}_{n+1},
\end{aligned}
$$

subject to the budget restriction $\sum_{k=-1}^{n} \hat{p}^{k} z^{k} \leq 0$.

The price $\hat{p}^{-1}$ is interpreted as the price of the consumption good in terms of which the trades in the first period are performed. As an effect of these transformations, prices are now restricted to the (compact) unit simplex

$$
\Delta=\left\{\hat{p} \in \mathbb{R}^{n+2} \mid \sum_{k=0}^{n+1} \hat{p}^{k}=1, \hat{p}^{j} \geq 0, j=0, \cdots, n+1\right\} .
$$

In the following, we shall drop the hats on the prices.

Short selling restrictions. The traders face the following short selling restriction on the risky asset

$$
z_{h}^{0} \geq-\frac{w_{2}}{q_{h}^{0}}=m_{h}^{0}
$$

If the total supply of the risky asset is denoted by $\zeta^{0}$, we have

$$
\sum_{h=1}^{H} n_{h}^{0} z_{h}^{0}=\zeta^{0}
$$

Combining this equality with the short selling constraints, it follows that

$$
z_{h}^{0} \leq \zeta^{0}+\sum_{\substack{k=1 \\ k \neq h}}^{H} \frac{n_{k}^{0}}{n_{h}^{0}} \frac{w_{2}}{q_{k}^{0}}=M_{h}^{0}
$$

Using this inequality, we can also bound the demand for the Arrow securities from below

$$
z_{h}^{j} \geq-q_{h}^{j} z_{h}^{0}-w_{2} \geq-q_{h}^{j} M_{h}^{0}-w_{2}=m_{h}^{j} .
$$

Introduce also the constants

$$
m=\min _{h} \min _{j} m_{h}^{j} \quad \text { and } \quad M=\max _{h} \max _{j} M_{h}^{j} .
$$


Normalizing assets. Using the assets we have, we construct a new, second, set of assets, such that the $j$ 'th new asset pays nothing in any state of the world except the $j$ 'th. Roughly speaking, the risky asset is used to construct an additional Arrow security that pays nothing in the first $n$ states of the world and 1 in state $n+1$. It is important to note that this new set of assets is different for different types of agents.

Taking now the new $(n+1)$ Arrow securities as basic assets, we look for the corresponding demands $\tilde{z}$ and prices $\tilde{p}$ such that the two forms of the system are equivalent. For this, we obviously need to have that

$$
\tilde{z}^{j}=q_{h}^{j} z^{0}+z^{j}, \quad j=1, \cdots, n, \quad \tilde{z}^{0}=q_{h}^{0} z^{0} .
$$

Denote the matrix of this transformation by $A_{h}$, that is: $\tilde{z}=A_{h} z$. Its inverse is given by the equations

$$
z^{j}=\tilde{z}^{j}-\frac{q_{h}^{j}}{q_{h}^{0}} \tilde{z}^{0}, \quad j=1, \cdots, n, \quad z^{0}=\frac{1}{q_{h}^{0}} \tilde{z}^{0} .
$$

It follows that the prices of the new securities read as

$$
\tilde{p}^{j}=p^{j}, \quad j=1, \cdots, n, \quad \tilde{p}^{0}=\frac{1}{q_{h}^{0}} p^{0}-\sum_{k=1}^{n} \frac{q_{h}^{k}}{q_{h}^{0}} p^{k} .
$$

Note that we have the following bounds on the transformed demands (recall that $m<$ $0<M$ and that $m<z_{h}^{j}<M$ for all $j$ and $M$ ):

$$
\begin{aligned}
& \tilde{z}^{j} \leq q_{h}^{j} M+M \leq M \max _{j} \max _{h}\left(q_{h}^{j}+1\right)=\tilde{M}, \\
& \tilde{z}^{j} \geq q_{h}^{j} m+m \geq m \max _{j} \max _{h}\left(q_{h}^{j}+1\right)=\tilde{m} .
\end{aligned}
$$

Finally, we also introduce

$$
\begin{aligned}
v_{0}\left(\omega^{0}+\tilde{z}^{0}\right) & =u_{1}\left(w_{1}+z^{-1}\right) \\
v_{1}\left(\omega^{1}+\tilde{z}^{1}\right) & =\bar{u}_{2}\left(w_{2}+q_{h}^{0}\left(\zeta^{0}+z^{0}\right)\right) \alpha_{0}, \\
v_{j+1}\left(\omega^{j+1}+\tilde{z}^{j+1}\right) & =u_{2}\left(w_{2}+q_{h}^{j}\left(\zeta^{0}+z^{0}\right)+z^{j}\right) \alpha_{j}, \quad j=1, \cdots, n .
\end{aligned}
$$

Note that we defined the $\omega^{k}$ implicitly through these equations as

$$
\omega_{h}^{0}=w_{1}, \quad \omega_{h}^{1}=w_{2}+q_{h}^{0} \zeta^{0}, \quad \omega_{h}^{j}=w_{2}+q_{h}^{j} \zeta^{0}, \quad j=1, \cdots, n .
$$

C.3 Nonvanishing demands. We shall show that for a certain region close to the boundary of the price simplex $\Delta$ of the original prices, aggregate demand of at least one of the agents for at least one of the assets grows beyond all bounds. Since total supply of any asset is bounded, aggregate demand cannot vanish for such a price. 
In the following we shall drop the index $h$ on all quantities. The demand $\tilde{z}$ of type $h$ is given by the unique solution to the following maximization problem

$$
\operatorname{maximize} \tilde{U}(\tilde{z})=\sum_{k=0}^{n+1} v_{k}\left(\omega^{k}+\tilde{z}^{k}\right) \quad \text { restricted to } \quad \tilde{b}(\tilde{z})=\sum_{k=0}^{n+1} \tilde{p}^{k} \tilde{z}^{k}=0 .
$$

It necessarily is determined by the following Lagrange equations

$$
\nabla \tilde{U}=\lambda \nabla \tilde{b}, \quad \tilde{b}=0 .
$$

The first equation reads as

$$
v_{k}^{\prime}\left(\omega^{k}+\tilde{z}^{k}\right)=\lambda \tilde{p}^{k}, \quad k=0, \cdots, n+1
$$

First induction step. To show that demand of at least one asset is unlimited in a region close to the boundary of the asset, we proceed inductively. The first induction step is discussed at length. For a given $0<\varepsilon_{1} \ll 1$, consider the region $C_{j}$ of the price simplex for which

$$
0<\tilde{p}^{k}<\varepsilon_{1} \quad \text { if } \quad k \neq j .
$$

We consider two cases: $\tilde{p}^{j} \tilde{z}^{j} \leq-\delta$ and $\tilde{p}^{j} \tilde{z}^{j}>-\delta$.

In the first case

$$
\delta \leq-\tilde{p}^{j} \tilde{z}^{j}=\sum_{\substack{k=0 \\ k \neq j}}^{n+1} \tilde{p}^{k} \tilde{z}^{k} \leq \varepsilon_{1} \sum_{\substack{k=0 \\ k \neq j}}^{n+1} \tilde{z}^{k}
$$

which implies that

$$
\frac{\delta}{\varepsilon_{1}} \leq \tilde{z}^{k}
$$

for at least one $k \neq j$. Choosing

$$
0<\varepsilon_{1}<\frac{\delta}{\tilde{M}}
$$

where $\tilde{M}$ is as defined in (94) ensures that for $p \in C_{j}$ aggregate demand is non-zero. In the second case $\tilde{p}^{j} \tilde{z}^{j}>-\delta$, we have that

$$
\lambda \tilde{p}^{j}=v_{j}^{\prime}\left(\omega^{j}+\tilde{z}^{j}\right)<v_{j}^{\prime}\left(\omega^{j}-\frac{\delta}{\tilde{p}^{j}}\right)<v_{j}^{\prime}\left(\omega^{j}-\frac{\delta}{1-\varepsilon_{1}}\right),
$$

which implies that

$$
\lambda<\frac{v_{j}^{\prime}\left(\omega^{j}-\frac{\delta}{1-\varepsilon_{1}}\right)}{1-\varepsilon_{1}} \leq \max _{j} \frac{v_{j}^{\prime}\left(\omega^{j}-\frac{\delta}{1-\varepsilon_{1}}\right)}{1-\varepsilon_{1}} \stackrel{\text { def }}{=} K_{1} .
$$

From equation (96) we obtain then for $k \neq j$ that

$$
K_{1} \varepsilon_{1}>\lambda \tilde{p}^{k}=v_{k}^{\prime}\left(\omega^{k}+\tilde{z}^{k}\right) .
$$

As $v_{k}$ satisfies the Inada conditions, we find that there is a value $\kappa_{1}>0$ such that if $0<\varepsilon_{1}<\kappa_{1}$, then $\tilde{z}^{k}>\tilde{M}$. 
General induction step. The proof of the general $(i$ 'th) induction step runs along the same lines. Let $N=\{0, \cdots, n+1\}$. For $2 \leq i \leq n+1$, we consider a set $J=$ $\left\{j_{1}, \cdots, j_{i}\right\} \subset N$ of $i$ indices, as well as its complement $J^{c}=N \backslash J$. Both sets are necessarily non-empty.

Consider the region $C_{J}$ of the price simplex that satisfies

$$
0<\sum_{j \in J} \tilde{p}^{j}<\varepsilon_{i} \quad \text { if } \quad j \in J^{c}
$$

and, for any set $J_{-}$of $i-1$ indices

$$
\varepsilon_{i-1} \leq \sum_{j \in J_{-}} p^{j} \quad \text { if } \quad j \in J_{-}
$$

As before, we investigate two cases: $\sum_{j \in J} \tilde{p}^{j} \tilde{z}^{j} \leq-\delta$ and $\sum_{j \in J} \tilde{p}^{j} \tilde{z}^{j}>-\delta$.

In the first case

$$
\delta \leq-\sum_{j \in J} \tilde{p}^{j} \tilde{z}^{j}=\sum_{j \in J^{c}} \tilde{p}^{j} \tilde{z}^{j} \leq \varepsilon_{i} \sum_{j \in J^{c}} \tilde{z}^{j}
$$

which implies that

$$
\frac{\delta}{\varepsilon_{i}} \leq \tilde{z}^{j}
$$

for at least one $j \in J^{c}$. Choosing

$$
0<\varepsilon_{i}<\min \left\{\varepsilon_{i-1}, \frac{\delta}{\tilde{M}}\right\}
$$

with $\tilde{M}$ as in (94) ensures that for $p \in C_{J}$ aggregate demand is non-zero in this case.

In the second case $\sum_{j \in J} \tilde{p}^{j} \tilde{z}^{j}>-\delta$, we re-write this condition as

$$
0<\sum_{j \in J} \tilde{p}^{j}\left(\tilde{z}^{j}+\frac{\delta}{\sum_{j \in J} \tilde{p}^{j}}\right) .
$$

It follows then that for at least one index $k \in J$ we have

$$
0<\tilde{z}^{k}+\frac{\delta}{\sum_{j \in J} \tilde{p}^{j}}<\tilde{z}^{k}+\frac{\delta}{1-\varepsilon_{i}} .
$$

This leads to the inequality

$$
\lambda \tilde{p}^{k}=v_{k}^{\prime}\left(\omega^{k}+\tilde{z}^{k}\right)<v_{k}^{\prime}\left(\omega^{k}-\frac{\delta}{1-\varepsilon_{i}}\right) .
$$


As before we conclude, since $\tilde{p}^{k} \geq \varepsilon_{i-1}$

$$
\lambda \leq \max _{j} \frac{v_{j}^{\prime}\left(\omega^{j}-\frac{\delta}{1-\varepsilon_{i}}\right)}{\varepsilon_{i-1}} \stackrel{\text { def }}{=} K_{i} .
$$

From equation (96) we obtain then for all $j \in J^{c}$ that

$$
K_{i} \varepsilon_{i}>\lambda \tilde{p}^{j}=v_{j}^{\prime}\left(\omega^{j}+\tilde{z}^{j}\right)
$$

As $v_{j}$ satisfies the Inada conditions, we find that there is a value $\kappa_{i}>0$ such that if $0<\varepsilon_{i}<\kappa_{i}$, then $\tilde{z}^{j}>M$.

C.4 The homotopy argument. As a result of the previous subsection, we find that the price simplex $\Delta_{h}$ of every type $h$ contains a closed, compact, convex set $P_{h}$ such that $P_{h}$ has no point in common with the boundary of the simplex, and such that for every price vector

$$
p \in \Sigma_{h}=\Delta_{h} \backslash P_{h},
$$

the aggregate demand of at least one of the assets does not vanish.

Definition of the homotopy. We construct a homotopy of the heterogeneous problem to a homogeneous one, for which we actually know the equilibrium. This is done by introducing a homotopy parameter $\xi \in[0,1]$, and by introducing

$$
q_{h}^{s}(\xi)=\mathbb{E} p_{t+1}^{0}+\xi\left(p_{h t+1}^{0}-\mathbb{E} p_{t+1}^{0}\right)+y^{s} .
$$

Note that now all individual price simplexes and all individual demands depend on $\xi$. Moreover, for $\xi=0$ all agents are homogeneous in that they agree on the expected price (and on everything else), whereas the situation we are interested in is obtained for $\xi=1$.

We also define the sets

$$
\Delta(\xi)=\bigcap_{h} \Delta_{h}(\xi), \quad P(\xi)=\bigcap_{h} P_{h}(\xi), \quad \Sigma(\xi)=\Delta(\xi) \cap \bigcup_{h} \Sigma_{h}(\xi) .
$$

Note that $\Delta(\xi)$ and $P(\xi)$ are convex and compact, as they are the intersection of convex and compact sets. Moreover, note that the aggregate demand vector field is defined on the interior of $\Delta(\xi)$.

We shall show the following: for $\xi=0$, the system has a unique price equilibrium, and the index of the aggregate demand vector field on the boundary on $P(0)$ does not vanish. Since we know that the aggregate demand vector field does not vanish on $\Sigma(\xi)$ for any $\xi$, it follows that the index of the aggregate demand vector field on $P(1)$ is equal to 1 as well, and that therefore there exists a price equilibrium for the heterogeneous problem. It remains therefore to find a solution to the homogeneous problem and to determine its index. 
Solution to the homogeneous problem. If all agents are the same, the excess demand vector in equilibrium vanishes a priori and the first order conditions read as

$$
\begin{aligned}
u_{1}^{\prime}\left(w_{1}\right) & =\lambda p^{-1}, \\
u_{2}^{\prime}\left(w_{2}+\left(\mathbb{E} p_{t+1}^{0}+\bar{y}\right) \zeta^{0}\right)\left(\mathbb{E} p_{t+1}^{0}+\bar{y}\right) & =\lambda p^{0}, \\
u_{2}^{\prime}\left(w_{2}\right) \alpha_{j} & =\lambda p^{j}, \quad j=1, \cdots, n .
\end{aligned}
$$

These conditions can be solved for the equilibrium prices $p_{*}$ and the corresponding Lagrange multiplier $\lambda_{*}$; note that these prices are unique.

In order to compute the index of the aggregate demand vector field on the boundary of $P(0)$, it is sufficient to compute the local index of the unique equilibrium for $\xi=0$. For this, consider prices

$$
p^{j}(\varepsilon)=p_{*}^{j}+\varepsilon \bar{p}^{j} .
$$

As in subsection C.2, after a linear transformation we can as well consider maximizing the utility function

$$
\tilde{U}=\sum_{j=0}^{n+1} v_{j}\left(\omega_{j}+z^{j}\right)
$$

under the restriction $\sum_{j} p^{j}(\varepsilon) z^{j}=0$. Note that the local index of the equilibrium $p^{*}$ does not change under this linear transformation.

For every $\varepsilon$, we can maximize $\tilde{U}$ under the budget restriction to obtain the excess demands $z^{j}(\varepsilon)=\varepsilon \bar{z}^{j}+\mathrm{O}\left(\varepsilon^{2}\right)$ and the multiplier $\lambda(\varepsilon)=\lambda^{*}+\varepsilon \bar{\lambda}+\mathrm{O}\left(\varepsilon^{2}\right)$. The Lagrange equations read for $j=0, \cdots, n+1$ as

$$
v_{j}^{\prime}\left(\omega_{j}+z^{j}(\varepsilon)\right)=\lambda(\varepsilon) p^{j}(\varepsilon) .
$$

Taking derivatives left and right with respect to $\varepsilon$ and then setting $\varepsilon=0$ yields

$$
v_{j}^{\prime \prime}\left(\omega_{j}\right) \bar{z}^{j}=\lambda^{*} \bar{p}^{j}+\bar{\lambda} p_{*}^{j} .
$$

Doing the same with the budget restriction gives

$$
\sum_{j} p_{*}^{j} \bar{z}^{j}=0
$$

If we now multiply equation 97 with $\bar{z}^{j}$ and sum over $j$, using (98) we obtain

$$
\sum_{j} \bar{p}^{j} \bar{z}^{j}(\bar{p})=\frac{1}{\lambda^{*}} \sum_{j} v_{j}^{\prime \prime}\left(\omega_{j}\right)\left(\bar{z}^{j}\right)^{2}<0,
$$

since all $v_{j}$ 's have been assumed to be strictly concave. 
Setting $\bar{z}=\left(\bar{z}^{0}, \cdots, \bar{z}^{n+1}\right)$, we can now define a map $\psi: S^{n+2} \rightarrow S^{n+2}$ by

$$
\psi(\bar{p})=\frac{1}{\|\bar{z}(\bar{p})\|} \bar{z}(\bar{p})
$$

It is a consequence of equation (99) that this map is homotopic to the map $p \mapsto-p$ on $S^{n+2}$. But the degree of the latter map is \pm 1 . Then so is the degree of $\psi$; but then the index of the excess demand vector field is nonvanishing as well. Together with the remarks above, this completes the proof of the existence of a heterogeneous price equilibrium. 